\title{
A Baby Steps/Giant Steps Probabilistic Algorithm for Computing Roadmaps in Smooth Bounded Real Hypersurface
}

\author{
Mohab Safey el Din · Éric Schost
}

Received: 26 August 2009 / Revised: 19 December 2009 / Accepted: 19 December 2009 /

Published online: 5 February 2010

(C) Springer Science+Business Media, LLC 2010

\begin{abstract}
We consider the problem of constructing roadmaps of real algebraic sets. This problem was introduced by Canny to answer connectivity questions and solve motion planning problems. Given $s$ polynomial equations with rational coefficients, of degree $D$ in $n$ variables, Canny's algorithm has a Monte Carlo cost of $s^{n} \log (s) D^{O\left(n^{2}\right)}$ operations in $\mathbb{Q}$; a deterministic version runs in time $s^{n} \log (s) D^{O\left(n^{4}\right)}$. A subsequent improvement was due to Basu, Pollack, and Roy, with an algorithm of deterministic cost $s^{d+1} D^{O\left(n^{2}\right)}$ for the more general problem of computing roadmaps of a semi-algebraic set $(d \leq n$ is the dimension of an associated object).

We give a probabilistic algorithm of complexity $(n D)^{O\left(n^{1.5}\right)}$ for the problem of computing a roadmap of a closed and bounded hypersurface $V$ of degree $D$ in $n$ variables, with a finite number of singular points. Even under these extra assumptions, no previous algorithm featured a cost better than $D^{O\left(n^{2}\right)}$.
\end{abstract}

Keywords Computational real algebraic geometry $\cdot$ Algorithms · Roadmaps · Complexity

\section{Introduction}

Motivation Deciding connectivity properties in a semi-algebraic set $S$ is an important problem that appears in many fields, such as motion planning [35]. This general

\footnotetext{
M. Safey el Din ( $\varangle)$

SALSA Project, CNRS, UMR 7606, LIP6, Case 169, UPMC, Univ Paris 06, INRIA,

Paris-Rocquencourt Center, 4, Place Jussieu, 75252 Paris, France

e-mail: Mohab.Safey@lip6.fr

É. Schost

Computer Science Department, Room 415, Middlesex College, The University of Western Ontario,

London, Canada

e-mail: eschost@uwo.ca
} 
problem is reduced to computations in dimension 1, via the computation of a semialgebraic curve $\mathcal{R}$ that we call a roadmap. This curve should have a nonempty and connected intersection with each connected component of $S$ : then, connecting two points in $S$ is done by connecting these points to $\mathcal{R}$. Also, counting the connected components of $S$ is reduced to counting those of $\mathcal{R}$. Hence, a roadmap is used as the skeleton of connectivity decision routines for semi-algebraic sets. In addition to its direct interest, the computation of roadmaps is also used in more general algorithms allowing us to obtain semi-algebraic descriptions of the connected components of semi-algebraic sets [10, Chaps. 15-16]. Thus, improvements on the complexity of computing roadmaps impact the complexity of many fundamental procedures of effective real algebraic geometry.

Prior Results Let $\mathbf{Q}$ be a real field, and $\mathbf{R}$ be its real closure. The notion of a roadmap was introduced by Canny in [14, 15]; the resulting algorithm constructs a roadmap of a semi-algebraic set $S \subset \mathbf{R}^{n}$ but does not construct a path linking points of $S$. If $S$ is defined by $s$ equations and inequalities of degree bounded by $D$, the complexity is $s^{n} \log (s) D^{O\left(n^{4}\right)}$ arithmetic operations, and a Monte Carlo version of it runs in time $s^{n} \log (s) D^{O\left(n^{2}\right)}$ (to estimate running times, we always use arithmetic operations). Several subsequent works $[26,27]$ gave algorithms of $\operatorname{cost}(s D)^{n^{O(1)}}$; they culminate with the algorithm of Basu, Pollack, and Roy [8, 9] of cost $s^{d+1} D^{O\left(n^{2}\right)}$, where $d$ is the dimension of the algebraic set defined by all equations in the system. These algorithms reduce the general problem to the construction of a roadmap in a bounded and smooth hypersurface defined by a polynomial $f$ of degree $D$; the coefficients of $f$ lie in a field that contains several new infinitesimals.

Under the smoothness and compactness assumptions, and even in the simpler case of a polynomial $f$ with coefficients in $\mathbf{Q}$, none of the previous algorithms features a cost lower than $D^{O\left(n^{2}\right)}$, and none of them returns a roadmap of degree lower than $D^{O\left(n^{2}\right)}$. In this paper, we give the first known estimates of the form $(n D)^{O\left(n^{1.5}\right)}$ for this particular problem, in terms of output degree and running time.

All these previous works, and ours also, make use of computations of critical loci of projections and rely on geometric connectivity results for correctness. Before recalling the basics we need about algebraic sets and critical loci, we give precise definitions of roadmaps and state our main result.

Definitions and Main Result The original definition (found in [10]) is as follows. Let $S$ be a semi-algebraic set. A roadmap for $S$ (in the sense of [10]) is a semi-algebraic set $\mathcal{R}$ of dimension at most 1 which satisfies the following conditions:

$\mathrm{RM}_{1} \mathcal{R}$ is contained in $S$.

$\mathrm{RM}_{2}$ Each connected component of $S$ has a nonempty and connected intersection with $\mathcal{R}$.

$\mathrm{RM}_{3}$ For $x \in \mathbf{R}$, each connected component of $S_{x}$ intersects $\mathcal{R}$, where $S_{x}$ is the set of points of the form $\left(x, x_{2}, \ldots, x_{n}\right)$ in $S$.

We modify this definition (in particular by discarding $\mathrm{RM}_{3}$ ) for the following reasons. First, it is coordinate-dependent: if $\mathcal{R}$ is a roadmap of $S$, it is not necessarily true that $\phi(\mathcal{R})$ is a roadmap of $\phi(S)$ for a linear change of coordinates $\phi$. Besides, one interest 
of $\mathrm{RM}_{3}$ is to make it possible to connect two points in $S$ by adding additional curves to $\mathcal{R}$ : condition $\mathrm{RM}_{3}$ is well adjusted to the procedure given in [10], which we do not use here.

Hence, we propose a modification in the definition of roadmaps. We do not deal with semi-algebraic sets, but only with sets of the form $V \cap \mathbf{R}^{n}$, where $V \subset \mathbf{C}^{n}$ is an algebraic set, and $\mathbf{C}$ is the algebraic closure of $\mathbf{R}$. Our definition, like the previous one, allows us to count connected components and to construct paths between points in $V \cap \mathbf{R}^{n}$. Also, we generalize the definition to higher-dimensional "roadmaps," since our algorithm computes such objects. Thus, we say that an algebraic set $\mathcal{R} \subset \mathbf{C}^{n}$ is a roadmap of $V$ if:

$\mathrm{RM}_{1}^{\prime}$ Each semi-algebraically connected component of $V \cap \mathbf{R}^{n}$ has a nonempty and semi-algebraically connected intersection with $\mathcal{R} \cap \mathbf{R}^{n}$.

$\mathrm{RM}_{2}^{\prime}$ The set $\mathcal{R}$ is contained in $V$.

Remark that if $V$ is empty, $\mathcal{R}$ must be empty. If $V \cap \mathbf{R}^{n}$ is empty, then any algebraic set $\mathcal{R}$ contained in $V$ is a roadmap; if $V \cap \mathbf{R}^{n}$ is not empty, $\mathcal{R}$ is not empty. Next, we say that $\mathcal{R}$ is an $i$-roadmap of $V$ if in addition we have:

$\mathrm{RM}_{3}^{\prime}$ The set $\mathcal{R}$ is either $i$-equidimensional or empty.

Finally, it will be useful to add a finite set of control points $\mathcal{P}$ to our input, e.g., to test if the points of $\mathcal{P}$ are connected on $V \cap \mathbf{R}^{n}$. Then, $\mathcal{R}$ is a roadmap (resp. $i$-roadmap) of $(V, \mathcal{P})$ if we also have:

$\mathrm{RM}_{4}^{\prime}$ The set $\mathcal{R}$ contains $\mathcal{P} \cap V \cap \mathbf{R}^{n}$.

Using this modified definition, our main result is the following theorem. Hereafter, given a finite set $\mathcal{P}$, we write its cardinality $\delta_{\mathcal{P}}$ (but if $\mathcal{P}$ is empty, we take $\delta_{\mathcal{P}}=1$ ).

Theorem 1 Given $f$ squarefree in $\mathbf{Q}\left[X_{1}, \ldots, X_{n}\right]$ such that $V(f)$ has a finite number of singular points and $V(f) \cap \mathbf{R}^{n}$ is bounded, and given a set $\mathcal{P}$ of cardinality $\delta_{\mathcal{P}}$, one can compute a 1-roadmap of $(V(f), \mathcal{P})$ of degree $\delta_{\mathcal{P}}(n D)^{O\left(n^{1.5}\right)}$ in probabilistic time $\delta_{\mathcal{P}} O(1)(n D) O\left(n^{1.5}\right)$.

Computational Model and Probabilistic Aspects Our computational model is the algebraic RAM over $\mathbf{Q}$; we count at unit cost all operations $(+,-, \times)$, sign test, zero test, and inversion; thus, bit-complexity considerations are out of the scope of this paper. Note also that our set of operations is not enough to enable us to factor polynomials over $\mathbf{Q}$, which will occasionally induce extra complications.

Our algorithms are probabilistic, in the sense that they use random elements in $\mathbf{Q}$. The probabilistic aspects of our algorithm are twofold: first, we choose random changes of variables to ensure nice geometric properties. Second, we need to solve systems of polynomial equations; for our purpose, the algorithm with the best adapted cost (from [29], following [22-24]) is probabilistic as well (typically, it performs random combinations of the input system, etc.).

We have to make several random choices; every time a random element $\gamma$ is chosen in some parameter space $\mathbf{Q}^{i}$, there exists a nonzero polynomial $\Delta$ such that the choice is "lucky" as soon as $\Delta(\gamma) \neq 0$. If needed, one could estimate the degrees of 
the various polynomials $\Delta$ arising this way, though this is by no means straightforward.

Remark then that we can also deterministically compute a roadmap of $(V(f), \mathcal{P})$ of degree $\delta_{\mathcal{P}}(n D)^{O\left(n^{1.5}\right)}$ : the luckiness of our random choices can always be verified (as they essentially amount to check that some algebraic sets have an appropriate dimension); then, deterministic polynomial system solving algorithms replace the use of [29]. However, we lose the control on the complexity of the process.

Basic Definitions To describe our contribution, we need a few definitions; for standard notions not recalled here, see [19, 31, 36, 39]. An algebraic set $V \subset \mathbf{C}^{n}$ is the set of common zeros of some polynomial equations $f_{1}, \ldots, f_{s}$ in variables $X_{1}, \ldots, X_{n}$; we write $V=V\left(f_{1}, \ldots, f_{s}\right)$. The degree of an irreducible algebraic set $V \subset \mathbf{C}^{n}$ is the maximum number of intersection points between $V$ and a linear space of dimension $n-\operatorname{dim}(V)$; the degree of an arbitrary algebraic set is the sum of the degrees of its irreducible components.

The Zariski-tangent space to $V$ at $\mathbf{x} \in V$ is the vector space $T_{\mathbf{x}} V$ defined by the equations $\frac{\partial f}{\partial X_{1}}(\mathbf{x}) v_{1}+\cdots+\frac{\partial f}{\partial X_{n}}(\mathbf{x}) v_{n}=0$ for all polynomials $f$ that vanish on $V$.

We will only need to define regular and singular points for equidimensional algebraic sets. In this case, the regular points on $V$ are those points $\mathbf{x}$ where $\operatorname{dim}\left(T_{\mathbf{x}} V\right)=$ $\operatorname{dim}(V)$; the singular points are all other points. The set of regular (resp. singular) points is denoted by $\operatorname{reg}(V)$ (resp. $\operatorname{sing}(V)$ ). The set $\operatorname{sing}(V)$ is an algebraic subset of $V$ of smaller dimension than $V$.

Polar Varieties Canny's algorithm is the best known approach to computing roadmaps. Given an algebraic set $V$, it proceeds by computing some critical curves on $V$ and studying some distinguished points on these curves. One of our contributions is the use of higher-dimensional critical loci, called polar varieties, that were introduced by Todd [38] and used from the algorithmic point of view to compute sampling points in real algebraic sets in a series of papers by Bank, Giusti, Heintz, Pardo et al. [5-7]; our algorithms will rely on some key properties of polar varieties found in those references and [33]. For positive integers $i \leq n$, we denote by $\Pi_{i}$ the projection

$$
\begin{aligned}
\Pi_{i}: \mathbf{C}^{n} & \rightarrow \mathbf{C}^{i}, \\
\mathbf{x} & =\left(x_{1}, \ldots, x_{n}\right) \mapsto\left(x_{1}, \ldots, x_{i}\right) .
\end{aligned}
$$

Hereafter, we assume that $V$ is equidimensional. Then, the polar variety $w_{i}=$ $\operatorname{crit}\left(\Pi_{i}, \operatorname{reg}(V)\right)$ is the set of critical points of $\Pi_{i}$ on $\operatorname{reg}(V)$, that is, the set of all points $\mathbf{x} \in \operatorname{reg}(V)$ such that $\Pi_{i}\left(T_{\mathbf{x}} V\right) \neq \mathbf{C}^{i}$. The set $w_{i}$ may not be an algebraic set if $V$ has singular points; we will denote by $W_{i}$ its Zariski closure. It will also be useful to consider the $\operatorname{set} \operatorname{crit}\left(\Pi_{i}, V\right)=w_{i} \cup \operatorname{sing}(V)$; as it turns out, $\operatorname{crit}\left(\Pi_{i}, V\right)$ is an algebraic set, so it contains $W_{i}$. Assuming (as we will do) that $\operatorname{sing}(V)$ is finite, $\operatorname{crit}\left(\Pi_{i}, V\right)-W_{i}$ consists of at most a finite number of points, all in $\operatorname{sing}(V)$, or equivalently, $\operatorname{crit}\left(\Pi_{i}, V\right)=W_{i} \cup \operatorname{sing}(V)$.

If $V$ is given as $V\left(f_{1}, \ldots, f_{p}\right)$, is equidimensional of dimension $d=n-p$, and if the ideal $\left\langle f_{1}, \ldots, f_{p}\right\rangle$ is radical, then $\operatorname{crit}\left(\Pi_{i}, V\right)$ is the zero-set of $\left(f_{1}, \ldots, f_{p}\right)$ and 
of the $p$-minors taken from the Jacobian matrix of $\mathbf{f}=\left(f_{1}, \ldots, f_{p}\right)$ with respect to $\left(X_{i+1}, \ldots, X_{n}\right)$. Later on, the former matrix is written $\mathrm{jac}\left(\mathbf{f},\left[X_{i+1}, \ldots, X_{n}\right]\right)$, and its evaluation at a point $\mathbf{x} \in \mathbf{C}^{n}$ is written $\operatorname{jac}_{\mathbf{x}}\left(\mathbf{f},\left[X_{i+1}, \ldots, X_{n}\right]\right)$. The expected dimension of $W_{i}$, and of $\operatorname{crit}\left(\Pi_{i}, V\right)$ if $\operatorname{sing}(V)$ is finite, is $i-1$.

Using Polar Varieties Given $f$ of degree $D$ and $V=V(f)$, assuming that $V(f) \cap$ $\mathbf{R}^{n}$ is smooth and bounded, Canny's algorithm computes the critical curve $W_{2}$. Assuming $V(f) \cap \mathbf{R}^{n}$ bounded ensures that $W_{2}$ intersects each connected component of $V \cap \mathbf{R}^{n}$ but not that these intersections are connected. The solution consists in choosing a suitable family $\mathcal{C}^{\prime}=\left\{x_{1}, \ldots, x_{N}\right\} \subset \mathbf{R}$ so that the union of $W_{2}$ and $\mathcal{C}^{\prime \prime}=V \cap \Pi_{1}^{-1}\left(\mathcal{C}^{\prime}\right)$ is a roadmap of $V$ of dimension $n-2$.

To realize this, Canny's algorithm uses the following connectivity result: defining the (expectedly finitely many) points $\mathcal{C}=\operatorname{crit}\left(\Pi_{1}, V\right) \cup \operatorname{crit}\left(\Pi_{1}, W_{2}\right)$ and taking $\mathcal{C}^{\prime}=\Pi_{1}(\mathcal{C})$ gives an $(n-2)$-roadmap of $V$ of degree $D^{O(n)}$. Then, the algorithm recursively constructs a roadmap in $\mathcal{C}^{\prime \prime}=V \cap \Pi_{1}^{-1}\left(\mathcal{C}^{\prime}\right)$ following the same process; this is geometrically equivalent to a recursive call with input $f\left(x_{i}, X_{2}, \ldots, X_{n}\right)$ for all $x_{i} \in \mathrm{C}^{\prime}$. At each recursive call, the number of control points we compute is multiplied by $D^{O(n)}$, but the dimension of the input decreases by one only. Thus, the depth of the recursion is $n$, and the roadmap we get has degree $D^{O\left(n^{2}\right)}$.

Our algorithm relies on a new connectivity result that generalizes the one described above. We want to avoid the degree growth by performing recursive calls on inputs whose dimensions have decreased by $i \gg 1$. To this end, instead of considering the polar curve $W_{2}$ associated to a projection on a plane, we use polar varieties $W_{i}$ of higher dimension. As above, we have to consider suitable fibers $V \cap \Pi_{i-1}^{-1}(\mathbf{x})$ to repair the defaults of connectivity of $W_{i}$. To achieve this, we use the following new result (Theorem 14): define $\mathcal{C}=\operatorname{crit}\left(\Pi_{1}, V\right) \cup \operatorname{crit}\left(\Pi_{1}, W_{i}\right), \mathcal{C}^{\prime}=\Pi_{i-1}(\mathcal{C})$, and $\mathcal{C}^{\prime \prime}=V \cap \Pi_{i-1}^{-1}\left(\mathcal{C}^{\prime}\right)$; under some crucial (but technical) assumptions, $W_{i} \cup \mathcal{C}^{\prime \prime}$ is a roadmap of $V$ of dimension $\max (i-1, n-i)$. This leads to a more complex recursive algorithm; the optimal cut-off we could obtain that ensured all necessary assumptions has $i \simeq \sqrt{n}$.

Data Representation The output of our algorithms is a parameterization of an algebraic curve. If $V \subset \mathbf{C}^{e}$ is an algebraic curve defined over $\mathbf{Q}$, a one-dimensional parameterization of $V$ consists in polynomials $Q=\left(q, q_{0}, \ldots, q_{e}\right)$ in $\mathbf{Q}[U, T]$ and two linear forms $\tau=\tau_{1} X_{1}+\cdots+\tau_{e} X_{e}$ and $\eta=\eta_{1} X_{1}+\cdots+\eta_{e} X_{e}$ with coefficients in $\mathbf{Q}$, with $q$ squarefree, $\operatorname{gcd}\left(q, q_{0}\right)=1$, and such that $V$ is the Zariski closure of the set defined by

$$
q(\eta, \tau)=0, \quad X_{i}=\frac{q_{i}(\eta, \tau)}{q_{0}(\eta, \tau)} \quad(1 \leq i \leq e), q_{0}(\eta, \tau) \neq 0 .
$$

Given a parameterization $Q$, the corresponding curve $V$ is denoted by $Z(Q)$. The degree of $V$ is written $\delta_{Q}$; then, all polynomials in $Q$ can, and will, be taken of degree $\delta_{Q}^{O(1)}$, see [34].

Similarly, finite sets of points can be represented by means of univariate polynomials; then, a single linear form is needed, see, e.g., [1, 21-25, 32]. Concretely, to represent a finite subset $V$ of $\mathbf{C}^{e}$ defined over $\mathbf{Q}$, we use a linear form $\tau=$ 
$\tau_{1} X_{1}+\cdots+\tau_{e} X_{e}$ and polynomials $Q=\left(q, q_{1} \ldots, q_{e}\right)$ in $\mathbf{Q}[T]$, with $q$ squarefree, such that $V$ is given by

$$
q(\tau)=0, \quad X_{i}=q_{i}(\tau) \quad(1 \leq i \leq e)
$$

In this case, $\tau$ will be called a primitive element; $Q$ will be called a zero-dimensional parameterization. Again, $Z(Q) \subset \mathbf{C}^{e}$ will denote the finite set $V$, and $\delta_{Q}=|V|$ will be its cardinality (and all polynomials in $Q$ will have degree at most $\delta_{Q}$ ).

In both zero- and one-dimensional cases, if $Q$ represents a set of points $V$ in $\mathbf{C}^{e}$, with variables $X_{1}, \ldots, X_{e}$, it will be helpful to write $Q\left(X_{1}, \ldots, X_{e}\right)$ to indicate the variables used; $Q$ is defined over $\mathbf{Q}$ if all polynomials in it have coefficients in $\mathbf{Q}$. Finally, a parameterization of the empty set consists by convention of the unique polynomial $Q=(-1)$.

Using the Output Let us briefly sketch how to use the output of our algorithm to answer connectivity queries for points in a hypersurface $V=V(f)$. Given a set of control points $\mathcal{P}$ of cardinality 2 , the one-dimensional parameterization $Q=$ $\left(q, q_{0}, \ldots, q_{n}\right)$ we obtain from Theorem 1 only describes an open dense subset of a roadmap containing $\mathcal{P}$. It is possible to recover the finitely many missing points by means of a zero-dimensional parameterization $Q^{\prime}$ thereof, using Puiseux expansions at the points where both $q$ and $q_{0}$ vanish. Since all polynomials in $Q$ have degree $(n D)^{O\left(n^{1.5}\right)}$, this can be done in time $(n D)^{O\left(n^{1.5}\right)}$, using the algorithm of [18].

Given this, one can compute a Cylindrical Algebraic Decomposition adapted to the constructible sets defined by $Q$ and $Q^{\prime}$. In view of the simple shape of the defining polynomials, this takes time $(n D){ }^{O\left(n^{1.5}\right)}$ again. To compute adjacencies between cells, we use the algorithm of [35], which takes time $(n D)^{O\left(n^{1.5}\right)}$ using again the Puiseux expansion algorithm of [18].

Basic Notation The following conventions are used in the paper.

- $\mathbf{Q}$ is a real field, $\mathbf{R}$ is its real closure, and $\mathbf{C}$ is the algebraic closure of $\mathbf{R}$.

- If $X$ is a subset of either $\mathbf{C}^{n}$ or $\mathbf{R}^{n}$ and if $A$ is a subset of $\mathbf{R}$, we write $X_{A}=X \cap$ $\Pi_{1}^{-1}(A) \cap \mathbf{R}^{n}$. For $x$ in $\mathbf{R}$, we use the particular cases $X_{<x}=X_{]-\infty, x)}, X_{x}=X_{\{x\}}$, and $X_{\leq x}=X_{]-\infty, x]}$.

- A property is called generic (in a suitable parameter space) if it holds in a Zariskiopen dense subset of this parameter space.

- The closure notation $\bar{B}$ refers to the closure for the Euclidean topology.

- By convention, the empty set is considered finite.

- Finally, if $X \subset \mathbf{C}^{n}$ is the empty algebraic set, $\operatorname{crit}\left(\Pi_{i}, X\right)$ is formally defined as the empty set for all $i$.

\section{Global Properties of Roadmaps}

Consider an algebraic set $V \subset \mathbf{C}^{n}$ and a finite set of points $\mathcal{P}$ in $\mathbf{C}^{n}$. The following proposition will allow us to compute roadmaps of $(V, \mathcal{P})$ in a recursive manner. 
Proposition 2 Let $\mathcal{R}_{1}$ and $\mathcal{R}_{2}$ be algebraic sets such that $\mathcal{R}_{1} \cup \mathcal{R}_{2}$ is a roadmap of $(V, \mathcal{P})$ and such that $\mathcal{R}_{1} \cap \mathcal{R}_{2}$ is finite.

Let $\mathcal{R}_{1}^{\prime}$ and $\mathcal{R}_{2}^{\prime}$ be 1-roadmaps of respectively $\left(\mathcal{R}_{1},\left(\mathcal{R}_{1} \cap \mathcal{R}_{2}\right) \cup \mathcal{P}\right)$ and $\left(\mathcal{R}_{2},\left(\mathcal{R}_{1} \cap \mathcal{R}_{2}\right) \cup \mathcal{P}\right)$. Then $\mathcal{R}_{1}^{\prime} \cup \mathcal{R}_{2}^{\prime}$ is a 1-roadmap of $(V, \mathcal{P})$.

The proof of this proposition uses two lemmas.

Lemma 3 If $\mathcal{R}$ is a roadmap of $V$, then for each semi-algebraically connected component $C$ of $V \cap \mathbf{R}^{n}, C \cap \mathcal{R}$ is a semi-algebraically connected component of $\mathcal{R} \cap \mathbf{R}^{n}$.

Proof We know that $C \cap \mathcal{R}$ is semi-algebraically connected by $\mathrm{RM}_{1}^{\prime}$. Besides, $C$ is both open and closed in $V \cap \mathbf{R}^{n}$, so that $C \cap \mathcal{R}$ is open and closed in $\mathcal{R} \cap \mathbf{R}^{n}$.

Lemma 4 If $\mathcal{R}$ is a roadmap of $(V, \mathcal{P})$ and if $\mathcal{R}^{\prime}$ is a 1 -roadmap of $\mathcal{R}$ which contains $V \cap \mathcal{P} \cap \mathbf{R}^{n}$, then $\mathcal{R}^{\prime}$ is a 1-roadmap of $(V, \mathcal{P})$.

Proof The inclusions $\mathcal{R}^{\prime} \subset \mathcal{R} \subset V$ give $\mathrm{RM}_{2}^{\prime}$, and $\mathrm{RM}_{3}^{\prime}$ holds by assumption. Besides, since $\mathcal{R}^{\prime}$ contains $V \cap \mathcal{P} \cap \mathbf{R}^{n}$, we obtain $\mathrm{RM}_{4}^{\prime}$. Thus, we only miss $\mathrm{RM}_{1}^{\prime}$. We must prove that for each semi-algebraically connected component $C$ of $V \cap \mathbf{R}^{n}$, $C \cap \mathcal{R}^{\prime}$ is nonempty and semi-algebraically connected. Since $\mathcal{R}$ is a roadmap of $V, C \cap \mathcal{R}$ is a semi-algebraically connected component of $\mathcal{R} \cap \mathbf{R}^{n}$ (Lemma 3). For the same reason, since $\mathcal{R}^{\prime}$ is a roadmap of $\mathcal{R}, C \cap \mathcal{R} \cap \mathcal{R}^{\prime}=C \cap \mathcal{R}^{\prime}$ is a semialgebraically connected component of $\mathcal{R}^{\prime} \cap \mathbf{R}^{n}$.

We can now prove the proposition. We first prove that $\mathcal{R}_{1}^{\prime} \cup \mathcal{R}_{2}^{\prime}$ contains $V \cap$ $\mathcal{P} \cap \mathbf{R}^{n}$. By assumption, $\mathcal{R}_{1}^{\prime}$ and $\mathcal{R}_{2}^{\prime}$ contain respectively $\mathcal{R}_{1} \cap \mathcal{P} \cap \mathbf{R}^{n}$ and $\mathcal{R}_{2} \cap \mathcal{P} \cap$ $\mathbf{R}^{n}$. Since $\mathcal{R}_{1} \cup \mathcal{R}_{2}$ is a roadmap of $(V, \mathcal{P})$, we have by definition that $V \cap \mathscr{P} \cap \mathbf{R}^{n} \subset$ $\left(\mathcal{R}_{1} \cup \mathcal{R}_{2}\right) \cap \mathbf{R}^{n}$, and thus $V \cap \mathscr{P} \cap \mathbf{R}^{n} \subset\left(\mathcal{R}_{1} \cup \mathcal{R}_{2}\right) \cap \mathcal{P} \cap \mathbf{R}^{n}$; this is contained in $\mathcal{R}_{1}^{\prime} \cup \mathcal{R}_{2}^{\prime}$ by the former remark.

Besides, $\mathcal{R}_{1}^{\prime} \cup \mathcal{R}_{2}^{\prime}$ is either empty or 1-equidimensional. As a consequence, in view of Lemma 4, it is sufficient to prove that $\mathcal{R}_{1}^{\prime} \cup \mathcal{R}_{2}^{\prime}$ is a roadmap of $\mathcal{R}_{1} \cup \mathcal{R}_{2}$.

If $\left(\mathcal{R}_{1} \cup \mathcal{R}_{2}\right) \cap \mathbf{R}^{n}$ is empty, we are done. Else, let $C$ be a semi-algebraically connected component of $\left(\mathcal{R}_{1} \cup \mathcal{R}_{2}\right) \cap \mathbf{R}^{n}$. First, we prove that $C \cap\left(\mathcal{R}_{1}^{\prime} \cup \mathcal{R}_{2}^{\prime}\right)$ is not empty. Indeed, $C$ contains a semi-algebraically connected component of either $\mathcal{R}_{1} \cap \mathbf{R}^{n}$ or $\mathcal{R}_{2} \cap \mathbf{R}^{n}$ (since it contains a point of say $\mathcal{R}_{1}$, it contains its semialgebraically connected component); and as such, $C$ intersects either $\mathcal{R}_{1}^{\prime}$ or $\mathcal{R}_{2}^{\prime}$.

We prove now that $C \cap\left(\mathcal{R}_{1}^{\prime} \cup \mathcal{R}_{2}^{\prime}\right)$ is semi-algebraically connected. Consider a pair of points $\mathbf{x}, \mathbf{x}^{\prime}$ in $C \cap\left(\mathcal{R}_{1}^{\prime} \cup \mathcal{R}_{2}^{\prime}\right)$. Since $C$ is semi-algebraically connected, there exists a continuous path $\gamma:[0,1] \rightarrow C$ such that $\gamma(0)=\mathbf{x}$ and $\gamma(1)=\mathbf{x}^{\prime}$. Since $\mathcal{R}_{1} \cap \mathcal{R}_{2}$ is finite, we can reparameterize $\gamma$, to ensure that $\gamma^{-1}\left(\mathcal{R}_{1} \cap \mathcal{R}_{2}\right)$ is finite. Denote by $t_{1}<\cdots<t_{r}$ the set $\gamma^{-1}\left(\mathcal{R}_{1} \cap \mathcal{R}_{2}\right)$ and let $t_{0}=0$ and $t_{r+1}=1$. Then, we replace $\gamma$ by a semi-algebraic continuous path $\gamma^{\prime}$ defined on the segments $\left[t_{i}, t_{i+1}\right]$ as follows:

- For $1 \leq i<r, \gamma\left(\left(t_{i}, t_{i+1}\right)\right)$ is semi-algebraically connected and contained in $\mathcal{R}_{1} \cup$ $\mathcal{R}_{2}-\mathcal{R}_{1} \cap \mathcal{R}_{2}$; because both $\mathcal{R}_{1}$ and $\mathcal{R}_{2}$ are closed, $\gamma\left(\left(t_{i}, t_{i+1}\right)\right)$ is contained in (say) $\mathcal{R}_{1}$. By continuity, $\gamma\left(\left[t_{i}, t_{i+1}\right]\right)$ is contained in $\mathcal{R}_{1}$ and thus actually in a semi-algebraically connected component $C^{\prime}$ of $\mathcal{R}_{1} \cap \mathbf{R}^{n}$. 
Note first that both $\gamma\left(t_{i}\right)$ and $\gamma\left(t_{i+1}\right)$ are in $\mathcal{R}_{1} \cap \mathcal{R}_{2}$ and thus in $\mathcal{R}_{1}^{\prime}$. Besides, since $\mathcal{R}_{1}^{\prime}$ is a roadmap of $\mathcal{R}_{1}, C^{\prime} \cap \mathcal{R}_{1}^{\prime}$ is semi-algebraically connected, so there exists a continuous semi-algebraic path $\gamma^{\prime}:\left[t_{i}, t_{i+1}\right] \rightarrow C^{\prime} \cap \mathcal{R}_{1}^{\prime}$ with $\gamma^{\prime}\left(t_{i}\right)=$ $\gamma\left(t_{i}\right)$ and $\gamma^{\prime}\left(t_{i+1}\right)=\gamma\left(t_{i+1}\right)$.

Now, because $C^{\prime}$ is a semi-algebraically connected component of $\mathcal{R}_{1} \cap \mathbf{R}^{n}$ and $C$ is a semi-algebraically connected component of $\left(\mathcal{R}_{1} \cup \mathcal{R}_{2}\right) \cap \mathbf{R}^{n}$, we deduce $C^{\prime} \subset C$, so the image of $\gamma^{\prime}$ is in $C \cap \mathcal{R}_{1}^{\prime}$ and thus in $C \cap\left(\mathcal{R}_{1}^{\prime} \cup \mathcal{R}_{2}^{\prime}\right)$.

- The case $i=0$ needs to be taken care of only if $t_{0}<t_{1}$, so that $\mathbf{x}=\gamma\left(t_{0}\right)$ is either in $\mathcal{R}_{1}$ or in $\mathcal{R}_{2}$, but not in both. As before, we start by remarking that $\gamma\left(\left[t_{0}, t_{1}\right]\right)$ is contained in a semi-algebraically connected component $C^{\prime}$ of say $\mathcal{R}_{1} \cap \mathbf{R}^{n}$, with $C^{\prime} \subset C$. This implies that $\mathbf{x}=\gamma\left(t_{0}\right)$ is in $\mathcal{R}_{1}$; since $\mathbf{x}$ is in $\mathcal{R}_{1}^{\prime} \cup \mathcal{R}_{2}^{\prime}$, it is actually in $\mathcal{R}_{1}^{\prime}$ (because it cannot be in $\mathcal{R}_{2}^{\prime}$, since then it would be in $\mathcal{R}_{2}$ ). As before, $\gamma\left(t_{1}\right)$ is in $\mathcal{R}_{1}^{\prime}$, and the conclusion follows as in the previous case. The case $i=r$ is dealt with similarly.

\section{Two Auxiliary Results}

This section proves two results that will be used toward the proof of our main connectivity theorem. We consider an equidimensional algebraic set $Z \subset \mathbf{C}^{n}$ of dimension $d>0$ and study various connectivity properties of sets of the form $Z_{<x}$ or $Z_{\leq x}$.

\subsection{First Result}

For $x \in \mathbf{R}$, we are interested here in the properties of the semi-algebraically connected components of $Z_{<x}$ in the neighborhood of the hyperplane $\Pi_{1}^{-1}(x)$.

Proposition 5 Let $x$ be in $\mathbf{R}$, and let $\gamma: A \rightarrow Z_{\leq x}-Z_{x} \cap \operatorname{crit}\left(\Pi_{1}, Z\right)$ be a continuous semi-algebraic map, where $A \subset \mathbf{R}^{k}$ is a semi-algebraically connected semi-algebraic set. Then there exists a unique semi-algebraically connected component $B$ of $Z_{<x}$ such that $\gamma(A) \subset \bar{B}$.

This subsection is devoted to prove this proposition using a series of lemmas; some of them are elementary. The first lemma is a direct consequence of the semi-algebraic implicit function theorem [10, Theorem 3.25].

Lemma 6 Let $\mathbf{x}=\left(x_{1}, \ldots, x_{n}\right)$ be in $Z \cap \mathbf{R}^{n}-\operatorname{crit}\left(\Pi_{1}, Z\right)$. Then, there exists a permutation $\sigma$ of $\{1, \ldots, n\}$ that fixes 1 , such that the following holds. Let $\mathbf{x}^{\prime}=$ $\left(x_{\sigma(\ell)}, \ell \leq d\right) \in \mathbf{R}^{d}$. There exist open Euclidean neighborhoods $N^{\prime} \subset \mathbf{R}^{d}$ of $\mathbf{x}^{\prime}$ and $N \subset \mathbf{R}^{n}$ of $\sigma(\mathbf{x})$, and continuous semi-algebraic functions $\mathbf{f}=\left(f_{1}, \ldots, f_{n-d}\right)$ defined on $N^{\prime}$ such that we have

$$
\sigma(Z) \cap N=\left\{\left(\mathbf{y}^{\prime}, \mathbf{f}\left(\mathbf{y}^{\prime}\right)\right) \mid \mathbf{y}^{\prime} \in N^{\prime}\right\}
$$

As a consequence, we obtain the following result, similar to Proposition 7.3 in [10]. 
Lemma 7 Let $\mathbf{x}=\left(x_{1}, \ldots, x_{n}\right)$ be in $Z \cap \mathbf{R}^{n}-\operatorname{crit}\left(\Pi_{1}, Z\right)$. There exists an open semi-algebraically connected neighborhood $X_{\mathbf{x}}$ of $\mathbf{x}$ such that $\left(Z \cap X_{\mathbf{x}}\right)_{<x_{1}}$ is nonempty and semi-algebraically connected and such that $\left(Z \cap X_{\mathbf{x}}\right)_{x_{1}}$ is contained in $\overline{\left(Z \cap X_{\mathbf{x}}\right)_{<x_{1}}}$.

Proof Without loss of generality, let us assume that $\mathbf{x}=\mathbf{0}$, and let $\sigma, N^{\prime}, N$, and $\mathbf{f}$ be obtained by applying Lemma 6; we let $\mathbf{F}$ be the mapping $\mathbf{y}^{\prime} \in N^{\prime} \mapsto\left(\mathbf{y}^{\prime}, \mathbf{f}\left(\mathbf{y}^{\prime}\right)\right) \in N$.

Let $\eta_{0}>0$ be such that the closed ball $\overline{\mathcal{B}\left(\mathbf{0}, \eta_{0}\right)}$ is contained in $N^{\prime}$, and let $K \geq 1$ be such that for all $\mathbf{y}^{\prime}$ in $\mathscr{B}\left(\mathbf{0}, \eta_{0}\right)$, we have the inequality

$$
\left\|\mathbf{F}\left(\mathbf{y}^{\prime}\right)\right\|_{\mathbf{R}^{n}} \leq K\left\|\mathbf{y}^{\prime}\right\|_{\mathbf{R}^{d}},
$$

where all norms are 2-norms; for $K$, we can take the maximum of $\|d \mathbf{F}\|$ on $\overline{\mathscr{B}\left(\mathbf{0}, \eta_{0}\right)}$, by Proposition 2.9.6 in [12]. Let finally $\varepsilon_{0}>0$ be such that the open ball $\mathscr{B}\left(\mathbf{0}, \varepsilon_{0}\right) \subset$ $\mathbf{R}^{n}$ is contained in $N$. We define $\varepsilon=\min \left(\eta_{0}, \varepsilon_{0} / K\right)$ and

$$
X^{\prime}=\mathscr{B}(\mathbf{0}, \varepsilon) \subset \mathbf{R}^{d} \quad \text { and } \quad X=\mathscr{B}(\mathbf{0}, K \varepsilon) \cap\left(X^{\prime} \times \mathbf{R}^{n-d}\right) \subset \mathbf{R}^{n},
$$

where both $\mathscr{B}(\mathbf{0},$.$) denote open balls. We proceed to prove that taking X_{\mathbf{x}}=X$ satisfies the claims of the proposition. First, $X$ is open, semi-algebraic, and semialgebraically connected (because it is the intersection of two convex sets).

Next, we prove that $X \cap \sigma(Z)=\mathbf{F}\left(X^{\prime}\right)$. Note that $X$ is contained in $\mathcal{B}(\mathbf{0}, K \varepsilon)$, thus in $\mathcal{B}\left(\mathbf{0}, \varepsilon_{0}\right)$, and thus in $N$. We deduce from Lemma 6

$$
\sigma(Z) \cap X=\sigma(Z) \cap N \cap X=\mathbf{F}\left(N^{\prime}\right) \cap X .
$$

Hence, it suffices to prove that $\mathbf{F}\left(N^{\prime}\right) \cap X=\mathbf{F}\left(X^{\prime}\right)$. Let first $\mathbf{y}=\mathbf{F}\left(\mathbf{y}^{\prime}\right)$ be a point in $\mathbf{F}\left(N^{\prime}\right) \cap X$. Since $\mathbf{y}$ is in $X$, it is in $X^{\prime} \times \mathbf{R}^{n-d}$; because $\mathbf{F}\left(\mathbf{y}^{\prime}\right)=\left(\mathbf{y}^{\prime}, \mathbf{f}\left(\mathbf{y}^{\prime}\right)\right)$, this means that $\mathbf{y}^{\prime}$ is in $X^{\prime}$. Conversely, let $\mathbf{y}^{\prime}$ be in $X^{\prime}$. Then $\mathbf{y}=\mathbf{F}\left(\mathbf{y}^{\prime}\right)=\left(\mathbf{y}^{\prime}, \mathbf{f}\left(\mathbf{y}^{\prime}\right)\right)$ is in $X^{\prime} \times \mathbf{R}^{n-d}$. Also, because $\mathbf{y}^{\prime}$ is in $\mathscr{B}(\mathbf{0}, \varepsilon)$ and thus in $\mathscr{B}\left(\mathbf{0}, \eta_{0}\right)$, we have $\|\mathbf{y}\|_{\mathbf{R}^{n}} \leq$ $K\left\|\mathbf{y}^{\prime}\right\|_{\mathbf{R}^{d}} \leq K \varepsilon$. Hence, $\mathbf{y}$ is in $B(\mathbf{0}, K \varepsilon)$ and thus in $X$. So our claim is established.

Since $\sigma(Z) \cap X=\mathbf{F}\left(X^{\prime}\right)$, we deduce that $(\sigma(Z) \cap X)_{<x_{1}}=\mathbf{F}\left(X^{\prime}\right)_{<x_{1}}=\mathbf{F}\left(X^{\prime}<x_{1}\right)$. Since $X^{\prime}{ }_{<x_{1}}$ is nonempty and semi-algebraically connected and $\mathbf{F}$ is semi-algebraic continuous, its image $(\sigma(Z) \cap X)_{<x_{1}}$ is nonempty and semi-algebraically connected. Since $\sigma$ leaves the first coordinate invariant, this is thus also the case for $(Z \cap X)_{<x_{1}}$, as claimed.

For the last claim, remark that $(\sigma(Z) \cap X)_{x_{1}}=\mathbf{F}\left(X^{\prime}\right)_{x_{1}}=\mathbf{F}\left(X^{\prime} x_{1}\right)$. Since $X^{\prime}{ }_{x_{1}}$ is contained in $\overline{X^{\prime}<x_{1}}$, we deduce that $(\sigma(Z) \cap X)_{x_{1}}$ is contained in $\mathbf{F}\left(\overline{X^{\prime}<x_{1}}\right)$. Since $\overline{X_{<x_{1}}^{\prime}}$ is bounded and closed and $\mathbf{F}$ is continuous, $\mathbf{F}\left(\overline{X^{\prime}<x_{1}}\right)$ is bounded and closed too, by Theorem 3.20 in [10]. Because $\mathbf{F}$ is continuous, we also have

$$
\mathbf{F}\left(X^{\prime} x_{1}\right) \subset \mathbf{F}\left(\overline{X_{<x_{1}}^{\prime}}\right) \subset \overline{\mathbf{F}\left(X^{\prime}<x_{1}\right)},
$$

from which we deduce that

$$
\mathbf{F}\left(\overline{X^{\prime}<x_{1}}\right)=\overline{\mathbf{F}\left(X^{\prime}<x_{1}\right)} \text {. }
$$

This shows that $(\sigma(Z) \cap X)_{x_{1}}$ is contained in $\overline{\mathbf{F}\left(X^{\prime}<x_{1}\right)}$, which equals $\overline{(\sigma(Z) \cap X)_{<x_{1}}}$ by the previous paragraph. Up to restoring the initial order on the variables, this establishes our last claim. 
Lemma 8 Let $\mathbf{x}=\left(x_{1}, \ldots, x_{n}\right)$ be in $Z \cap \mathbf{R}^{n}-\operatorname{crit}\left(\Pi_{1}, Z\right)$. There exists a unique semi-algebraically connected component $B_{\mathbf{x}}$ of $Z_{<x_{1}}$ such that $\left(Z \cap X_{\mathbf{x}}\right)_{<x_{1}} \subset B_{\mathbf{x}}$, where $X_{\mathbf{x}}$ is defined in Lemma 7. Besides, $B_{\mathbf{X}}$ is the unique semi-algebraically connected component of $Z_{<x_{1}}$ such that $\mathbf{x}$ is in $\overline{B_{\mathbf{x}}}$.

Proof Because $\left(Z \cap X_{\mathbf{x}}\right)_{<x_{1}}$ is nonempty and semi-algebraically connected (Lemma 7), it is contained in a semi-algebraically connected component $B_{\mathbf{x}}$ of $Z_{<x_{1}}$. The semi-algebraically connected components of $Z_{<x_{1}}$ are pairwise disjoint, so $B_{\mathbf{x}}$ is well defined. By Lemma 7 again, $\mathbf{x}$ is in $\overline{\left(Z \cap X_{\mathbf{x}}\right)_{<x_{1}}}$ and thus in $\overline{B_{\mathbf{x}}}$. Suppose finally that $\mathbf{x}$ is in $\overline{B^{\prime}}$ for another semi-algebraically connected component $B^{\prime}$ of $Z_{<x_{1}}$. Then, there exists a point of $B^{\prime}$ in $X_{\mathbf{x}}$, because $X_{\mathbf{x}}$ is open. This point is in $\left(Z \cap X_{\mathbf{x}}\right)_{<x_{1}}$ and thus in $B_{\mathbf{X}}$ as well, which yields a contradiction.

Lemma 9 Let $\mathbf{x}=\left(x_{1}, \ldots, x_{n}\right)$ be in $Z \cap \mathbf{R}^{n}-\operatorname{crit}\left(\Pi_{1}, Z\right)$. For $\mathbf{x}^{\prime}$ in $\left(Z \cap X_{\mathbf{x}}\right)_{x_{1}}-$ $\operatorname{crit}\left(\Pi_{1}, Z\right)$, we have $B_{\mathbf{x}^{\prime}}=B_{\mathbf{x}}$.

Proof We know that $\mathbf{x}^{\prime}$ is in $\overline{B_{\mathbf{x}^{\prime}}}$. Since $\mathbf{x}^{\prime}$ is in $X_{\mathbf{X}}$ and $X_{\mathbf{X}}$ is open, there exists a point of $B_{\mathbf{x}^{\prime}}$ in $\left(Z \cap X_{\mathbf{x}}\right)_{<x_{1}}$. This point is in $B_{\mathbf{x}}$ as well, so $B_{\mathbf{x}^{\prime}}=B_{\mathbf{x}}$.

Lemma 10 Let $x$ be in $\mathbf{R}$, and let $\gamma$ be a continuous semi-algebraic map $A \rightarrow Z_{x}-$ $\operatorname{crit}\left(\Pi_{1}, Z\right)$, where $A \subset \mathbf{R}^{k}$ is a semi-algebraically connected set. Then, there exists a unique semi-algebraically connected component $B$ of $Z_{<x}$ such that for all $\mathbf{a} \in A$, $\gamma(\mathbf{a}) \in \bar{B}$.

Proof By Lemma 9, the map $\mathbf{a} \mapsto B_{\gamma}$ (a) is locally constant, so it is constant, since $A$ is semi-algebraically connected. So, with $B=B_{\gamma\left(\mathbf{a}_{0}\right)}$, for some $\mathbf{a}_{0}$ in $A$, we have $B_{\gamma(\mathbf{a})}=B$ for all $\mathbf{a}$ in $A$, and thus $\gamma(\mathbf{a}) \in \bar{B}$ for all $\mathbf{a} \in A$ by Lemma 8 . The uniqueness is a consequence of the second part of Lemma 8 .

We can now prove Proposition 5. Let $\gamma$ be a continuous semi-algebraic map $A \rightarrow$ $Z_{\leq x}-Z_{x} \cap \operatorname{crit}\left(\Pi_{1}, Z\right)$, where $A \subset \mathbf{R}^{k}$ is a connected semi-algebraic set; we prove that $\gamma(A)$ is contained in the closure $\bar{B}$ of a semi-algebraically connected component $B$ of $Z_{<x}$.

If $\gamma(A)$ is contained in $Z_{<x}$, then, since it is semi-algebraically connected, it is contained in a uniquely defined semi-algebraically connected component $B$ of $Z_{<x}$, and we are done.

Else, let $G=\gamma^{-1}\left(Z_{x}\right)$, which is closed in $A$. We decompose it into its semialgebraically connected components $G_{1}, \ldots, G_{N}$. Because all $G_{i}$ are closed in $G$, they are closed in $A$. Let also $H_{1}, \ldots, H_{M}$ be the semi-algebraically connected components of $A-G$; hence, the $H_{j}$ are open in $A$ (because they are open in $A-G$, which is open in $A$ ). The sets $G_{i}$ and $H_{j}$ form a partition of $A$; we assign to each of them a semi-algebraically connected component of $Z_{<x}$.

- Since $G_{i}$ is semi-algebraically connected and $\gamma\left(G_{i}\right)$ is contained in $Z_{x}-$ $\operatorname{crit}\left(\Pi_{1}, Z\right)$, Lemma 10 shows that there exists a unique semi-algebraically connected component $B_{G_{i}}$ of $Z_{<x}$ such that $\gamma\left(G_{i}\right) \subset \overline{B_{G_{i}}}$. 
- Since $H_{j}$ is semi-algebraically connected and $\gamma\left(H_{j}\right)$ is contained in $Z_{<x}$, there exists a unique semi-algebraically connected component $B_{H_{j}}$ of $Z_{<x}$ that contains $\gamma\left(H_{j}\right)$. Since $\gamma$ is continuous, we still have $\gamma\left(\overline{H_{j}}\right) \subset \overline{B_{H_{j}}}$.

Since the sets $G_{i}$ and $H_{j}$ form a partition of $A$, we deduce from the previous construction a function $\mathbf{a} \mapsto B_{\mathbf{a}}$ in the obvious manner: if $\mathbf{a}$ is in $G_{i}$, we let $B_{\mathbf{a}}=B_{G_{i}}$; if $\mathbf{a}$ is in $H_{j}$, we let $B_{\mathbf{a}}=B_{H_{j}}$. It remains to prove that this function is constant on $A$; then, if we let $B$ be the common value $B_{\mathbf{a}}$ for all $\mathbf{a}$ in $A, \gamma(\mathbf{a})$ is in $\bar{B}$ by construction (uniqueness is clear). To do so, it is sufficient to prove that for any $\mathbf{a}$ in $A$, there exists a neighborhood $N_{\mathbf{a}}$ of a such that for all $\mathbf{a}^{\prime}$ in $N_{\mathbf{a}}, B_{\mathbf{a}}=B_{\mathbf{a}^{\prime}}$.

- If $\mathbf{a}$ is in some $H_{j}$, we are done, since $H_{j}$ is open, and $\mathbf{a} \mapsto B_{\mathbf{a}}$ is constant on $H_{j}$.

- Else, $\mathbf{a}$ is in some $G_{i}$. Remark that $\mathbf{a}$ is in the closure of no other $G_{i^{\prime}}$, since the $G_{i}$ are closed; however, a can belong to the closure of some $H_{j}$. Let $J$ be the set of indices such that $\mathbf{a}$ is in $\overline{H_{j}}$ for $j$ in $J$, and let $e>0$ be such that the open ball $\mathscr{B}(\mathbf{a}, e)$ centered at $\mathbf{a}$ and of radius $e$ intersects no $G_{i^{\prime}}$ for $i^{\prime} \neq i$ and no $\overline{H_{j}}$ for $j$ not in $J$. Since a is in $G_{i}$, we know that $\gamma(\mathbf{a})$ is in $\overline{B_{G_{i}}}$; for $j$ in $J$, since $\mathbf{a}$ is in $\overline{H_{j}}$, we also have that $\gamma(\mathbf{a})$ is in $\overline{B_{H_{j}}}$. However, since $\gamma(\mathbf{a})$ is in $Z_{x}-\operatorname{crit}\left(\Pi_{1}, Z\right)$, the second statement in Lemma 8 implies that $B_{G_{i}}=B_{H_{j}}$. Since every $\mathbf{a}^{\prime}$ in $\mathscr{B}(\mathbf{a}, e)$ is either in $G_{i}$ or in some $H_{j}$ with $j$ in $J$, we are done.

This concludes the proof of Proposition 5. The following corollary will be of use.

Corollary 11 Let $x \in \mathbf{R}$ be such that $Z_{x} \cap \operatorname{crit}\left(\Pi_{1}, Z\right)=\emptyset$, and let $C$ be a semialgebraically connected component of $Z_{\leq x}$. Then if $C_{<x}$ is nonempty, it is semialgebraically connected.

Proof Consider the inclusion map $C \rightarrow Z_{\leq x}$. Since $Z_{x} \cap \operatorname{crit}\left(\Pi_{1}, Z\right)$ is empty, this map satisfies the assumptions of Proposition 5; this implies that there exists a unique semi-algebraically connected component $B$ of $Z_{<x}$ such that $C \subset \bar{B}$. This equality implies that $C_{<x}$ is contained in $\bar{B}_{<x}$; one easily checks that $B=\bar{B}_{<x}$, so that $C_{<x} \subset B$.

If $C_{<x}$ is not empty, let $B^{\prime}$ be a semi-algebraically connected component of $C_{<x}$, so that $B^{\prime}$ is actually a semi-algebraically connected component of $Z_{<x}$. The inclusion $B^{\prime} \subset C_{<x}$ implies that $B^{\prime} \subset C_{<x} \subset B$ and thus $B^{\prime}=C_{<x}=B$. Since $B$ is semialgebraically connected, $C_{<x}$ is semi-algebraically connected too, as claimed.

\subsection{Second Result}

The following statement is in the vein of Morse's Lemma A [10, Theorem 7.5]. Proofs of Morse's lemma (and of similar statements) use the Ehresmann fibration theorem [13, Theorem 3.4], which relies on the integration of vector fields and thus requires the base fields to be $\mathbb{R}$ or $\mathbb{C}$. Here, we keep on working with base fields $\mathbf{R}$ and $\mathbf{C}$ by considering closed and bounded semi-algebraic sets of $\mathbf{R}^{n}$, which share a lot of properties with compact semi-algebraic sets of $\mathbb{R}^{n}$. As to the notion of differentiability, we will use $\mathcal{C}^{\infty}$ semi-algebraic functions, also known as Nash functions. With this in mind, we will be able to rely on a Nash version of the Ehresmann fibration theorem [16, Theorems 2.4 and 3.1]. 
Proposition 12 Let $A \subset(-\infty, w) \times \mathbf{R}^{n-1}$ be a semi-algebraically connected, bounded, semi-algebraic set, and let $v \in \mathbf{R}$ be such that $v<w$, such that $A_{(v, w)}$ is a nonempty Nash manifold, closed in $(v, w) \times \mathbf{R}^{n-1}$, and such that $\Pi_{1}$ is a submersion on $A_{(v, w)}$. Then, for all $x$ in $[v, w), A_{\leq x}$ is nonempty and semi-algebraically connected.

Proof Let us first check that $\Pi_{1}: A_{(v, w)} \rightarrow(v, w)$ is a semi-algebraically "proper" mapping in the sense that the preimage of a closed and bounded set is closed and bounded.

Let $K$ be a closed and bounded set in $(v, w)$. Since $A$ is bounded, its preimage is bounded. To prove that $A_{(v, w)} \cap \Pi_{1}^{-1}(K)=A_{(v, w)} \cap\left(K \times \mathbf{R}^{n-1}\right)$ is closed in $\mathbf{R}^{n}$, recall that by assumption, there exists a closed set $X \subset \mathbf{R}^{n}$ such that $A_{(v, w)}=$ $X \cap\left((v, w) \times \mathbf{R}^{n-1}\right)$; in the next paragraph, it will be convenient to take $X$ bounded (this is allowed, since $A$ is). Then, $A_{(v, w)} \cap\left(K \times \mathbf{R}^{n-1}\right)=X \cap\left(K \times \mathbf{R}^{n-1}\right)$, which is closed in $\mathbf{R}^{n}$.

Next, we prove that $\Pi_{1}\left(A_{(v, w)}\right)=(v, w)$. Remark first that the image $\Pi_{1}\left(A_{(v, w)}\right)$ is open in $(v, w)$, since $\Pi_{1}$ is a submersion on $A_{(v, w)}$. Besides, with $X$ as before, we have $\Pi_{1}\left(A_{(v, w)}\right)=\Pi_{1}\left(X \cap\left((v, w) \times \mathbf{R}^{n-1}\right)\right)=\Pi_{1}(X) \cap(v, w)$. Since $X$ is closed and bounded, $\Pi_{1}(X)$ is closed. This implies that $\Pi_{1}\left(A_{(v, w)}\right)$ is closed in $(v, w)$ and finally that $\Pi_{1}\left(A_{(v, w)}\right)=(v, w)$.

Let $\zeta$ be fixed in $(v, w)$. The previous paragraph shows that we can apply the Nash version of the Ehresmann fibration theorem [16, Theorems 2.4(iii) ${ }^{\prime}$ and 3.1] to the projection $\Pi_{1}$. This gives us a Nash diffeomorphism of the form

$$
\begin{gathered}
\Psi: A_{(v, w)} \rightarrow(v, w) \times A_{\zeta}^{\prime}, \\
(\alpha, \mathbf{a}) \mapsto(\alpha, \psi(\alpha, \mathbf{a})),
\end{gathered}
$$

where $A_{\zeta}^{\prime} \subset \mathbf{R}^{n-1}$ is the set $\left\{\left(x_{2}, \ldots, x_{n}\right) \mid\left(\zeta, x_{2}, \ldots, x_{n}\right) \in A_{\zeta}\right\}$ (recall that $A_{\zeta}$ lies in $\mathbf{R}^{n}$ ). For the whole length of this proof, vectors of the form $(\alpha, \mathbf{a})$ have $\alpha$ in $\mathbf{R}$ and $\mathbf{a}$ in $\mathbf{R}^{n-1}$.

We use $\Psi$ to show that for $v<x<w, A_{\leq x}$ is nonempty and semi-algebraically connected. Let thus $x$ be fixed in $(v, w)$, and let $(\zeta, \mathbf{z})$ be in $A_{\zeta}$. Remark that $\Psi^{-1}(x, \mathbf{z})$ is in $A_{x}$, proving that $A_{\leq x}$ is nonempty. To prove the connectedness, we use a similar process. Let $\mathbf{y}$ and $\mathbf{y}^{\prime}$ be in $A_{\leq x}$. Since $A$ is semi-algebraically connected, there exists a continuous path $\gamma:[0,1] \rightarrow A$, with $\gamma(t)=(\alpha(t)$, a $(t))$ that connects them. Let us replace $\gamma$ by the path $g$ defined as follows:

- $g(t)=\gamma(t)$ if $\alpha(t) \leq x$;

- $g(t)=\Psi^{-1}(x, \psi(\alpha(t), \mathbf{a}(t)))$ if $a(t) \geq x$.

The path $g(t)$ is well defined, lies in $A_{\leq x}$ by construction, and connects $\mathbf{y}$ to $\mathbf{y}^{\prime}$. This establishes our connectivity claim.

Now, we can deal with the situation above $v$. We cannot directly use the fibration above $v$, since it is not defined above $v$; instead, we will use a limiting process that will rely on semi-algebraicity. To prove that $A_{\leq v}$ is nonempty, we actually prove that $A_{v}$ is. We define the function $\gamma:[0,1) \rightarrow A_{\leq x}$ by $\gamma(t)=\Psi^{-1}(t v+(1-t) \zeta, \mathbf{z})$. This is a semi-algebraic, continuous, bounded function, so it can be extended by continuity at $t=1$ [10, Proposition 3.18]; one checks that $\gamma(1)$ is in $A_{v}$, as requested. 
It remains to prove that $A_{\leq v}$ is semi-algebraically connected. Let thus $\mathbf{y}$ and $\mathbf{y}^{\prime}$ be two points in $A_{\leq v}$. Since $A_{\leq \zeta}$ is semi-algebraically connected (first part of the proof) and semi-algebraic, $\mathbf{y}$ and $\mathbf{y}^{\prime}$ can be connected by a semi-algebraic path $\gamma$ in $A_{\leq \zeta}$ with $\gamma(t)=(\alpha(t), \mathbf{a}(t))$. As we did previously, we replace $\gamma$ by a better path $g$. Let $\varepsilon$ be an infinitesimal, let $A^{\prime}$ be the extension of $A$ over $\mathbf{R}\langle\varepsilon\rangle$, and let $g$ be the path $[0,1] \subset \mathbf{R}\langle\varepsilon\rangle \rightarrow A_{(v, w)}^{\prime}$ defined as follows:

- $g(t)=\gamma(t)$ if $\alpha(t) \leq v+\varepsilon$;

- $g(t)=\Psi^{-1}(v+\varepsilon, \psi(\alpha(t), \mathbf{a}(t)))$ if $\alpha(t) \geq v+\varepsilon$.

Obviously, $g$ is well defined, continuous, bounded over $\mathbf{R}$, and semi-algebraic. Its image $G$ is thus a connected semi-algebraic set, contained in $A_{\leq v+\varepsilon}^{\prime}$. Let $G_{0}=\lim _{\varepsilon} G$. By construction, $\mathbf{y}$ and $\mathbf{y}^{\prime}$ are in $G_{0}, G_{0}$ is contained in $A_{\leq v}$, and by [10, Proposition 12.43], $G_{0}$ is semi-algebraically connected. Our claim follows.

Corollary 13 Let $Z \subset \mathbf{C}^{n}$ be an algebraic set, equidimensional of positive dimension, and such that $Z \cap \mathbf{R}^{n}$ is bounded. Let $v<w$ be in $\mathbf{R}$ such that $Z_{(v, w]} \cap$ $\operatorname{crit}\left(\Pi_{1}, Z\right)=\emptyset$, and let $C$ be a semi-algebraically connected component of $Z_{\leq w}$. Then, $C_{\leq v}$ is a semi-algebraically connected component of $Z_{\leq v}$.

Proof It suffices to prove that $C_{\leq v}$ is nonempty and semi-algebraically connected; then it is easily seen to be a semi-algebraically connected component of $Z_{\leq v}$. If $C_{(v, w]}$ is empty, $C_{\leq v}=C$, so we are done. Hence, we assume that $C_{(v, w]}$ is nonempty.

We verify here that all assumptions of Proposition 12 are satisfied with $A=C_{<w}$. Since $C_{(v, w]}$ is nonempty and $Z_{w} \cap \operatorname{crit}\left(\Pi_{1}, Z\right)$ is empty, $C_{(v, w)}$ is nonempty: either there is a point in $C_{(v, w)}$, or there is a point in $C_{w}$; this point is not in $\operatorname{crit}\left(\Pi_{1}, Z\right)$, so Lemma 7 shows that $C_{(v, w)}$ is not empty in this case as well. Besides, since $Z_{w} \cap$ $\operatorname{crit}\left(\Pi_{1}, Z\right)$ is empty, by Corollary $11, C_{<w}$ is semi-algebraically connected.

Besides, we claim that $\Pi_{1}$ is a submersion on $C_{(v, w)}$. First, remark that for any point $\mathbf{x}$ of $C_{(v, w)}, T_{\mathbf{x}} C_{(v, w)}=T_{\mathbf{x}} Z \cap \mathbf{R}^{n}$. Since $\operatorname{dim}(Z)>0$, and since there is no point of $\operatorname{crit}\left(\Pi_{1}, Z\right)$ on $Z_{(v, w)}$, we know that $\Pi_{1}\left(T_{\mathbf{x}} Z\right)=\mathbf{C}$, which implies that $\Pi_{1}\left(T_{\mathbf{x}} Z \cap\right.$ $\left.\mathbf{R}^{n}\right)=\mathbf{R}$. This establishes that $\Pi_{1}$ is a submersion on $C_{(v, w)}$.

To summarize, $C_{<w}$ is a connected and bounded semi-algebraic set; $C_{(v, w)}$ is a nonempty Nash manifold, closed in $(v, w) \times \mathbf{R}^{n-1}$ (because $C_{(v, w)}=C \cap((v, w) \times$ $\mathbf{R}^{n-1}$ ) and $C$ is closed). We can thus apply Proposition 12, which implies that $C_{\leq v}$ is nonempty and semi-algebraically connected, as requested.

\section{Main Connectivity Result}

\subsection{Initial Form}

In this section, we consider a system $\mathbf{f}=\left(f_{1}, \ldots, f_{p}\right)$ in $\mathbf{R}\left[X_{1}, \ldots, X_{n}\right]$ with $p<n$. We say that the system $\mathbf{f}$ satisfies assumption $\mathbf{H}$ if

(a) the ideal $\left\langle f_{1}, \ldots, f_{p}\right\rangle$ is radical;

(b) $V=V\left(f_{1}, \ldots, f_{p}\right)$ is equidimensional of positive dimension $d=n-p>0$; 
(c) $\operatorname{sing}(V)$ is finite;

(d) $V \cap \mathbf{R}^{n}$ is bounded.

These conditions are independent of the choice of coordinates. Next, assuming that $d \geq 2$, we fix $i$ in $\{2, \ldots, d\}$ and introduce further conditions on $\mathbf{f}$; some are meant to ensure good geometric properties, while some others (e.g., the last one) will help us write our algorithms.

To state these further assumptions, we point out or recall a few facts. First, an equidimensional algebraic set $X$ of dimension $r$ is in Noether position for the projection $\Pi_{r}$ if the extension $\mathbf{C}\left[X_{1}, \ldots, X_{r}\right] \rightarrow \mathbf{C}\left[X_{1}, \ldots, X_{n}\right] / I(X)$ is injective and integral. If this is the case, for any $\mathbf{x}$ in $\mathbf{C}^{r}$, the fiber $X \cap \Pi_{r}^{-1}(\mathbf{x})$ has dimension zero. Next, under $\mathbf{H}$, recall that $\operatorname{crit}\left(\Pi_{i}, V\right)$ is defined by the vanishing of $\mathbf{f}$ and the set $\Delta$ of all $p$-minors of $\operatorname{jac}\left(\mathbf{f},\left[X_{i+1}, \ldots, X_{n}\right]\right)$. Then, we say that $\mathbf{f}$ satisfies condition $\mathbf{H}_{i}^{\prime}$ if the following holds:

(a) $V$ is in Noether position for $\Pi_{d}$;

(b) either $W_{i}$ is empty, or $W_{i}$ is $(i-1)$-equidimensional and in Noether position for $\Pi_{i-1}$

(c) $\operatorname{crit}\left(\Pi_{1}, V\right)$ is finite;

(d) $\operatorname{crit}\left(\Pi_{1}, W_{i}\right)$ is finite;

(e) for $\mathbf{x}$ in $W_{i}-\operatorname{sing}(V), \operatorname{jac}_{\mathbf{x}}\left([\mathbf{f}, \Delta],\left[X_{1}, \ldots, X_{n}\right]\right)$ has rank $n-(i-1)$.

We will see that these new assumptions can be ensured by a generic change of variables for some values of $p$ and $i$ (but not all). Finally, we consider a finite subset of points $\mathcal{P}$ in $V$; with this convention, we define

- $\mathcal{C}=\operatorname{crit}\left(\Pi_{1}, V\right) \cup \operatorname{crit}\left(\Pi_{1}, W_{i}\right) \cup \mathcal{P}$, which is finite under $\mathbf{H}$ and $\mathbf{H}_{i}^{\prime}$;

- $\mathcal{C}^{\prime}=\Pi_{i-1}(\mathcal{C})$;

- $\mathcal{C}^{\prime \prime}=V \cap \Pi_{i-1}^{-1}\left(\mathcal{C}^{\prime}\right)$.

The following theorem is the key to our algorithms. Some properties just repeat the assumptions above; this is in anticipation of an extended version of the theorem (in the next section), where such repetitions will actually be useful.

Theorem 14 Under assumptions $\mathbf{H}$ and $\mathbf{H}_{i}^{\prime}$, the following holds:

1. $W_{i}$ is either empty or $(i-1)$-equidimensional;

2. $\mathcal{C}$ is finite;

3. $\mathcal{C}^{\prime \prime}$ is either empty or $(d-i+1)$-equidimensional;

4. $\mathcal{C}^{\prime \prime} \cup W_{i}$ is a roadmap of $(V, \mathcal{P})$;

5. $\mathcal{C}^{\prime \prime} \cap W_{i}=W_{i} \cap \Pi_{i-1}^{-1}\left(\mathcal{C}^{\prime}\right)$ is finite;

6. for all $\mathbf{x} \in \mathbf{C}^{i-1}$, the system $\left(f_{1}, \ldots, f_{p}, X_{1}-x_{1}, \ldots, X_{i-1}-x_{i-1}\right)$ satisfies assumption $\mathbf{H}$.

This section is devoted to prove this theorem. Once this is done, the idea of our algorithm will roughly be to compute the sets $W_{i}$ and $\mathcal{C}^{\prime \prime}$ and to recursively compute roadmaps of them if their dimension is too high. 


\subsection{First Elements of the Proof}

We start by proving the last two points in the theorem; the other properties will follow easily. The proof uses the following lemma.

Lemma 15 Let $\left(g_{1}, \ldots, g_{p}\right) \subset \mathbf{C}\left[X_{1}, \ldots, X_{n}\right]$, let I be the ideal $\left\langle g_{1}, \ldots, g_{p}\right\rangle \subset$ $\mathbf{C}\left[X_{1}, \ldots, X_{n}\right]$, let $Z$ be its zero-set, and let finally $X$ be the constructible set

$$
X=\left\{\mathbf{x} \in Z \mid \operatorname{rank}\left(\operatorname{jac}_{\mathbf{x}}\left(\mathbf{g},\left[X_{1}, \ldots, X_{n}\right]\right)\right)=p\right\} .
$$

Suppose that $Z$ is not empty and that $X$ is Zariski-dense in $Z$. Then, $I$ is an equidimensional radical ideal of dimension $n-p$.

Proof Since $I$ is generated by $p$ elements of $\mathbf{C}\left[X_{1}, \ldots, X_{n}\right]$, all the primes associated to $I$ have dimension greater than or equal to $n-p$ by Krull's theorem. Since $X$ is dense in $Z, \operatorname{dim}(X)=\operatorname{dim}(Z)$. Moreover, by the implicit function theorem, $\operatorname{dim}(X)=n-p$. Thus, $\operatorname{dim}(I)=n-p$, and $I$ is a complete intersection.

Let $Q_{1} \cap \cdots \cap Q_{s}$ be an irredundant primary decomposition of $I$, so that all associated primes of the $Q_{i}$ are pairwise distinct. Since $\operatorname{dim}(I)=n-p$ and $I$ is generated by $p$ elements, all $Q_{i}$ are isolated by Macaulay's unmixedness theorem [39, Theorem 26, p. 196 (vol. 2)]. Thus, $I$ is unmixed.

We prove below that each $Q_{i}$ is prime, which will imply that $I$ is radical. Since $Q_{i}$ is isolated, its associated algebraic variety is an irreducible component of $Z$ of dimension $n-p$. Besides, $\mathbf{x} \in V\left(Q_{i}\right) \cap V\left(Q_{j}\right) \cap X$ implies that $i=j$.

For $i \leq s$, let $\mathbf{x}$ be in $V\left(Q_{i}\right) \cap X$; such an $\mathbf{x}$ exists since $V\left(Q_{i}\right) \cap X$ is actually dense in all $V\left(Q_{i}\right)$. Let $\mathfrak{m}$ be the maximal ideal at $\mathbf{x}$. Suppose for the moment that $I_{\mathfrak{m}}=Q_{i_{\mathfrak{m}}}$ and $I_{\mathfrak{m}}$ is prime. Then, $Q_{i_{\mathfrak{m}}}$ is obviously prime, which implies that $Q_{i}$ itself is prime by [4, Proposition 3.11(iv)], and we are done.

It remains to prove that $I_{\mathfrak{m}}=Q_{i_{\mathfrak{m}}}$ and $I_{\mathfrak{m}}$ is prime. By [4, Proposition 4.9], $I_{\mathfrak{m}}=$ $Q_{1, \mathfrak{m}} \cap \cdots \cap Q_{s_{\mathfrak{m}}}$. Since we previously proved that $Q_{i}$ is the unique primary ideal of the considered minimal primary decomposition of $I$ such that $\mathbf{x} \in V\left(Q_{i}\right), Q_{i}$ is the unique ideal of that decomposition which is contained in $\mathfrak{m}$. Thus, $I_{\mathfrak{m}}=Q_{i_{\mathfrak{m}}}$.

Part $b$ of [19, Theorem 16.19] shows that the local ring $\mathbf{C}\left[X_{1}, \ldots, X_{n}\right]_{\mathfrak{m}} / I_{\mathfrak{m}}$ is regular and hence an integral ring, so that $I_{\mathfrak{m}}$ is prime.

Lemma 16 Under assumptions $\mathbf{H}$ and $\mathbf{H}_{i}^{\prime}, \mathcal{C}^{\prime \prime} \cap W_{i}$ is finite, and for all $\mathbf{x} \in \mathbf{C}^{i-1}$, the system $\left(f_{1}, \ldots, f_{p}, X_{1}-x_{1}, \ldots, X_{i-1}-x_{i-1}\right)$ satisfies assumption $\mathbf{H}$.

Proof We start with the second point. Consider $\mathbf{x}=\left(x_{1}, \ldots, x_{i-1}\right)$ in $\mathbf{C}^{i-1}$ and let $V_{\mathbf{x}}$ be the algebraic set defined by $\left(f_{1}, \ldots, f_{p}, X_{1}-x_{1}, \ldots, X_{i-1}-x_{i-1}\right)$. Let us show that it is not empty: by $\mathbf{H}_{i}^{\prime}(a), V$ is in Noether position for $\Pi_{d}$, so for any $\mathbf{x}^{\prime}=\left(x_{1}, \ldots, x_{d}\right), V \cap \Pi_{d}^{-1}\left(\mathbf{x}^{\prime}\right)$ is not empty; a fortiori, $V_{\mathbf{x}}=V \cap \Pi_{i-1}^{-1}(\mathbf{x})$ is not empty. By Krull's theorem, we deduce that all irreducible components of $V_{\mathbf{x}}$ have dimension at least $d-(i-1)$.

Let $\mathbf{y}$ be in $V_{\mathbf{x}}$. By construction, if the Jacobian of $\left(f_{1}, \ldots, f_{p}, X_{1}-x_{1}, \ldots\right.$, $\left.X_{i-1}-x_{i-1}\right)$ has not full rank, then $\mathbf{y}$ is in $\operatorname{crit}\left(\Pi_{i}, V\right) \cap \Pi_{i-1}^{-1}(\mathbf{x})$. Recall now that 
$\operatorname{crit}\left(\Pi_{i}, V\right)=W_{i} \cup \operatorname{sing}(V)$. Since by $\mathbf{H}_{i}^{\prime}(b), W_{i}$ is either empty or in Noether position for $\Pi_{i-1}$, we deduce that $W_{i} \cap \Pi_{i-1}^{-1}(\mathbf{x})$ is finite. Since $\operatorname{sing}(V)$ is also finite, $\operatorname{crit}\left(\Pi_{i}, V\right) \cap \Pi_{i-1}^{-1}(\mathbf{x})$ is finite.

Thus, since $d-(i-1) \geq 1$, each irreducible component of $V_{\mathbf{x}}$ contains a point $\mathbf{y}$ where the former Jacobian matrix has full rank. Consequently, we deduce by Lemma 15 that the system $\left(f_{1}, \ldots, f_{p}, X_{1}-x_{1}, \ldots, X_{i-1}-x_{i-1}\right)$ is radical and $(d-i+1)$-equidimensional. We have thus established $\mathbf{H}(a)$ and $\mathbf{H}(b)$ for that system (for $\mathbf{H}(b)$, remark that $d-i+1$ is positive). The singular points of $V_{\mathbf{x}}$ are the points where the rank of the former Jacobian drops; as we have seen, they are in finite number. This gives $\mathbf{H}(c)$. Point $\mathbf{H}(d)$ is obvious, since $V_{\mathbf{x}} \cap \mathbf{R}^{n} \subset V \cap \mathbf{R}^{n}$, and the latter is bounded.

The other assertion follows from the fact that $\mathcal{C}^{\prime \prime} \cap W_{i}$ is the union of the sets $W_{i} \cap \Pi_{i-1}^{-1}(\mathbf{x})$ for $\mathbf{x}$ in $\mathcal{C}^{\prime}=\Pi_{i-1}(\mathcal{C})$. Since these sets are all finite, and since $\mathcal{C}$ and thus $\mathcal{C}^{\prime}$ are finite as well (by $\mathbf{H}_{i}^{\prime}(c)$ and $\left.\mathbf{H}_{i}^{\prime}(d)\right)$, we are done.

To prove Theorem 14, we note that the first two points are either part of $\mathbf{H}_{i}^{\prime}$ or direct consequence thereof. We have seen in the previous lemma that all fibers $\Pi_{i-1}^{-1}(\mathbf{x}) \cap V$ are $(d-i+1)$-equidimensional, for $\mathbf{x} \in \mathbf{C}^{i-1}$. Since $\mathcal{C}^{\prime \prime}$ is the union of such fibers for $\mathbf{x}$ in $\mathcal{C}^{\prime}=\Pi_{i-1}(\mathcal{C})$, then it is either $(d-i+1)$-equidimensional, or empty if $\mathcal{C}$ is empty. This gives the third point. The last two points are in the previous lemma.

All that is missing is thus point 4 . The connectivity property $\mathrm{RM}_{1}^{\prime}$ is established in Sect. 4.3. Property $\mathrm{RM}_{2}^{\prime}$ is clear from the construction; also, $\mathcal{P}$ is contained in $\mathcal{C}$ and thus in $\Pi_{i-1}^{-1}\left(\mathcal{C}^{\prime}\right)$, so we obtain $\mathrm{RM}_{4}^{\prime}$.

\subsection{Proof of Property $\mathrm{RM}_{1}^{\prime}$}

We reuse here the notation of Theorem 14, and we let $\mathcal{R}=\mathcal{C}^{\prime \prime} \cup W_{i}$. For $x$ in $\mathbf{R}$, we say that property $\mathbf{P}(x)$ holds if:

- for any semi-algebraically connected component $C$ of $V_{\leq x}, C \cap \mathcal{R}$ is nonempty and semi-algebraically connected.

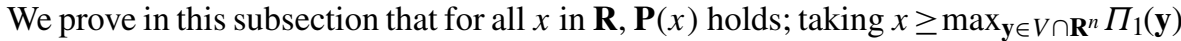
proves property $\mathrm{RM}_{1}^{\prime}$ of Theorem 14 .

Let $v_{1}<\cdots<v_{\ell}$ be the points in $\Pi_{1}(\mathcal{C}) \cap \mathbf{R}$ (recall that $\mathcal{C}$ is finite). The proof uses two intermediate results:

- Step 1: if $\mathbf{P}\left(v_{j}\right)$ holds, then for $x$ in $\left(v_{j}, v_{j+1}\right), \mathbf{P}(x)$ holds;

- Step 2: for $x$ in $\mathbf{R}$, if $\mathbf{P}\left(x^{\prime}\right)$ holds for all $x^{\prime}<x$, then $\mathbf{P}(x)$ holds.

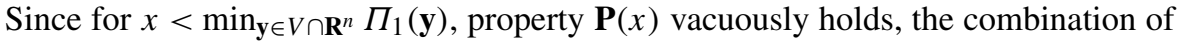
these two results gives the claim above by an immediate induction.

Proposition 17 (Step 1) Let $j$ be in $\{1, \ldots, \ell-1\}$. If $\mathbf{P}\left(v_{j}\right)$ holds, then for $x$ in $\left(v_{j}, v_{j+1}\right), \mathbf{P}(x)$ holds.

Proof Let $x$ be in $\left(v_{j}, v_{j+1}\right)$, and let $C$ be a semi-algebraically connected component of $V_{\leq x}$. We have to prove that $C \cap \mathcal{R}$ is nonempty and semi-algebraically connected. 
We first establish that $C_{\leq v_{j}} \cap \mathcal{R}$ is nonempty and semi-algebraically connected. Because there is no point of $\operatorname{crit}\left(\Pi_{1}, V\right)$ in $V_{\left(v_{j}, x\right]}$, applying Corollary 13 to $V$ above the interval $\left(v_{j}, x\right]$ shows that $C_{\leq v_{j}}$ is a semi-algebraically connected component of $V_{\leq v_{j}}$. So, using property $\mathbf{P}\left(v_{j}\right)$, we see that $C_{\leq v_{j}} \cap \mathcal{R}$ is nonempty and semialgebraically connected, as needed.

Next, we prove that, assuming that $C \cap W_{i}$ is not empty, for any semi-algebraically connected component $D$ of $C \cap W_{i}, D_{\leq v_{j}}$ is nonempty. Clearly, $D$ is a semialgebraically connected component of $W_{i \leq x}$. By assumption $\mathbf{H}_{i}^{\prime}, W_{i}$ is an algebraic set, equidimensional of positive dimension $i-1$, with $W_{i} \cap \mathbf{R}^{n}$ bounded; besides, $\operatorname{crit}\left(\Pi_{1}, W_{i}\right)$ is empty above $\left(v_{j}, x\right]$. Applying Corollary 13 to $W_{i}$, we see that $D_{\leq v_{j}}$ is nonempty (and semi-algebraically connected).

To prove that $C \cap \mathcal{R}$ is semi-algebraically connected, we prove that any $\mathbf{y}$ in $C \cap \mathcal{R}$ can be semi-algebraically connected to a point in $C_{\leq v_{j}} \cap \mathcal{R}$ by a path in $C \cap \mathcal{R}$. This is sufficient to conclude, since we have seen that $C_{\leq v_{j}} \cap \mathcal{R}$ is semi-algebraically connected. Let thus $\mathbf{y}$ be in $C \cap \mathcal{R}$. If $\mathbf{y}$ is in $C_{\leq v_{j}} \cap \mathcal{R}$, we are done. If $\mathbf{y}$ is in $C_{\left(v_{j}, x\right]} \cap \mathcal{R}$, we claim that it is actually in $C_{\left(v_{j}, x\right]} \cap W_{i}$. Indeed, $\mathcal{R}$ and $W_{i}$ coincide above $\left(v_{j}, x\right]$ : for any point $\mathbf{z}$ in $\mathcal{C}^{\prime \prime} \cap \mathbf{R}^{n}, \Pi_{1}(\mathbf{z})$ is in $\Pi_{1}(\mathcal{C}) \cap \mathbf{R}$, so it is one of $v_{1}, \ldots, v_{\ell}$.

Let thus $D$ be the semi-algebraically connected component of $C \cap W_{i}$ containing $\mathbf{y}$. By the result of the previous paragraph, there exists a semi-algebraic continuous path connecting $\mathbf{y}$ to a point $\mathbf{y}^{\prime}$ in $D_{\leq v_{j}}$ by a path in $D$. Since $D$ is in $C \cap \mathcal{R}$, we are done.

Proposition 18 (Step 2) Let $x$ be in $\mathbf{R}$ such that for all $x^{\prime}<x, \mathbf{P}\left(x^{\prime}\right)$ holds. Then $\mathbf{P}(x)$ holds.

Proof Let $C$ be a semi-algebraically connected component of $V_{\leq x}$; we have to prove that $C \cap \mathcal{R}$ is semi-algebraically connected. If $C$ is finite, we are done, since $C$ is a point and $C \cap \mathcal{R}$ is semi-algebraically connected as it is nonempty (one checks that in this case, $C$ is in $\left.\operatorname{crit}\left(\Pi_{1}, V\right)\right)$.

Hence, we assume that $C$ is infinite; from this one deduces that $C_{<x}$ is not empty: since $\operatorname{crit}\left(\Pi_{1}, V\right)$ is finite by $\mathbf{H}_{i}^{\prime}(c)$, there is a point in $C$ not in $\operatorname{crit}\left(\Pi_{1}, V\right)$, and applying Lemma 7 proves our claim. Let then $B_{1}, \ldots, B_{r}$ be the semi-algebraically connected components of $C_{<x}$; we will prove in the next subsection that for $i \leq r$, $\overline{B_{i}} \cap \mathcal{R}$ is nonempty and semi-algebraically connected.

Fix $i \leq r$. Since $\overline{B_{i}} \cap \mathcal{R}$ is nonempty and contained in $C \cap \mathcal{R}$, the latter is nonempty. Let thus $\mathbf{y}$ and $\mathbf{y}^{\prime}$ be in $C \cap \mathcal{R}$; we need to connect them by a path in $C \cap \mathcal{R}$. Let $\gamma:[0,1] \rightarrow C$ be a continuous semi-algebraic path that connects $\mathbf{y}$ to $\mathbf{y}^{\prime}$, and let $G=\gamma^{-1}\left(C_{x} \cap \operatorname{crit}\left(\Pi_{1}, V\right)\right)$ and $H=[0,1]-G$. The semi-algebraically connected components $g_{1}, \ldots, g_{N}$ of $G$ are closed intervals in $[0,1]$ (which may be reduced to single points); the semi-algebraically connected components $h_{1}, \ldots, h_{M}$ of $H$ are intervals that are open in $[0,1]$. For $1 \leq i \leq M$, we write $\ell_{i}=\inf \left(h_{i}\right)$ and $r_{i}=\sup \left(h_{i}\right)$; we also introduce $r_{0}=0$ and $\ell_{M+1}=1$. To conclude the proof, it will be enough to establish that:

1. for $1 \leq i \leq M, \gamma\left(\ell_{i}\right)$ and $\gamma\left(r_{i}\right)$ can be connected by a semi-algebraic path in $C \cap \mathcal{R}$; 
2. for $0 \leq i \leq M, \gamma\left(r_{i}\right)=\gamma\left(\ell_{i+1}\right)$.

We prove the first point. For $1 \leq i \leq M$, we first claim that there exists $j \leq r$ such that $\gamma\left(h_{i}\right)$ is in $\overline{B_{j}}$. Indeed, remark that since $\gamma\left(h_{i}\right)$ avoids $C_{x} \cap \operatorname{crit}\left(\Pi_{1}, V\right)$, it actually avoids the whole $V_{x} \cap \operatorname{crit}\left(\Pi_{1}, V\right)$ (because $\gamma\left(h_{i}\right)$ is contained in $C$ ). It follows from Proposition 5 that there exists a semi-algebraically connected component $B$ of $V_{<x}$ such that $\gamma\left(h_{i}\right) \subset \bar{B}$. One checks that $B$ is actually a semi-algebraically connected component of $C_{<x}$ (one first deduces that $C \cap \bar{B}$ is not empty, so that $C \cap B$ is not empty either, and the conclusion follows). Thus, we will rewrite $B=B_{j}$ for some $j \leq r$.

Since $\gamma$ is continuous, both $\gamma\left(\ell_{i}\right)$ and $\gamma\left(r_{i}\right)$ are in $\overline{B_{j}}$. On the other hand, both $\gamma\left(\ell_{i}\right)$ and $\gamma\left(r_{i}\right)$ are in $\mathcal{R}$. We justify it for $\ell_{i}$ : either $\ell_{i}=0$, and we are done (because $\gamma(0)=\mathbf{y}$ is in $\mathcal{R}$ ), or $\ell_{i}>0$, so that $\ell_{i}$ is in some interval $g_{\ell}$ (since then it does not belong to $\left.h_{i}\right)$, and thus $\gamma\left(\ell_{i}\right)$ is in $\operatorname{crit}\left(\Pi_{1}, V\right) \subset \mathcal{R}$. Because $\overline{B_{j}} \cap \mathcal{R}$ is semialgebraically connected, $\gamma\left(\ell_{i}\right)$ and $\gamma\left(r_{i}\right)$ can be connected by a semi-algebraic path in $\overline{B_{j}} \cap \mathcal{R}$, which is contained in $C \cap \mathcal{R}$.

The second point is easier to deal with. If $r_{i}=\ell_{i+1}$ (which can happen at $r_{0}=0$ or $\ell_{M+1}=1$ ), the conclusion holds trivially. Else, we have $r_{i}<\ell_{i+1}$; then, both are in the same interval $g_{\ell}$ for some $\ell \leq N$. Since $\operatorname{crit}\left(\Pi_{1}, V\right)$ is finite, $\gamma\left(g_{\ell}\right)$ is a single point (since it is semi-algebraically connected), so $\gamma\left(r_{i}\right)=\gamma\left(\ell_{i+1}\right)$.

\subsection{Conclusion}

We deal here with the following statement from the previous subsection: as above, let $C$ be a semi-algebraically connected component of $V \cap \mathbf{R}^{n}$. Let $B$ be one of the semi-algebraically connected components of $C_{<x}$. We have to prove that $\bar{B} \cap \mathcal{R}$ is nonempty and semi-algebraically connected.

Since $B$ is actually a semi-algebraically connected component of $V_{<x}$ and $V \cap \mathbf{R}^{n}$ is bounded, $B$ contains a point of $\operatorname{crit}\left(\Pi_{1}, V\right)$ (the point at which $\Pi_{1}$ reaches its minimum on $B$ ). Hence, $B \cap \mathcal{R}$, and thus $\bar{B} \cap \mathcal{R}$, are not empty. Next, we prove that any point $\mathbf{y}$ in $\bar{B} \cap \mathcal{R}$ can be connected to a point $\mathbf{z}$ in $B \cap \mathcal{R}$ by a semi-algebraic path in $\bar{B} \cap \mathcal{R}$. Assuming that this is the case, let us first justify that this is sufficient to establish the lemma.

Consider two points $\mathbf{y}, \mathbf{y}^{\prime}$ in $\bar{B} \cap \mathcal{R}$ and suppose that they can be connected to some points $\mathbf{z}, \mathbf{z}^{\prime}$ in $B \cap \mathcal{R}$ by semi-algebraic paths in $\bar{B} \cap \mathcal{R}$. Since $\mathbf{z}$ and $\mathbf{z}^{\prime}$ are in $B$, they can be connected by a semi-algebraic path $\gamma:[0,1] \rightarrow B$. Let $x^{\prime}=\max \left(\Pi_{1}(\gamma(t))\right)$ for $t$ in $[0,1] ; x^{\prime}$ is well defined by the continuity of $\gamma$ and satisfies $x^{\prime}<x$. Then, both $\mathbf{z}$ and $\mathbf{z}^{\prime}$ are in $B_{\leq x^{\prime}}$, and they can be connected by a semi-algebraic path in $B_{\leq x^{\prime}}$; hence, they are in the same semi-algebraically connected component $B^{\prime}$ of $B_{\leq x^{\prime}}$. Now, $B^{\prime}$ is a semi-algebraically connected component of $V_{\leq x^{\prime}}$, which implies by property $\mathbf{P}\left(x^{\prime}\right)$ that $B^{\prime} \cap \mathcal{R}$ is semi-algebraically connected. Hence, $\mathbf{z}$ and $\mathbf{z}^{\prime}$, which are in $B^{\prime} \cap \mathcal{R}$, can be connected by a semi-algebraic path in $B^{\prime} \cap \mathcal{R}$ and thus within $B \cap \mathcal{R}$. Summarizing, this proves that $\mathbf{y}$ and $\mathbf{y}^{\prime}$ can be connected by a semi-algebraic path in $\bar{B} \cap \mathcal{R}$, as requested.

We are thus left to prove the claim made in the first paragraph. Recall that $\mathcal{R}$ is the union of $W_{i}$ and of $\mathcal{C}^{\prime \prime}=V \cap \Pi_{i-1}^{-1}\left(\Pi_{i-1}(\mathcal{C})\right)$, where $\mathcal{C}=\operatorname{crit}\left(\Pi_{1}, V\right) \cup$ 
$\operatorname{crit}\left(\Pi_{1}, W_{i}\right) \cup \mathcal{P}$. We first deal with points $\mathbf{y}$ in $\bar{B} \cap \mathcal{C}^{\prime \prime}$ and then, secondly, with points $\mathbf{y}$ in $\bar{B} \cap\left(W_{i}-\mathcal{C}^{\prime \prime}\right)$.

Case 1 . Let $\mathbf{y}$ be in $\bar{B} \cap \mathcal{C}^{\prime \prime}$. We can assume that $\mathbf{y}$ is not in $B$, since for $\mathbf{y}$ in $B$, we can take $\mathbf{z}=\mathbf{y}$; since $\mathbf{y}$ is not in $B, \Pi_{1}(\mathbf{y})=x$.

Since $B$ is semi-algebraic, by the curve selection lemma, there exists a continuous semi-algebraic map $f:[0,1] \rightarrow \mathbf{R}^{n}$ with $f(0)=\mathbf{y}$ and $f(t) \in B$ for $t$ in $(0,1]$. Let $\varepsilon$ be a new infinitesimal, and let $\mathbf{R}^{\prime}=\mathbf{R}\langle\varepsilon\rangle$; we let $\varphi=\left(\varphi_{1}, \ldots, \varphi_{n}\right) \in \mathbf{R}^{\prime \prime}$ be the semi-algebraic germ of $f$ at 0 , so that $\lim _{\varepsilon} \varphi=\mathbf{y}$. We consider the semi-algebraic set $H \subset \mathbf{R}^{\prime n}$ defined by

$$
H=\left\{\mathbf{x} \in \mathbf{R}^{\prime n} \mid \mathbf{x} \in \operatorname{ext}\left(B, \mathbf{R}^{\prime}\right) \text { and }\left(x_{1}, \ldots, x_{i-1}\right)=\left(\varphi_{1}, \ldots, \varphi_{i-1}\right)\right\},
$$

where ext denotes the extension to $\mathbf{R}^{\prime}$. Since for all $t$ in $(0,1], f(t)$ is in $B, \varphi$ is in $\operatorname{ext}\left(B, \mathbf{R}^{\prime}\right)$ by [10, Proposition 3.16], so that $\varphi$ is in $H$; in particular, this proves that $\mathbf{y}$ is in $\lim _{\varepsilon} H$. Remark also that $H$ is bounded by an element of $\mathbf{R}$ and that any point in $\lim _{\varepsilon} H$ is in $\bar{B} \cap \Pi_{i-1}^{-1}\left(\Pi_{i-1}(\mathbf{y})\right)$, which is contained in $\bar{B} \cap \mathcal{R}$ by assumption on $\mathbf{y}$.

Let $H_{1}, \ldots, H_{s} \subset \mathbf{R}^{\prime n}$ be the semi-algebraically connected components of $H$ (which are well defined because $H$ is not empty); hence, the $H_{i}$ are semi-algebraic sets. Because $\mathbf{y}$ is in $\lim _{\varepsilon}(H)$, we can assume that it is in $\lim _{\varepsilon} H_{1}$. Next, since $B$ is a semi-algebraically connected component of $V_{<x}$, by [10, Proposition 5.24], $\operatorname{ext}\left(B, \mathbf{R}^{\prime}\right)$ is a semi-algebraically connected component of $\operatorname{ext}\left(V, \mathbf{R}^{\prime}\right)_{<x}$, which implies that $H_{1}$ is a semi-algebraically connected component of $\operatorname{ext}\left(V, \mathbf{R}^{\prime}\right) \cap$ $\Pi_{i-1}^{-1}\left(\varphi_{1}, \ldots, \varphi_{i-1}\right)$.

Since $H_{1}$ is bounded, by the semi-algebraic implicit function theorem [10, Theorem 3.25], this implies that there exists a point $\psi$ in $H_{1} \cap \operatorname{crit}\left(\Pi_{i}, \operatorname{ext}\left(V, \mathbf{R}^{\prime}\right)\right)$. Since polar varieties are defined by Jacobian minors with coefficients in $\mathbf{R}$, this means that $\psi$ is in $H_{1} \cap \operatorname{ext}\left(\operatorname{crit}\left(\Pi_{i}, V\right), \mathbf{R}^{\prime}\right)$. Because $\psi$ is in $H_{1}$, it is in $\operatorname{ext}\left(B, \mathbf{R}^{\prime}\right)$ and thus in $\operatorname{ext}\left(B \cap \operatorname{crit}\left(\Pi_{i}, V\right), \mathbf{R}^{\prime}\right)$.

Let $\mathbf{w}=\lim _{\varepsilon} \psi$, and let $g$ be a representative of $\psi$, so that $g(0)=\mathbf{w}$. By $[10$, Proposition 3.16], there exists $t_{0}>0$ such that for all $t$ in $\left(0, t_{0}\right), g(t)$ is in $B \cap$ $\operatorname{crit}\left(\Pi_{i}, V\right)$. Remark next that $\operatorname{crit}\left(\Pi_{i}, V\right)$ is contained in $\mathcal{R}$ : any point in $\operatorname{crit}\left(\Pi_{i}, V\right)$ is either in $\operatorname{sing}(V)$ (in which case it is in $\left.\operatorname{crit}\left(\Pi_{1}, V\right) \subset \mathcal{R}\right)$ or in $W_{i} \subset \mathcal{R}$. Thus, for all $t$ in $\left[0, t_{0}\right], g(t)$ is in $\bar{B} \cap \mathcal{R}$. Defining $\mathbf{z}=g\left(t_{0} / 2\right)$, we see that $\mathbf{z}$ and $\mathbf{w}$ are connected by a semi-algebraic path in $\bar{B} \cap \mathcal{R}$.

Let $B_{1}=\lim _{\varepsilon} H_{1}$. Because $H_{1}$ is semi-algebraic, bounded over $\mathbf{R}$, and semialgebraically connected, $B_{1}$ is closed, semi-algebraic, and semi-algebraically connected [10, Proposition 12.43]. Besides, we have seen above that it is contained in $\bar{B} \cap \mathcal{R}$. Finally, it contains both $\mathbf{y}$ and $\mathbf{w}$. Connecting $\mathbf{y}$ to $\mathbf{w}$ and $\mathbf{w}$ to $\mathbf{z}$ (previous paragraph), we conclude the proof of our claim.

Case 2. Let now $\mathbf{y}$ be in $\bar{B} \cap\left(W_{i}-\mathcal{C}^{\prime \prime}\right)$; as in case 1 , we assume that $\mathbf{y}$ is not in $B$, so that $\Pi_{1}(\mathbf{y})=x$. Since $\mathbf{y}$ is not in $\mathcal{C}^{\prime \prime}, \mathbf{y}$ is not in $\mathcal{C}$ and so not in $\operatorname{crit}\left(\Pi_{1}, W_{i}\right)$. Applying Lemma 7 to the algebraic set $W_{i}$, we see that $\mathbf{y}$ is in $\overline{W_{i<x}}$. By the curve selection lemma, this means that there exists a semi-algebraic path $\gamma:[0,1] \rightarrow W_{i}$ connecting a point $\mathbf{z}$ in $W_{i<x}$ to $\mathbf{y}$ with $\gamma(0)=\mathbf{z}, \gamma(1)=\mathbf{y}$, and $\gamma(t) \in W_{i<x}$ for $t<1$.

The image of $\gamma$ is in $\mathcal{R}$, so to conclude, it suffices to prove that $\gamma(t)$ is in $\bar{B}$ for all $t$. To do so, we will prove that $\gamma(t)$ is in $B$ for all $t<1$. We know that the image 
$\{\gamma(t) \mid t \in[0,1)\}$ is semi-algebraically connected and contained in $V_{<x}$; hence, it is contained in a semi-algebraically connected component $B^{\prime}$ of $V_{<x}$. We have to prove that $B^{\prime}=B$. Because $\gamma(1)=\mathbf{y}$, we deduce that $\mathbf{y}$ is in $\overline{B^{\prime}}$; on the other hand, we know that $\mathbf{y}$ is in $\bar{B}$. Since $\mathbf{y}$ is not in $\mathcal{C}$, it is not in $\operatorname{crit}\left(\Pi_{1}, V\right)$; as a consequence, we can apply Lemma 8 , which shows that $B=B^{\prime}$, as requested.

\section{Algorithms}

\subsection{Overview}

Consider a polynomial system $\mathbf{f}=\left(f_{1}, \ldots, f_{p}\right) \subset \mathbf{R}\left[X_{1}, \ldots, X_{n}\right]$ defining an algebraic set $V$ of dimension $d=n-p>0$ and satisfying $\mathbf{H}$, and a finite set of control points $\mathcal{P}$ in $\mathbf{C}^{n}$. We will see hereafter that if $d \geq 2$, then for some values of $p$ and $2 \leq i \leq d$ (but not all), $\mathbf{H}_{i}^{\prime}$ can be ensured by a generic linear change of variables.

Supposing that $\mathbf{H}_{i}^{\prime}$ holds, one can apply Theorem 14 to obtain a roadmap of $(V, \mathcal{P})$ of dimension at most $\max (i-1, d-i+1)$. Note that this roadmap is given as the union of two algebraic sets $\mathcal{R}_{1}$ and $\mathcal{R}_{2}$ :

- if it is not empty, $\mathcal{R}_{1}$ is the algebraic set $W_{i}$, which is $(i-1)$-equidimensional;

- if it is not empty, $\mathcal{R}_{2}$ is defined by a pair [f, $\left.Q\right]$, where $Q=Q\left(X_{1}, \ldots, X_{i-1}\right)$ is a zero-dimensional parameterization: $\mathcal{R}_{2}=V \cap \Pi_{i-1}^{-1}(Z(Q))$ is the union of the fibers $V \cap \Pi_{i-1}^{-1}(\mathbf{x})$ for $\mathbf{x} \in Z(Q)$; it is $(d-i+1)$-equidimensional.

For the sake of discussion, let us assume that neither $\mathcal{R}_{1}$ nor $\mathcal{R}_{2}$ is empty. Using Proposition 2 , it is natural to compute a 1-roadmap $\mathcal{R}_{1}^{\prime}$ of $\left(\mathcal{R}_{1},\left(\mathcal{R}_{1} \cap \mathcal{R}_{2}\right) \cup \mathcal{P}\right)$ and a 1-roadmap $\mathcal{R}_{2}^{\prime}$ of $\left(\mathcal{R}_{2},\left(\mathcal{R}_{1} \cap \mathcal{R}_{2}\right) \cup \mathcal{P}\right)$ in order to construct a 1-roadmap of $(V, \mathcal{P})$. Suppose that one can construct systems defining $\mathcal{R}_{1}$ and $\mathcal{R}_{2}$ satisfying $\mathbf{H}$. Once again, we are led to use Theorem 14 to compute them; this is possible only if $\mathbf{H}_{i}^{\prime}$ can be ensured for some $i^{\prime}$ in respectively $\left\{2, \ldots, \operatorname{dim}\left(\mathcal{R}_{1}\right)\right\}$ and $\left\{2, \ldots, \operatorname{dim}\left(\mathcal{R}_{2}\right)\right\}$.

Thus, algorithms based on Theorem 14 are naturally recursive. Due to this recursive nature, we will have to handle pairs [f, $Q]$, where $Q=Q\left(X_{1}, \ldots, X_{e}\right)$ is a zero-dimensional parameterization. We will be interested in the algebraic set $V([\mathbf{f}, Q])=V(\mathbf{f}) \cap \Pi_{e}^{-1}(Z(Q))$ : this means that we will restrict $X_{1}, \ldots, X_{e}$ to a finite number of possible values that are described by $Q$. In order to apply Theorem 14 , we need an extended form of this latter result, by defining analogues of assumptions $\mathbf{H}$ and $\mathbf{H}_{i}^{\prime}$ in this context. This is done in Sect. 5.2.

We will see that the degrees of the output roadmaps and the running time necessary to compute them depend on the depth of the recursion. Thus, we are led to reduce as much as possible the depth of the recursion: the best we could hope for is $\simeq \log (n)$. However, one has to ensure $\mathbf{H}_{i}^{\prime}$ each time we apply Theorem 14 in its extended form; this constraints our possible choices. More precisely, we will prove in Sect. 6 that

(a) for a system of equations $\mathbf{f}=\left(f_{1}, \ldots, f_{p}\right)$, if [f $\left.\mathbf{f}, Q\right]$ satisfies $\mathbf{H}$, then $\mathbf{H}_{2}^{\prime}$ can be ensured by a generic linear change of variables leaving $X_{1}, \ldots, X_{e}$ fixed;

(b) for a single equation $f$, if $[f, Q]$ satisfies $\mathbf{H}$, then, for any $2 \leq i \leq n-e-1, \mathbf{H}_{i}^{\prime}$ can ensured by a generic linear change of variables leaving $X_{1}, \ldots, X_{e}$ fixed. 
The algorithmic by-products of (a) and (b) are twofold:

- A subroutine CannyRoadmap-described in Sect. 5.4 and which is close to Canny's algorithm - taking as input [f, $Q$ ] (which satisfies $\mathbf{H}$ ) as above and a finite set of control points $\mathcal{P}$; it applies recursively Theorem 14 in its extended form with $i=2$; this routine performs baby steps by constructing roadmaps whose dimensions decrease one by one.

- A subroutine MainRoadmap—described in Sect. 5.5-which takes as input $[f, Q]$ (which satisfies $\mathbf{H}$ ) as above and a finite set of control points $\mathcal{P}$; it applies recursively Theorem 14 in its extended form with $i \simeq \sqrt{n}$; this routine performs giant steps by producing two algebraic sets $\mathcal{R}_{1}$ and $\mathcal{R}_{2}$ of respective dimensions $\simeq \sqrt{n}-e$ and $\simeq n-\sqrt{n}-e$; then CannyRoadmap is called recursively on $\mathcal{R}_{1}$ while MainRoadmap is called recursively on $\mathcal{R}_{2}$.

These subroutines use procedures performing basic algebraic elimination operations for solving polynomial systems, or manipulating zero- or one-dimensional algebraic sets (to compute unions, projections, ....). These procedures are described in Sect. 5.3, but the proofs are postponed to the end of the section, in Sect. 5.6.

\subsection{Connectivity result: extended form}

As explained above, due to the recursive nature of the algorithm, we handle pairs $[\mathbf{f}, Q]$ with $\mathbf{f}=\left(f_{1}, \ldots, f_{p}\right)$ and a zero-dimensional parameterization $Q\left(X_{1}, \ldots, X_{e}\right)$. This subsection is devoted to obtain an extension of Theorem 14 to such inputs. Recall that we write $V=V(\mathbf{f})$; we will write $V([\mathbf{f}, Q])$ to mean $V \cap \Pi_{e}^{-1}(Z(Q))$. As before, we are also given a set of control points $\mathcal{P}$.

In this new context, we define analogues of $\mathbf{H}$ and $\mathbf{H}_{i}^{\prime}$. For $\mathbf{x}=\left(x_{1}, \ldots, x_{e}\right)$ in $\mathbf{C}^{e}$ and $1 \leq j \leq p$, we let $f_{j, \mathbf{x}}=f_{j}\left(x_{1}, \ldots, x_{e}, X_{e+1}, \ldots, X_{n}\right)$, and we let $\mathbf{f}_{\mathbf{x}}$ be the system $\mathbf{f}_{\mathbf{x}}=\left(f_{1, \mathbf{x}}, \ldots, f_{p, \mathbf{x}}\right)$. Then, we say that $[\mathbf{f}, Q]$ satisfies $\mathbf{H}$ if for all $\mathbf{x}$ in $Z(Q)$, the system $\mathbf{f}_{\mathbf{x}}$ satisfies $\mathbf{H}$ in $\mathbf{C}\left[X_{e+1}, \ldots, X_{n}\right]$; in particular, $V([\mathbf{f}, Q])$ is equidimensional of dimension $d=n-e-p>0$. Assume further that $d \geq 2$ and fix an integer $i$ in $\{2, \ldots, d\}$. Then, we say that $[\mathbf{f}, Q]$ satisfies $\mathbf{H}_{i}^{\prime}$ if for all $\mathbf{x}$ in $Z(Q)$, the system $\mathbf{f}_{\mathbf{x}}$ satisfies $\mathbf{H}_{i}^{\prime}$ in $\mathbf{C}\left[X_{e+1}, \ldots, X_{n}\right]$.

These assumptions describe geometric conditions in $\mathbf{C}^{n-e}$. In $\mathbf{C}^{n}$, since we restrict the first $e$ coordinates to a finite set, it is now natural to define the projection

$$
\begin{aligned}
\Pi_{e, i}: & \mathbf{C}^{n} \rightarrow \mathbf{C}^{i}, \\
\quad \mathbf{x} & =\left(x_{1}, \ldots, x_{n}\right) \mapsto\left(x_{e+1}, \ldots, x_{e+i}\right),
\end{aligned}
$$

so that $\Pi_{i}=\Pi_{0, i}$. Extending the previous notation, we define $w_{i, Q} \subset \mathbf{C}^{n}$ as the set of all critical points of $\Pi_{e, i}$ on $\operatorname{reg}(V([\mathbf{f}, Q]))$, and let $W_{i, Q}$ be its Zariski-closure in $\mathbf{C}^{n}$.

We will verify later on that, under $\mathbf{H}$ and $\mathbf{H}_{i}^{\prime}$, the set $W_{i, Q}$ is either empty or $(i-1)$-equidimensional, so that $\operatorname{crit}\left(\Pi_{e, 1}, W_{i, Q}\right)$ makes sense. Then, we define

- $\mathcal{P}_{Q}=\mathcal{P} \cap \Pi_{e}^{-1}(Z(Q))$;

- $\mathcal{C}_{Q}=\operatorname{crit}\left(\Pi_{e, 1}, V([\mathbf{f}, Q])\right) \cup \operatorname{crit}\left(\Pi_{e, 1}, W_{i, Q}\right) \cup \mathcal{P}_{Q}$;

- $\mathcal{C}_{Q}^{\prime}=\Pi_{e+i-1}\left(\mathcal{C}_{Q}\right)$;

- $\mathcal{C}_{Q}^{\prime \prime}=V(\mathbf{f}) \cap \Pi_{e+i-1}^{-1}\left(\mathcal{C}_{Q}^{\prime}\right)$. 
If $\mathcal{C}_{Q}^{\prime}$ is nonempty and finite and if $Q^{\prime}$ is a zero-dimensional parameterization of $\mathcal{C}_{Q}^{\prime}$, it is useful to remark that $\mathcal{C}_{Q}^{\prime \prime}=V\left(\left[\mathbf{f}, Q^{\prime}\right]\right)$. Then, the following theorem summarizes all results we need to ensure the validity of our algorithms.

Theorem 19 If $[\mathbf{f}, Q]$ satisfies $\mathbf{H}$ and $\mathbf{H}_{i}^{\prime}$, then the following holds:

1. $W_{i, Q}$ is either empty or $(i-1)$-equidimensional;

2. $\mathcal{C}_{Q}$ is finite;

3. $\mathcal{C}_{Q}^{\prime \prime}$ is either empty or $(d-i+1)$-equidimensional;

4. $\mathcal{C}_{Q}^{\prime \prime} \cup W_{i, Q}$ is a roadmap of $\left(V([\mathbf{f}, Q]), \mathcal{P}_{Q}\right)$;

5. $\mathcal{C}_{Q}^{\prime \prime} \cap W_{i, Q}=W_{i, Q} \cap \Pi_{e+i-1}^{-1}\left(\mathcal{C}_{Q}^{\prime}\right)$ is finite;

6. if $\mathcal{C}_{Q}^{\prime}$ is not empty and if $Q^{\prime}\left(X_{1}, \ldots, X_{e+i-1}\right)$ is a parameterization of $\mathcal{C}_{Q}^{\prime}$, then [f, $\left.Q^{\prime}\right]$ satisfies assumption $\mathbf{H}$.

Proof This is routine verification. For $\mathbf{x}$ in $Z(Q)$, let $V_{\mathbf{x}}$ be the fiber $V \cap \Pi_{e}^{-1}(\mathbf{x})$, so that $V([\mathbf{f}, Q])$ is the finite union of the algebraic sets $V_{\mathbf{x}}$. Next, we define $w_{i, \mathbf{x}}$ as the set of critical points of $\Pi_{e, i}$ on $V_{\mathbf{x}}$, and similarly $W_{i, \mathbf{x}}, \mathcal{P}_{\mathbf{x}}, \mathcal{C}_{\mathbf{x}}, \mathcal{C}_{\mathbf{x}}^{\prime}$, and $\mathcal{C}_{\mathbf{x}}^{\prime \prime}$ in the obvious manner. One deduces that the disjoint union of the sets $w_{i, \mathbf{x}}$ (resp. $W_{i, \mathbf{x}}, \mathcal{P}_{\mathbf{x}}$, $\mathcal{C}_{\mathbf{x}}, \mathcal{C}_{\mathbf{x}}^{\prime}$, and $\mathcal{C}_{\mathbf{x}}^{\prime \prime}$ ) for $\mathbf{x}$ in $Z(Q)$ is $w_{i, Q}$ (resp. $W_{i, Q}, \mathcal{P}_{Q}, \mathcal{C}_{Q}, \mathcal{C}_{Q}^{\prime}$, and $\mathcal{C}_{Q}^{\prime \prime}$ ).

For $\mathbf{x}$ in $Z(Q)$, let $\tilde{V}_{\mathbf{x}} \subset \mathbf{C}^{n-e}$ be the projection of $V_{\mathbf{x}}$ on the space of coordinates $X_{e+1}, \ldots, X_{n}$ (so that we forget the coordinates $X_{1}, \ldots, X_{e}$ ) and define similarly $\tilde{w}_{i, \mathbf{x}}$, etc. By construction, the system $\mathbf{f}_{\mathbf{x}}$ defines $\tilde{V}_{\mathbf{x}}$; by assumption, it satisfies $\mathbf{H}$ and $\mathbf{H}_{i}^{\prime}$. This allows us to apply Theorem 14; we deduce that in $\mathbf{C}^{n-e}$, we have the following:

- $\tilde{W}_{i, \mathbf{x}}$ is either empty or $(i-1)$-equidimensional;

- $\tilde{\mathcal{C}}_{\mathbf{x}}$ is finite;

- $\tilde{\mathcal{C}}_{\mathbf{x}}^{\prime \prime}$ is either empty or $(d-i+1)$-equidimensional;

- $\tilde{\mathcal{C}}_{\mathbf{x}}^{\prime \prime} \cup \tilde{W}_{i, \mathbf{x}}$ is a roadmap of $\left(\tilde{V}_{\mathbf{x}}, \tilde{\mathcal{P}}_{\mathbf{x}}\right)$;

- $\tilde{\mathcal{C}}_{\mathbf{x}}^{\prime \prime} \cap \tilde{W}_{i, \mathbf{x}}$ is finite;

- for any $\left(x_{e+1}, \ldots, x_{e+i-1}\right)$ in $\mathbf{C}^{i-1}$, the system $\left(f_{1, \mathbf{x}}, \ldots, f_{p, \mathbf{x}}, X_{e+1}-x_{e+1}, \ldots\right.$, $\left.X_{e+i-1}-x_{e+i-1}\right)$ satisfies assumption $\mathbf{H}$.

Back in $\mathbf{C}^{n}$, this translates as follows:

- $W_{i, \mathbf{x}}$ is either empty or $(i-1)$-equidimensional;

- $C_{\mathbf{X}}$ is finite;

- $\mathcal{C}_{\mathrm{x}}^{\prime \prime}$ is either empty or $(d-i+1)$-equidimensional;

- $\mathcal{C}_{\mathbf{x}}^{\prime \prime} \cup W_{i, \mathbf{x}}$ is a roadmap of $\left(V_{\mathbf{x}}, \mathcal{P}_{\mathbf{x}}\right)$;

- $\mathcal{C}_{\mathbf{x}}^{\prime \prime} \cap W_{i, \mathbf{x}}$ is finite;

- for all $\mathbf{x}^{\prime}=\left(x_{1}, \ldots, x_{e+i-1}\right)$ in $\mathbf{C}^{e+i-1}$ such that $\mathbf{x}=\left(x_{1}, \ldots, x_{e}\right)$ is in $Z(Q)$, the system $\left(f_{1, \mathbf{x}}, \ldots, f_{p, \mathbf{x}}, X_{e+1}-x_{e+1}, \ldots, X_{e+i-1}-x_{e+i-1}\right)$ satisfies assumption $\mathbf{H}$.

Taking the union over all $\mathbf{x}$ in $Z(Q)$ proves the theorem.

Corollary 20 Suppose that [f, $Q$ ] satisfies $\mathbf{H}$ and $\mathbf{H}_{i}^{\prime}$, and let $\mathcal{R}_{1}$ and $\mathcal{R}_{2}$ be as follows: 
- $\mathcal{R}_{1}$ is a 1-roadmap of $\left(W_{i, Q},\left(\mathcal{C}_{Q}^{\prime \prime} \cap W_{i, Q}\right) \cup \mathcal{P}_{Q}\right)$;

- $\mathcal{R}_{2}$ is a 1-roadmap of $\left(\mathcal{C}_{Q}^{\prime \prime},\left(\mathcal{C}_{Q}^{\prime \prime} \cap W_{i, Q}\right) \cup \mathcal{P}_{Q}\right)$.

Then, $\mathcal{R}_{1} \cup \mathcal{R}_{2}$ is a 1-roadmap of $\left(V([\mathbf{f}, Q]), \mathcal{P}_{Q}\right)$.

Proof This is a consequence of the former theorem and Proposition 2.

\subsection{Subroutines}

In this subsection, we give a quick overview of the subroutines we use; since most proofs are standard, we postpone them to the end of this section, in Sect. 5.6. Recall that the degree $\delta_{Q}$ associated to a parameterization $Q$ was defined in the introduction.

First, we need a function Union $\left(Q, Q^{\prime}\right)$ that computes a parameterization of the union of two zero-dimensional (resp. one-dimensional) sets, both given by parameterizations.

Lemma 21 Let $Q$ and $Q^{\prime}$ be parameterizations defined over $\mathbf{Q}$. Then one can compute in probabilistic time $\left(n\left(\delta_{Q}+\delta_{Q^{\prime}}\right){ }^{O(1)}\right.$ a parameterization $R$ of $Z(Q) \cup Z\left(Q^{\prime}\right)$ with $\delta_{R} \leq \delta_{Q}+\delta_{Q^{\prime}}$.

Given a zero-dimensional parameterization $Q$, we need a function $\operatorname{Projection}(Q$, $\left.\left[X_{e_{1}}, \ldots, X_{e_{s}}\right]\right)$ that computes a parameterization $R\left(X_{e_{1}}, \ldots, X_{e_{s}}\right)$ such that $Z(R)=$ $\pi(Z(Q))$, where $\pi$ is the projection on the space of coordinates $\left(X_{e_{1}}, \ldots, X_{e_{S}}\right)$.

Lemma 22 Given a zero-dimensional parameterization $Q$ defined over $\mathbf{Q}$, one can compute in probabilistic time $\left(n \delta_{Q}\right)^{O(1)}$ a parameterization $R$ of $\pi(Z(Q))$ with $\delta_{R} \leq \delta_{Q}$.

We will perform linear change of variables on either polynomial systems or parameterizations; the corresponding function will be denoted by ApplyChangeOfVariables in both cases. First, we deal with changing variables in a family of polynomials. Given equations $\mathbf{g}$ and a matrix $\mathbf{A} \in \mathrm{GL}_{n}(\mathbf{Q})$, we want $\tilde{\mathbf{g}}$ such that $V(\tilde{\mathbf{g}})=\varphi(V(\mathbf{g}))$ with $\varphi: \mathbf{x} \mapsto \mathbf{A x}$; this requires us to compose $\mathbf{g}$ by $\mathbf{A}^{-1}$.

Lemma 23 Given $\mathbf{A} \in \mathrm{GL}_{n}(\mathbf{Q})$ and $\mathbf{g}=\left(g_{1}, \ldots, g_{p}\right)$ in $\mathbf{Q}\left[X_{1}, \ldots, X_{n}\right]$ of degree at most $D$, one can compute $\left(\tilde{g}_{1}, \ldots, \tilde{g}_{p}\right)$ with $\tilde{g}_{i}=g_{i}\left(\mathbf{A}^{-1}\left[X_{1} \cdots X_{n}\right]^{t}\right)$ in time $p D^{O(n)}$.

Next, we give the cost of changing variables in a parameterization.

Lemma 24 Given $\mathbf{A} \in \mathrm{GL}_{n}(\mathbf{Q})$ and a parameterization $Q$ defined over $\mathbf{Q}$, one can compute a parameterization $Q^{\prime}$ such that $Z\left(Q^{\prime}\right)=\varphi(Z(Q))$ in time $\left(n \delta_{Q}\right)^{O(1)}$ with $\delta_{Q^{\prime}}=\delta_{Q}$.

We use a function Solve to solve zero- and one-dimensional polynomial systems; more precisely, $\operatorname{Solve}(\mathbf{g}, Q, j)$ will return a parameterization of the $j$-dimensional component of $V([\mathbf{g}, Q])$ with $j \in\{0,1\}$. 
Lemma 25 Consider polynomials $\mathbf{g}=\left(g_{1}, \ldots, g_{t}\right)$ in $\mathbf{Q}\left[X_{1}, \ldots, X_{n}\right]$ of degree at most $D$ and a zero-dimensional parameterization $Q\left(X_{1}, \ldots, X_{e}\right)$ defined over $\mathbf{Q}$. Let $Z=V([\mathbf{g}, Q])$, and let $Z_{j}$ be its $j$-dimensional component for $j \in\{0,1\}$. Then, one can compute in time $\left(t \delta_{Q}\right)^{O(1)} D^{O(n)}$ a parameterization $R$ of $Z_{j}$ with $\delta_{R} \leq \delta_{Q} D^{n}$.

Our variant of Canny's algorithm computes a critical curve (called "silhouette" in [14]). To do so, the function CriticalCurve takes as input polynomials $\mathbf{f} \subset$ $\mathbf{Q}\left[X_{1}, \ldots, X_{n}\right]$ and a zero-dimensional parameterization $Q\left(X_{1}, \ldots, X_{e}\right)$ that satisfy $\mathbf{H}$ and $\mathbf{H}_{2}^{\prime}$. Then, $W_{2, Q}$ is either empty or 1-equidimensional (Theorem 19.1). The function CriticalCurve(f, $Q$ ) computes a parameterization of this algebraic set.

Lemma 26 Consider polynomials $\mathbf{f}=\left(f_{1}, \ldots, f_{p}\right)$ in $\mathbf{Q}\left[X_{1}, \ldots, X_{n}\right]$ of degree at most $D$ and a zero-dimensional parameterization $Q\left(X_{1}, \ldots, X_{e}\right)$ defined over $\mathbf{Q}$. Suppose that $n-e-p \geq 2$ and that [f, Q] satisfies $\mathbf{H}$ and $\mathbf{H}_{2}^{\prime}$. Then, one can compute a parameterization $R$ of $W_{2, Q}$ in probabilistic time $\delta_{Q}^{O(1)}(n D){ }^{O(n)}$ with $\delta_{R} \leq \delta_{Q}(n D)^{n}$.

We also need to compute critical points of a slightly different kind. Consider a system $\mathbf{f}$ and a zero-dimensional parameterization $Q\left(X_{1}, \ldots, X_{e}\right)$ such that $[\mathbf{f}, Q]$ satisfies $\mathbf{H}$ and $\mathbf{H}_{i}^{\prime}$. Then, the algebraic set $\operatorname{crit}\left(\Pi_{e, 1}, V([\mathbf{f}, Q])\right) \cup \operatorname{crit}\left(\Pi_{e, 1}, W_{i, Q}\right)$ is finite (Theorem 19.2). The function RequiredCriticalPoints(f, $Q, i)$ computes a parameterization of this algebraic set.

Lemma 27 Consider polynomials $\mathbf{f}=\left(f_{1}, \ldots, f_{p}\right)$ in $\mathbf{Q}\left[X_{1}, \ldots, X_{n}\right]$ of degree at most $D$ and a zero-dimensional parameterization $Q\left(X_{1}, \ldots, X_{e}\right)$ defined over $\mathbf{Q}$. Suppose that $[\mathbf{f}, Q]$ satisfies $\mathbf{H}$ and $\mathbf{H}_{i}^{\prime}$. Then, one can compute a parameterization $R$ of $\operatorname{crit}\left(\Pi_{e, 1}, V([\mathbf{f}, Q])\right) \cup \operatorname{crit}\left(\Pi_{e, 1}, W_{i, Q}\right)$ in probabilistic time $\delta_{Q}^{O(1)} n^{O(n \min (p, n-e-p))} D^{O(n)}$ with $\delta_{R}=\delta_{Q}(n D){ }^{O(n)}$.

Finally, we need to compute fibers of projections. The first instance of this question is to compute $Z(P) \cap \Pi_{e}^{-1}(Z(Q))$, where $P\left(X_{1}, \ldots, X_{n}\right)$ and $Q\left(X_{1}, \ldots, X_{e}\right)$ are zero-dimensional parameterizations. This will be called $\operatorname{Lift}(P, Q)$.

Lemma 28 Let $P\left(X_{1}, \ldots, X_{n}\right)$ and $Q\left(X_{1}, \ldots, X_{e}\right)$ be zero-dimensional parameterizations defined over $\mathbf{Q}$. Then one can compute in probabilistic time $\left(n\left(\delta_{P}+\delta_{Q}\right)\right) O(1)$ a parameterization $R$ of $Z(P) \cap \Pi_{e}^{-1}(Z(Q))$ with $\delta_{R} \leq \delta_{P}$.

The second form of this question is more complex. To apply Theorem 19, we need to compute $\mathcal{C}_{Q}^{\prime \prime} \cap W_{i, Q}=W_{i, Q} \cap \Pi_{e+i-1}^{-1}\left(Z\left(Q^{\prime}\right)\right)$, where $\mathbf{f}$ and $Q$ satisfy $\mathbf{H}$ and $\mathbf{H}_{i}^{\prime}$, and $Q^{\prime}\left(X_{1}, \ldots, X_{e+i-1}\right)$ is a zero-dimensional parameterization of $\mathcal{C}_{Q}^{\prime}$. By Theorem 19.5, $W_{i, Q} \cap \Pi_{e+i-1}^{-1}\left(Z\left(Q^{\prime}\right)\right)$ is finite. The function LiftW(f, $\left.Q^{\prime}\right)$ returns a zero-dimensional (or empty) parameterization $R$ such that $Z(R)$ contains this algebraic set.

Lemma 29 Consider polynomials $\mathbf{f}=\left(f_{1}, \ldots, f_{p}\right)$ in $\mathbf{Q}\left[X_{1}, \ldots, X_{n}\right]$, of degree at most $D$ and a zero-dimensional parameterization $Q\left(X_{1}, \ldots, X_{e}\right)$ defined over $\mathbf{Q}$ 
that satisfy $\mathbf{H}$ and $\mathbf{H}_{i}^{\prime}$. Let further $Q^{\prime}\left(X_{1}, \ldots, X_{e+i-1}\right)$ be a zero-dimensional parameterization of $\mathcal{C}_{Q}^{\prime}$. Then one can compute in probabilistic time $\delta_{Q^{\prime}}^{O(1)}(n D){ }^{O(n)}$ a zero-dimensional (or empty) parameterization $R$ such that $W_{i, Q} \cap \Pi_{e+i-1}^{-1}\left(Z\left(Q^{\prime}\right)\right)$ is contained in $Z(R)$ and with $\delta_{R}=\delta_{Q^{\prime}}(n D){ }^{O(n)}$.

\subsection{Canny's Algorithm Revisited}

We give in this section an algorithm close to Canny's. We take as input a system $\mathbf{f}=\left(f_{1}, \ldots, f_{p}\right)$ in $\mathbf{Q}\left[X_{1}, \ldots, X_{n}\right]$ of degree at most $D \geq 2$. We also consider as input a zero-dimensional parameterization $Q\left(X_{1}, \ldots, X_{e}\right)$ defined over $\mathbf{Q}$; writing $d=n-p-e$, we assume that $d>0$ and that [f, $Q]$ that satisfies $\mathbf{H}$. Our last input are control points $\mathcal{P}$ given in the form of a zero-dimensional parameterization $P$.

If $d=1$, we are done: it suffices to solve the system. Else, as Canny, we take $i=2$ in the recursion. Indeed, given such a system, we will see that it is possible to ensure assumption $\mathbf{H}_{2}^{\prime}$ through a generic change of variables; higher values of $i$ may not allow us to ensure this assumption. Our change of variables will leave $X_{1}, \ldots, X_{e}$ fixed; we denote by $\mathrm{GL}(n, e)$ the subset of $\mathrm{GL}_{n}(\mathbf{Q})$ satisfying this constraint.

CannyRoadmap $(\mathbf{f}, Q, P)$.

1. If $n-p-e=1$, return Solve $(\mathbf{f}, Q, 1)$

2. Let $\mathbf{A}$ be a random matrix in $\operatorname{GL}(n, e)$

3. Let $\mathbf{f}=$ ApplyChangeOfVariables $(\mathbf{A}, \mathbf{f})$

4. Let $P=$ ApplyChangeOfVariables $(\mathbf{A}, P)$

5. Let $P=\operatorname{Lift}(P, Q)$

6. Let $C=$ Union(RequiredCriticalPoints $(\mathbf{f}, Q, 2), P$ )

7. Let $Q^{\prime}=\operatorname{Projection}\left(C,\left[X_{1}, \ldots, X_{e+1}\right]\right)$

8. Let $P^{\prime}=\operatorname{Union}\left(\operatorname{LiftW}\left(\mathbf{f}, Q^{\prime}\right), P\right)$

9. If $C$ is different from $(-1)$, then $Z\left(P^{\prime}\right)$ contains $\left(\mathcal{C}_{Q}^{\prime \prime} \cap W_{2, Q}\right) \cup \mathcal{P}_{Q}$

let $R=$ CannyRoadmap $\left(\mathbf{f}, Q^{\prime}, P^{\prime}\right) \quad V\left(\left[\mathbf{f}, Q^{\prime}\right]\right)=\mathcal{C}_{Q}^{\prime \prime}$, and $e$ increases by 1 else

let $R=(-1)$

$\mathcal{C}$ is empty

10. Let $R^{\prime}=$ CriticalCurve $(\mathbf{f}, Q)$

11. Let $R^{\prime \prime}=\operatorname{Union}\left(R, R^{\prime}\right)$ $Z\left(R^{\prime}\right)=W_{2, Q}$

12. Return ApplyChangeOfVariables $\left(\mathbf{A}^{-1}, R^{\prime \prime}\right)$

Proposition 30 Suppose that [f, Q] satisfies assumption $\mathbf{H}$. Then, algorithm CannyRoadmap outputs a 1-roadmap of $(V([\mathbf{f}, Q]), \mathcal{P})$ of degree $\left(\delta_{Q}+\delta_{P}\right) \times$ $(n D)^{O(n(n-p-e))}$ in probabilistic time $\left(\delta_{Q}+\delta_{P}\right)^{O(1)}(n D) O(n(n-p-e))$.

The remainder of this subsection is devoted to prove this proposition. The first ingredient of the proof is the following genericity argument; it is proved in Sect. 6.

Lemma 31 Suppose that $n-p-e>1$ and that $[\mathbf{f}, Q]$ satisfies $\mathbf{H}$. After a generic change of variables in $\mathrm{GL}(n, e)$, the system $[\mathbf{f}, Q]$ satisfies $\mathbf{H}_{2}^{\prime}$ as well. 
The following lemma gives complexity estimates for most steps; we exclude the last steps, since handling them would require unrolling the recurrence giving degree bounds along all levels of the recursion.

Lemma 32 Suppose that $n-p-e>1$, that $[\mathbf{f}, Q]$ satisfies $\mathbf{H}$, and that after the change of variables $\mathbf{A}$, [f, $Q$ ] satisfies $\mathbf{H}_{2}^{\prime}$. Then, Steps 3-8 and 10 of algorithm CannyRoadmap take probabilistic time $\left(\delta_{Q}+\delta_{P}\right)^{O(1)} n^{O(n(n-p-e))} D^{O(n)}$. Upon success, $Q^{\prime}, P^{\prime}$ are zero-dimensional (or empty) parameterizations, and $R^{\prime}$ is a onedimensional (or empty) parameterization that satisfy

$$
\delta_{Q^{\prime}}+\delta_{P^{\prime}}=\left(\delta_{Q}+\delta_{P}\right)(n D)^{O(n)}, \quad \delta_{R^{\prime}}=\delta_{Q}(n D)^{O(n)},
$$

and if $C$ is not empty, $\left[\mathbf{f}, Q^{\prime}\right]$ satisfies $\mathbf{H}$. If the inner call at Step 9 computes a 1-roadmap of $\left(V\left(\left[\mathbf{f}, Q^{\prime}\right]\right), Z\left(P^{\prime}\right)\right)$, then the output at Step 12 is a 1-roadmap of $(V([\mathbf{f}, Q]), \mathcal{P})$.

Proof We start by proving correctness. At Step 5, $P$ is such that $Z(P)=\mathcal{P}_{Q}$. At Step 6, $C$ is a parameterization of $\mathcal{C}_{Q}$, either empty or zero-dimensional (Theorem 19.2). At Step 7, $Z\left(Q^{\prime}\right)$ is the projection $\mathcal{C}_{Q}^{\prime}$, whence in particular $V\left(\left[\mathbf{f}, Q^{\prime}\right]\right)=$ $\mathcal{C}_{Q}^{\prime \prime}$. At Step 8, $Z\left(P^{\prime}\right)$ is finite (Theorem 19.5) and contains the new set of control points $\left(\mathcal{C}_{Q}^{\prime \prime} \cap W_{2, Q}\right) \cup \mathcal{P}_{Q}$. At Step 9 , if $\mathcal{C}_{Q}$ is not empty, $\mathcal{C}_{Q}^{\prime}$ is not empty, and [f, $Q^{\prime}$ ] satisfies $\mathbf{H}$ (Theorem 19.6); in this case, this justifies the recursive call. In both cases, the output $Z(R)$ is a 1-roadmap of $\left(\mathcal{C}_{Q}^{\prime \prime}, Z\left(P^{\prime}\right)\right)$ and thus of $\left(\mathcal{C}_{Q}^{\prime \prime},\left(\mathcal{C}_{Q}^{\prime \prime} \cap W_{2, Q}\right) \cup \mathcal{P}_{Q}\right)$.

Theorem 19.1 implies that at Step $10, Z\left(R^{\prime}\right)$ equals $W_{2, Q}$ (it may be empty); remark that $W_{2, Q}$ is tautologically a 1-roadmap of $\left.\left(W_{2, Q}, \mathcal{C}_{Q}^{\prime \prime} \cap W_{2, Q}\right) \cup \mathcal{P}_{Q}\right)$. Then, Corollary 20 shows that $Z\left(R^{\prime \prime}\right)=Z(R) \cup Z\left(R^{\prime}\right)=\mathcal{C}_{Q}^{\prime \prime} \cup W_{2, Q}$ is a 1-roadmap of $\left(V([\mathbf{f}, Q]), \mathcal{P}_{Q}\right)$. This establishes correctness.

Next, we estimate the running time of the first steps and give degree bounds, assuming correctness. Lemmas 23 and 24 show that applying $\mathbf{A}$ takes time $\left(n \delta_{P}\right)^{O(1)}+$ $n D^{O(n)}$; in the new variables, the degrees $D$ of $\mathbf{f}$ and $\delta_{P}$ of $P$ are unchanged. By Lemma 28 , the cost of Step 5 is $\left(n\left(\delta_{Q}+\delta_{P}\right)\right)^{O(1)}$, and $\delta_{P}$ can only decrease through this process.

By Lemmas 27 and 21, $C$ can be computed in probabilistic time $\delta_{Q}^{O(1)} \times$ $n^{O(n(n-p-e))} D^{O(n)}+\left(n \delta_{P}\right)^{O(1)}$, and $\delta_{C}$ is bounded by $\delta_{Q}(n D) O(n)+\delta_{P}$. By Lemma 22, $Q^{\prime}$ can be computed in probabilistic time $\delta_{Q}^{O(1)}(n D){ }^{O(n)}+\left(n \delta_{P}\right)^{O(1)}$, and we have $\delta_{Q^{\prime}}=\delta_{Q}(n D){ }^{O(n)}+\delta_{P}$. Thus, in view of Lemmas 29 and $21, P^{\prime}$ satisfies $\delta_{P^{\prime}}=\left(\delta_{Q}+\delta_{P}\right)(n D)^{O(n)}$ and can be computed in time $\left(\delta_{Q}+\delta_{P}\right)^{O(1)}(n D) O(n)$. The degree and time bounds on $R^{\prime}$ follow from Lemma 26.

We now prove Proposition 30. Correctness follows from the previous lemma, so we focus on degree bounds and runtime, assuming that all changes of variables are lucky. Let us rename the input $(Q, P)$ as $\left(Q_{0}, P_{0}\right)$. The number of recursive calls is $n-p-e$, and Lemma 32 shows that each recursive call multiplies $\delta_{Q}+\delta_{P}$ by $(n D)^{O(n)}$, so that $\delta_{Q}+\delta_{P}=\left(\delta_{Q_{0}}+\delta_{P_{0}}\right)(n D){ }^{O(n(n-p-e))}$ holds at all levels. The output of CannyRoadmap is the union of the critical curves computed at Steps 10 
of the recursive levels, and all of them have degree $\left(\delta_{Q_{0}}+\delta_{P_{0}}\right)(n D) O(n(n-p-e))$ by Lemma 32. Since there are $O(n)$ such curves, the degree bound on the output follows.

The runtime estimate follows similarly from the previous lemma. The sum of the costs involved in Steps 3-8 and 10 (including all levels of the recursion) fits into the claimed bound, in view on the former estimate on $\delta_{Q}+\delta_{P}$; the same holds for the cost at Step 1. All that is missing is the cost of Steps 11 and 12; using the previous degree estimate on $Z(R)$, it follows from Lemmas 21 and 24.

\subsection{Main Subroutine}

We give now our roadmap algorithm for a hypersurface $V(f)$, where $f \in \mathbf{Q}\left[X_{1}\right.$, $\left.\ldots, X_{n}\right]$ satisfies assumption $\mathbf{H}$ and has degree $D \geq 2$. Here, we can ensure assumption $\mathbf{H}_{i}^{\prime}$ in generic coordinates for many more choices of $i$. Using our modified version of Canny's algorithm as a subroutine, our strategy takes $i \simeq \sqrt{n}$ : this will balance the cost of the main function and that of Canny's algorithm. As before, we also take a zero-dimensional parameterization $Q\left(X_{1}, \ldots, X_{e}\right)$ over $\mathbf{Q}$ as input and the control points $\mathcal{P}$ by means of a zero-dimensional parameterization $P$.

MainRoadmap $(f, Q, P, i)$.

1. If $n-e \leq i$, return CannyRoadmap $(f, Q)$

2. Let $\mathbf{A}$ be a random matrix in $\operatorname{GL}(n, e)$

3. Let $f=$ ApplyChangeOfVariables $(\mathbf{A}, f)$

4. Let $P=$ ApplyChangeOfVariables $(\mathbf{A}, P)$

5. Let $P=\operatorname{Lift}(P, Q)$

6. Let $C=$ Union(RequiredCriticalPoints $(f, Q, i), P)$

$Z(P)=\mathcal{P}_{Q}$

7. Let $Q^{\prime}=\operatorname{Projection}\left(C,\left[X_{1}, \ldots, X_{e+i-1}\right]\right)$

$Z(C)=\mathcal{C}_{Q}$

8. Let $P^{\prime}=\operatorname{Union}\left(\operatorname{LiftW}\left(f, Q^{\prime}\right), P\right)$

9. If $C$ is different from $(-1)$, then $Z\left(Q^{\prime}\right)=\mathcal{C}_{Q}^{\prime}$

Let $R=$ MainRoadmap $\left(f, Q^{\prime}, P^{\prime}, i\right) \quad V\left(\left[f, Q^{\prime}\right]\right)=\mathcal{C}_{Q}^{\prime \prime}$ and $e$ increases by $i$ else

let $R=(-1)$

C is empty

10. Let $\Delta=\left[\partial f / \partial X_{i} \mid i \in[e+i+1, \ldots, n]\right]$ and let $\mathbf{f}=(f, \Delta)$

11. Let $R^{\prime}=$ CannyRoadmap $\left(\mathbf{f}, Q, P^{\prime}\right)$

12. Let $R^{\prime \prime}=\operatorname{Union}\left(R, R^{\prime}\right)$

13. Return ApplyChangeOfVariables $\left(\mathbf{A}^{-1}, R^{\prime \prime}\right)$

Proposition 33 Let $j=(n-e) / i$ and suppose that $[f, Q]$ satisfies assumption $\mathbf{H}$. Then algorithm MainRoadmap outputs a 1-roadmap of $(V([f, Q]), \mathcal{P})$ of degree $\left(\delta_{Q}+\delta_{P}\right)(n D)^{O(n(i+j))}$ in probabilistic time $\left(\delta_{Q}+\delta_{P}\right)^{O(1)}(n D)^{O(n(i+j))}$.

Remark that taking $e=0$ and $i=\lfloor\sqrt{n}\rfloor$ proves Theorem 1 . The rest of this subsection is devoted to prove this result; in all that follows, remember that the parameter $i$ is fixed. The proof is similar to that of our modified version of Canny's algorithm. As before, a key ingredient is a genericity argument whose proof is given in Sect. 6. 
Lemma 34 Suppose that $n-e>i$ and that $[f, Q]$ satisfies $\mathbf{H}$. After a generic change of variables in $\operatorname{GL}(n, e)$,

(a) $[f, Q]$ satisfies $\mathbf{H}$ and $\mathbf{H}_{i}^{\prime}$;

(b) [f, Q] satisfies $\mathbf{H}$.

Proposition 30 shows that the cost of the case $n-e \leq i$ is $\left(\delta_{P}+\delta_{Q}\right)^{O(1)}(n D){ }^{O(n i)}$. Assuming that we are not in this base case, the following lemma gives complexity estimates for the first steps of MainRoadmap. As for CannyRoadmap, we exclude the cost of the last steps for the moment.

Lemma 35 Suppose that $n-e>i$, that $[f, Q]$ satisfies $\mathbf{H}$, and that after the change of variables $\mathbf{A},[f, Q]$ satisfies $\mathbf{H}_{i}^{\prime}$, and $[\mathbf{f}, Q]$ satisfies $\mathbf{H}$. Then, Steps 3-8 and 10, 11 of algorithm MainRoadmap take probabilistic time $\left(\delta_{Q}+\delta_{P}\right)^{O(1)}(n D)^{O(n i)}$; upon success, $Q^{\prime}, P^{\prime}$ are zero-dimensional (or empty) parameterizations, and $R$ is a onedimensional (or empty) parameterization that satisfy

$$
\delta_{Q^{\prime}}+\delta_{P^{\prime}}=\left(\delta_{Q}+\delta_{P}\right)(n D)^{O(n)}, \quad \delta_{R}=\left(\delta_{Q}+\delta_{P}\right)(n D)^{O(n i)},
$$

and if $C$ is not empty, $\left[f, Q^{\prime}\right]$ satisfies assumption $\mathbf{H}$. If the inner calls at Steps 9 and 11 are successful, then the output at Step 13 is a roadmap of $(V([f, Q]), \mathcal{P})$.

Proof We start by proving correctness. The proof follows the same pattern as that of Lemma 32.

At Step 5, we have $Z(P)=\mathcal{P}_{Q}$. At Step 6,C is parameterization such that $Z(C)=\mathcal{C}_{Q}$, either empty or zero-dimensional (Theorem 19.2). At Step 7, $Z\left(Q^{\prime}\right)$ equals $\mathcal{C}_{Q}^{\prime}$, so $V\left(\left[f, Q^{\prime}\right]\right)=\mathcal{C}_{Q}^{\prime \prime}$. At Step $8, Z\left(P^{\prime}\right)$ is finite (Theorem 19.5) and contains the new set of control points $\left(\mathcal{C}_{Q}^{\prime \prime} \cap W_{i, Q}\right) \cup \mathcal{P}_{Q}$. At Step 9 , if $\mathcal{C}_{Q}$ is not empty, $\mathcal{C}_{Q}^{\prime}$ and $\left[f, Q^{\prime}\right]$ satisfy $\mathbf{H}$ (Theorem 19.6); this justifies the recursive call. In both cases, the output $Z(R)$ is a 1-roadmap of $\left(\mathcal{C}_{Q}^{\prime \prime}, Z\left(P^{\prime}\right)\right)$ and thus of $\left(\mathcal{C}_{Q}^{\prime \prime},\left(\mathcal{C}_{Q}^{\prime \prime} \cap W_{i, Q}\right) \cup \mathcal{P}_{Q}\right)$.

At Step 11 , let us justify that $V([\mathbf{f}, Q])=W_{i, Q}$. By construction, this system defines $\operatorname{crit}\left(\Pi_{e, i}, V([f, Q])\right)$, which equals $W_{i, Q} \cup \operatorname{sing}(V([f, Q]))$. By Krull's theorem, there are no isolated points in $V([\mathbf{f}, Q])$; since $\operatorname{sing}(V([f, Q]))$ is finite, it is actually included in $W_{i, Q}$, and our claim follows. Consequently, $Z\left(R^{\prime}\right)$ is a 1-roadmap of $\left(W_{i, Q}, Z\left(P^{\prime}\right)\right)$ and thus of $\left(W_{i, Q},\left(\mathcal{C}_{Q}^{\prime \prime} \cap W_{i, Q}\right) \cup \mathcal{P}_{Q}\right)$. Then, Corollary 20 shows that $Z\left(R^{\prime \prime}\right)=Z(R) \cup Z\left(R^{\prime}\right)$ is a 1-roadmap of $\left(V([f, Q]), \mathcal{P}_{Q}\right)$. This establishes correctness.

Next, we estimate the running time and the degree of the output, assuming correctness. Lemmas 23 and 24 shows that applying $\mathbf{A}$ takes time $\left(n \delta_{P}\right) O(1)+D^{O(n)}$. By Lemma 28 , the cost of Step 5 is $\left(n\left(\delta_{Q}+\delta_{P}\right)\right)^{O(1)}$, and $\delta_{P}$ can only decrease through this process. By Lemmas 27 and 21, $C$ can be computed in probabilistic time $\delta_{Q}^{O(1)}(n D)^{O(n)}+\left(n \delta_{P}\right)^{O(1)}$, and $\delta_{C}$ is bounded by $\delta_{Q}(n D)^{n}+\delta_{P}$. By Lemma 22, $Q^{\prime}$ can be computed in probabilistic time $\delta_{Q}^{O(1)}(n D)^{O(n)}$, and we have $\delta_{Q^{\prime}} \leq \delta_{Q}(n D)^{n}+\delta_{P}$. Thus, by Lemma $29, P^{\prime}$ satisfies $\delta_{P^{\prime}}=\left(\delta_{Q}+\delta_{P}\right)(n D){ }^{O(n)}$ and can be computed in time $\left(\delta_{Q}+\delta_{P}\right)^{O(1)}(n D) O(n)$. 
The call to CannyRoadmap has $p=n-e-i+1$ and uses the same specialization values $Q$ of $\left(X_{1}, \ldots, X_{e}\right)$. Thus, Proposition 30 shows that it takes probabilistic time $\left(\delta_{Q}+\delta_{P}\right)^{O(1)}(n D) O(n i)$ and that, upon success, $R^{\prime}$ satisfies $\delta_{R^{\prime}}=$ $\left(\delta_{Q}+\delta_{P}\right)(n D) O(n i)$.

We prove now Proposition 33. Correctness follows from Proposition 30 and from the previous lemma. We now prove degree bounds and runtime, assuming correctness; as before, we let $Q_{0}$ and $P_{0}$ denote our input.

The number of recursive calls is $O((n-e) / i)=O(j)$, and Lemma 35 shows that each recursive call multiplies $\delta_{Q}+\delta_{P}$ by $(n D)^{O(n)}$, so that $\delta_{Q}+\delta_{P}=$ $\left(\delta_{Q_{0}}+\delta_{P_{0}}\right)(n D)^{O(n j)}$ holds at all levels. As a first consequence, the cost of the base case is $\left(\delta_{Q_{0}}+\delta_{P_{0}}\right)^{O(1)}(n D){ }^{O(n(i+j))}$, and the output of the base case has degree $\left(\delta_{Q_{0}}+\delta_{P_{0}}\right)^{O(1)}(n D){ }^{O(n(i+j))}$.

Still using Lemma 35, we deduce that the total cost of Steps 3-11 (counting all recursive calls) is $\left(\delta_{Q_{0}}+\delta_{P_{0}}\right)^{O(1)}(n D) O(n(i+j))$. Besides, the same lemma also shows that all degrees $\delta_{R}$ are bounded by $\left(\delta_{Q_{0}}+\delta_{P_{0}}\right)(n D) O(n(i+j))$ as well. The union operation at Step 12, and the final change of variables induces another cost in $\left(\delta_{Q_{0}}+\delta_{P_{0}}\right)^{O(1)}(n D)^{O(n(i+j))}$.

\subsection{Proofs of the Subroutines}

Finally, we give more details on how to implement the subroutines described previously. Many results are either well known or close to well-known ones; then, we shall be rather sketchy.

Union (Proof of Lemma 21) Consider two parameterizations $Q$ and $Q^{\prime}$, either both zero- or both one-dimensional; we want to compute $R$ such that $Z(R)=Z(Q) \cup$ $Z\left(Q^{\prime}\right)$. We start with degree estimates: by definition, the degree $\delta_{R}=Z(R)$ is at $\operatorname{most} \delta_{Q}+\delta_{Q^{\prime}}$.

We first show how to compute $R$ if we are in dimension zero. We will find a primitive element of $Z(Q) \cup Z\left(Q^{\prime}\right)$ by trying successive candidates $\tau$ : by [32, Lemma 2.1], it suffices to try $\left(n\left(\delta_{Q}+\delta_{Q^{\prime}}\right)\right) O(1)$ candidates. For each candidate $\tau$, we use the algorithm of [25, Lemma 6] to make $\tau$ the primitive element of $Q$ and $Q^{\prime}$ : if this is not possible, we dismiss $\tau$.

Next, writing $Q=\left(q, q_{1}, \ldots, q_{n}\right)$ and $Q^{\prime}=\left(q^{\prime}, q_{1}^{\prime}, \ldots, q_{n}^{\prime}\right)$, we compute $g=$ $\operatorname{gcd}\left(q, q^{\prime}\right), \tilde{q}=q / g$ and $\tilde{q}^{\prime}=q^{\prime} / g$. If $q_{i} \bmod g \neq q_{i}^{\prime} \bmod g$ for some $i$, then $\tau$ is not primitive for $Z(Q) \cup Z\left(Q^{\prime}\right)$. Else, the new minimal polynomial is $q^{\prime \prime}=g \tilde{q} \tilde{q}^{\prime}$, and we deduce parameterizations using the Chinese Remainder Theorem. The running time is polynomial in $n\left(\delta_{Q}+\delta_{Q^{\prime}}\right)$.

In positive dimension, the approach is similar. We first find a linear form $\eta$ suitable for both $Q$ and $Q^{\prime}$ : the only condition is that $\eta$ should take an infinite number of values on both $Z(Q)$ and $Z\left(Q^{\prime}\right)$. Then, we proceed to find $\tau$ as above, using evaluation and interpolation to avoid handling rational functions in the variable $\eta$ through the computations. Again, the running time is polynomial in $n\left(\delta_{Q}+\delta_{Q^{\prime}}\right)$. 
Projection (Proof of Lemma 22) Given a zero-dimensional parameterization $Q$, let us suppose (for simplicity) that we want to compute the projection of $\pi(Z(Q))$ on the space of coordinates $e_{1}=1, \ldots, e_{s}=s$, by means of a parameterization $R$.

Degree estimates are straightforward. To compute $R$, as before, we examine candidate primitive elements for it. For any of them, say $\mu=\mu_{1} X_{1}+\cdots+\mu_{s} X_{s}$, we compute a Gröbner basis of the ideal generated by

$$
q(T), X_{1}-q_{1}(T), \ldots, X_{n}-q_{n}(T), S-\mu_{1} X_{1}-\cdots-\mu_{s} X_{s}
$$

for the order $T>X_{n}>\cdots>X_{1}>S$. If $\mu$ is primitive for $\pi(Z(Q))$, one can read the required parameterization on the last $s+1$ polynomials of the basis. The conversion can be done by e.g. the FGLM algorithm [20], so the total time is $\left(n \delta_{Q}\right)^{O(1)}$.

Change of Variables (Proof of Lemmas 23 and 24) The easier question is to apply a change of variables matrix $\mathbf{A} \in \mathrm{GL}_{n}(\mathbb{Q})$ to a polynomial $f \in \mathbf{Q}\left[X_{1}, \ldots, X_{n}\right]$ of degree $D$ : computing successively all powers of the linear forms $\mathbf{A}^{-1} \cdot X_{1}, \ldots, \mathbf{A}^{-1}$. $X_{n}$ and combining them have cost polynomial in $D^{n}$.

Next, we explain how change of variables operate on a parameterization $Q$. Degree bounds are obvious, since changes of variables do not affect the geometric degree of $Z(Q)$. The input parameterization $Q$ consists in $\left(q, q_{0}, q_{1}, \ldots, q_{n}\right) \subset \mathbf{Q}[T]$. Then, computing a parameterization of $\varphi(Z(Q))$ with $\varphi: \mathbf{x} \mapsto \mathbf{A x}$ is simply done by multiplying $\mathbf{A}$ by the vector $\left[q_{1}, \ldots, q_{n}\right]$, so the running time is $\left(n \delta_{Q}\right)^{O(1)}$.

Solving Systems (Proof of Lemma 25) Given a system $\mathbf{g}=\left(g_{1}, \ldots, g_{t}\right)$ and a zerodimensional parameterization $Q\left(X_{1}, \ldots, X_{e}\right)$, we describe how to solve the system $\left\{g_{1}(\mathbf{x})=\cdots=g_{t}(\mathbf{x})=0, \Pi_{e}(\mathbf{x}) \in Z(Q)\right\}$. We let $W$ be the set of solutions and explain how to compute the zero- or one-dimensional component of $W$.

We start with degree estimates. Since $W=Z(Q) \cap V(\mathbf{g})$, we deduce from the Bézout inequality given in [28, Proposition 2.3] that $\operatorname{deg}(W) \leq \operatorname{deg}(Z(Q)) D^{\operatorname{dim}(Z(Q))}$, which is bounded by $\delta_{Q} D^{n}$. If $R$ is a parameterization of $W$, we deduce by definition that $\delta_{R} \leq \delta_{Q} D^{n}$.

We now consider runtime. If we only had to take $\mathbf{g}$ into account, it would suffice to apply the algorithm of [29] to obtain a cost $t^{O(1)} D^{O(n)}$. However, we also need to take $Q$ into account, and this induces extra complications; we cannot directly append it to our system, since the resulting cost would exceed our target.

We solve this issue using dynamic evaluation techniques [17]. Let $Q$ be given by polynomials $q, q_{1}, \ldots, q_{e}$ in $\mathbf{Q}[T]$, and let $\tau$ be its primitive element. We apply the algorithm of [29] in $\mathbf{L}\left[X_{e+1}, \ldots, X_{n}\right]$ with $\mathbf{L}=\mathbf{Q}[T] / q$, replacing $X_{1}, \ldots, X_{e}$ by $q_{1}, \ldots, q_{e}$. $\mathbf{L}$ is not a field but a product of fields; if a division by a zero-divisor occurs, we split $q$ into two factors, and we run the computation again. The maximal number of splittings is $\delta_{Q}$, and the cost of computing modulo a factor of $q$ is $\delta_{Q}^{O(1)}$. Then, the overall cost is now $\left(t \delta_{Q}\right)^{O(1)} D^{O(n)}$.

At this stage, our output consists in a collection of zero-dimensional or onedimensional parameterizations $R_{i}\left(X_{e+1}, \ldots, X_{n}\right)$ for $i=1, \ldots, s$; they are defined over various products of fields $\mathbf{L}_{i}=\mathbf{Q}[T] / q^{(i)}$, where $q^{(i)}$ are factors of $q$. To conclude, we must first define them over $\mathbf{Q}$. Suppose for definiteness that we are 
in dimension one (dimension zero is simpler); then, $R_{i}$ is given by polynomials $r^{(i)}, r_{0}^{(i)}, r_{e+1}^{(i)}, \ldots, r_{n}^{(i)}$ with $r^{(i)}$ and all $r_{j}^{(i)}$ in $\mathbf{L}_{i}\left[U^{\prime}, T^{\prime}\right]$; the degrees in $\left(U^{\prime}, T^{\prime}\right)$ of these polynomials are all $D^{O(n)}$. One convenient way to obtain a parameterization defined over $\mathbf{Q}$ is to call once more the algorithm of [29] with input the trivariate system $q^{(i)}(T)$ and $r^{(i)}\left(U^{\prime}, T^{\prime}\right)$. Solving one such system takes time polynomial in $n \operatorname{deg}\left(q^{(i)}\right) D^{n}$, so that the total time is $\left(n \delta_{Q}\right)^{O(1)} D^{O(n)}$; from this we obtain parameterizations $R_{i}^{\prime}\left(X_{1}, \ldots, X_{n}\right)$ defined over $\mathbf{Q}$, whose union describes the onedimensional component of $W$. To conclude, it suffices to repeatedly call the union algorithm; the cost estimate is similar.

Computing Critical Curves (Proof of Lemma 26) Next, we compute the polar variety $W_{2, Q}$ associated to a system $\mathbf{f}=\left(f_{1}, \ldots, f_{p}\right)$ and a parameterization $Q\left(X_{1}, \ldots, X_{e}\right)$. Denote by $\Delta$ the set of $p$-minors of $\operatorname{jac}\left(\mathbf{f},\left[X_{e+3}, \ldots, X_{n}\right]\right)$. It contains $\left(\begin{array}{c}n-e-2 \\ p\end{array}\right) \leq 2^{n}$ polynomials of degree bounded by $n D$. Observe that $V([(\mathbf{f}, \Delta), Q])$ is the critical locus $W_{2, Q} \cup \operatorname{sing}(V([\mathbf{f}, Q]))$. By $\mathbf{H}$ and $\mathbf{H}_{2}^{\prime}, W_{2, Q}$ is either empty or purely one-dimensional; on the other hand, $\operatorname{sing}(V([\mathbf{f}, Q]))$ is finite. It follows that the one-dimensional component of $V([(\mathbf{f}, \Delta), Q])$ is $W_{2, Q}$. Thus, Solve $((\mathbf{f}, \Delta), Q, 1)$ returns a one-dimensional parameterization $R$ that describes $W_{2, Q}$ in probabilistic time $\delta_{Q}^{O(1)}(n D){ }^{O(n)}$ with $\delta_{R} \leq \delta_{Q}(n D)^{n}$.

Computing Required Critical Points (Proof of Lemma 27) With the same notation as above, we continue with the computation of the critical points $\operatorname{crit}\left(\Pi_{e, 1}, V([\mathbf{f}, Q])\right)$ and $\operatorname{crit}\left(\Pi_{e, 1}, W_{i, Q}\right)$. To do so, we define several families of determinants:

- $\Delta$ is the set of $p$-minors of $\operatorname{jac}\left(\mathbf{f},\left[X_{e+2}, \ldots, X_{n}\right]\right)$;

- $\Delta^{\prime}$ is the set of $p$-minors of $\operatorname{jac}\left(\mathbf{f},\left[X_{e+i+1}, \ldots, X_{n}\right]\right)$;

- $\Delta^{\prime \prime}$ is the set of $(n-e-i+1)$-minors of jac $\left(\left(\mathbf{f}, \Delta^{\prime}\right),\left[X_{e+2}, \ldots, X_{n}\right]\right)$.

The degree of the polynomials in $\Delta$ and $\Delta^{\prime}$ is at most $n D$; that of the polynomials in $\Delta^{\prime \prime}$ is at most $n^{2} D$. Besides, $\Delta$ has cardinality at most $\left(\begin{array}{c}n-e-1 \\ p\end{array}\right) \leq 2^{n}$, and $\Delta^{\prime}$ has cardinality at most $\left(\begin{array}{c}n-e-i \\ p\end{array}\right) \leq n^{\min (p, n-e-p)}$. Consequently, $\Delta^{\prime \prime}$ contains $n^{O(n \min (p, n-e-p))}$ polynomials. The following lemma shows which system is to be solved to answer our question.

\section{Lemma 36 The equality}

$$
V([(\mathbf{f}, \Delta), Q]) \cup V\left(\left[\left(\mathbf{f}, \Delta^{\prime}, \Delta^{\prime \prime}\right), Q\right]\right)=\operatorname{crit}\left(\Pi_{e, 1}, V([\mathbf{f}, Q])\right) \cup \operatorname{crit}\left(\Pi_{e, 1}, W_{i, Q}\right)
$$

holds.

Proof Since [f, $Q]$ satisfies $\mathbf{H}$ and $\mathbf{H}_{i}^{\prime}$, the following holds:

1. $\operatorname{crit}\left(\Pi_{e, 1}, V([\mathbf{f}, Q])\right)=V([(\mathbf{f}, \Delta), Q])$;

2. $\operatorname{crit}\left(\Pi_{e, i}, V([\mathbf{f}, Q])\right)=V\left(\left[\left(\mathbf{f}, \Delta^{\prime}\right), Q\right]\right)$;

3. $\operatorname{crit}\left(\Pi_{e, i}, V([\mathbf{f}, Q])\right)=W_{i, Q} \cup \operatorname{sing}(V([\mathbf{f}, Q]))$;

4. $W_{i, Q}$ is either empty or $(i-1)$-equidimensional; 
5. Let $\mathbf{x}^{\prime}=\left(x_{1}, \ldots, x_{e}\right)$ be in $Z(Q)$, let $\mathbf{x}=\left(x_{1}, \ldots, x_{n}\right)$ be in $W_{i, Q}-\operatorname{sing}(V([\mathbf{f}, Q]))$, and let $\tilde{\mathbf{x}}=\left(x_{e+1}, \ldots, x_{n}\right)$. Let also $\mathbf{f}_{\mathbf{x}^{\prime}}$ and $\Delta_{\mathbf{x}^{\prime}}^{\prime}$ be the systems in $\mathbf{C}\left[X_{e+1}, \ldots, X_{n}\right]$ obtained by letting $X_{1}=x_{1}, \ldots, X_{e}=x_{e}$ in $\mathbf{f}$ and $\Delta^{\prime}$. Then $\operatorname{jac}_{\tilde{\mathbf{x}}}\left(\left(\mathbf{f}_{\mathbf{x}^{\prime}}, \Delta_{\mathbf{x}^{\prime}}^{\prime}\right)\right.$, $\left.\left[X_{e+1}, \ldots, X_{n}\right]\right)$ has rank $n-e-(i-1)$.

Let notation be as in the last point. Then, we see that the $\operatorname{Jacobian}_{\mathrm{jac}}\left(\left(\mathbf{f}, \Delta^{\prime}, X_{1}-\right.\right.$ $\left.\left.x_{1}, \ldots, X_{e}-x_{e}\right),\left[X_{e+1}, \ldots, X_{n}\right]\right)$ has rank $n-(i-1)$. By point 4 , this implies that the kernel of this Jacobian matrix is the tangent space $T_{\mathbf{x}} W_{i, Q}$. From this observation we deduce that $\mathbf{x}$ belongs to $\operatorname{crit}\left(\Pi_{e, 1}, W_{i, Q}\right)$ if and only if it cancels all $(n-e-i+1)$ minors of $\operatorname{jac}\left(\left(\mathbf{f}, \Delta^{\prime}\right),\left[X_{e+2}, \ldots, X_{n}\right]\right)$ or, equivalently, if it cancels the set $\Delta^{\prime \prime}$. In other words, we have established that

$$
V\left(\left[\left(\mathbf{f}, \Delta^{\prime}, \Delta^{\prime \prime}\right), Q\right]\right)-\operatorname{sing}(V([\mathbf{f}, Q]))=\operatorname{crit}\left(\Pi_{e, 1}, W_{i, Q}\right)-\operatorname{sing}(V([\mathbf{f}, Q])) .
$$

Recall now that $V([(\mathbf{f}, \Delta), Q])=\operatorname{crit}\left(\Pi_{e, 1}, V([\mathbf{f}, Q])\right)$ and that it contains $\operatorname{sing}(V([\mathbf{f}, Q]))$. Adjoining it to the previous equality concludes the proof.

Assumptions $\mathbf{H}$ and $\mathbf{H}_{i}^{\prime}$ imply that $\operatorname{crit}\left(\Pi_{e, 1}, V([\mathbf{f}, Q])\right) \cup \operatorname{crit}\left(\Pi_{e, 1}, W_{i, Q}\right)$ is finite. Thus, calling Solve $((\mathbf{f}, \Delta), Q, 0)$ and $\operatorname{Solve}\left(\left(\mathbf{f}, \Delta^{\prime}, \Delta^{\prime \prime}\right), Q, 0\right)$ gives us two parameterizations $R$ and $R^{\prime}$ whose union $R^{\prime \prime}$ solves our problem. Using Lemmas 25 and 21 and the bounds on the degrees and number of elements in $\Delta, \Delta^{\prime}, \Delta^{\prime \prime}$, we see that $R^{\prime \prime}$ is computed in probabilistic time $\delta_{Q}^{O(1)} n^{O(n \min (p, n-p-e))}(n D) O(n)$ and that $\delta_{R^{\prime \prime}} \leq \delta_{Q}(n D){ }^{O(n)}$.

Computing Fibers (Proof of Lemma 28) Given two zero-dimensional parameterizations $P\left(X_{1}, \ldots, X_{n}\right)$ and $Q\left(X_{1}, \ldots, X_{e}\right)$, we are to compute a parameterization $R$ of $Z(P) \cap \Pi_{e}^{-1}(Z(Q))$. Write $P=\left(p, p_{1}, \ldots, p_{n}\right)$ and $Q=\left(q, q_{1}, \ldots, q_{e}\right)$, and let $\mu=\mu_{1} X_{1}+\cdots+\mu_{e} X_{e}$ be the primitive element of $Q$. We compute $m=$ $\mu_{1} p_{1}+\cdots+\mu_{e} p_{e} \in \mathbf{Q}[T]$ and the polynomials $q(m), p_{1}-q_{1}(m), \ldots, p_{e}-q_{e}(m)$. Then, we replace $p$ by $p^{\prime}=p / \operatorname{gcd}\left(p, q(m), p_{1}-q_{1}(m), \ldots, p_{e}-q_{e}(m)\right)$ and reduce all $p_{i}$ modulo $p^{\prime}$. The cost is polynomial in $n$ and $\delta_{P}+\delta_{Q}$.

Lifting Points on Critical Loci (Proof of Lemma 29) Let $\Delta$ be the set of all $p$-minors of jac $\left(\mathbf{f},\left[X_{e+i+1}, \ldots, X_{n}\right]\right)$. It contains $\left(\begin{array}{c}n-e-i \\ p\end{array}\right) \leq 2^{n}$ polynomials of degree bounded by $n D$. Since $\mathbf{H}$ is satisfied, we have $\operatorname{crit}\left(\Pi_{e, i}, V([\mathbf{f}, Q])\right)=V([\mathbf{f}, \Delta], Q)$; thus, instead of computing $W_{i, Q} \cap \Pi_{e+i-1}^{-1}\left(Z\left(Q^{\prime}\right)\right)$, we compute $\operatorname{crit}\left(\Pi_{e, i}, V([\mathbf{f}, Q])\right) \cap$ $\Pi_{e+i-1}^{-1}\left(Z\left(Q^{\prime}\right)\right)$ : the extra points are all in $\operatorname{sing}(V([\mathbf{f}, Q]))$ and thus in finite number. As a consequence, by Theorem 19.5, $\operatorname{crit}\left(\Pi_{e, i}, V([\mathbf{f}, Q])\right) \cap \Pi_{e+i-1}^{-1}\left(Z\left(Q^{\prime}\right)\right)$ is finite.

Now, we have $\operatorname{crit}\left(\Pi_{e, i}, V([\mathbf{f}, Q])\right)=V([\mathbf{f}, \Delta], Q)=V(\mathbf{f}, \Delta) \cap \Pi_{e}^{-1}(Z(Q))$, so we are to compute

$$
V(\mathbf{f}, \Delta) \cap \Pi_{e}^{-1}(Z(Q)) \cap \Pi_{e+i-1}^{-1}\left(Z\left(Q^{\prime}\right)\right)
$$

This can be rewritten as

$$
V(\mathbf{f}, \Delta) \cap \Pi_{e+i-1}^{-1}(X),
$$


where $X=Z\left(Q^{\prime}\right) \cap \pi^{-1}(Z(Q))$, and $\pi$ is the projection $\mathbf{C}^{e+i-1} \rightarrow \mathbf{C}^{e}$. So, we first compute a parameterization $R$ of $X$, using the function Lift of the previous paragraph, and we return Solve $((\mathbf{f}, \Delta), R, 0)$; the time and degree bounds follow easily from Lemmas 25 and 28.

\section{Proof of the Genericity Properties}

Given a system $\mathbf{f}=\left(f_{1}, \ldots, f_{p}\right)$ and a zero-dimensional parameterization $Q\left(X_{1}, \ldots\right.$, $X_{e}$ ) such that [f, $Q$ ] satisfies $\mathbf{H}$, the algorithms of Sects. 5.4 and 5.5 rely on the fact that assumption $\mathbf{H}_{i}^{\prime}$ holds in generic coordinates for some $i$ and $p$ (Lemmas 31 and 34).

Suppose for the moment that $Q$ is empty and fix $i$ in $\{2, \ldots, n-p\}$. Then, we recall that $\mathbf{f}$ satisfies condition $\mathbf{H}$ if the following holds:

(a) the ideal $\left\langle f_{1}, \ldots, f_{p}\right\rangle$ is radical;

(b) $V=V\left(f_{1}, \ldots, f_{p}\right)$ is equidimensional of positive dimension $d=n-p>0$;

(c) $\operatorname{sing}(V)$ is finite;

(d) $V \cap \mathbf{R}^{n}$ is bounded.

Similarly, $\mathbf{f}$ satisfies $\mathbf{H}_{i}^{\prime}$ if the following holds:

(a) $V=V(\mathbf{f})$ is in Noether position for $\Pi_{d}$;

(b) either $W_{i}$ is empty, or $W_{i}$ is $(i-1)$-equidimensional and in Noether position for $\Pi_{i-1}$

(c) $\operatorname{crit}\left(\Pi_{1}, V\right)$ is finite;

(d) $\operatorname{crit}\left(\Pi_{1}, W_{i}\right)$ is finite;

(e) for $\mathbf{x}$ in $W_{i}-\operatorname{sing}(V), \operatorname{jac}_{\mathbf{x}}\left([\mathbf{f}, \Delta],\left[X_{1}, \ldots, X_{n}\right]\right)$ has rank $n-(i-1)$.

Recall also that we say that [f, $Q]$ satisfies $\mathbf{H}$ if for all $\mathbf{x}$ in $Z(Q)$, the system $\left(f_{1, \mathbf{x}}, \ldots, f_{p, \mathbf{x}}\right)$ satisfies $\mathbf{H}$ in $\mathbf{C}\left[X_{e+1}, \ldots, X_{n}\right]$; similarly, we say that [f, $\left.Q\right]$ satisfies $\mathbf{H}_{i}^{\prime}$ if for all $\mathbf{x}$ in $Z(Q)$, the system $\left(f_{1, \mathbf{x}}, \ldots, f_{p, \mathbf{x}}\right)$ satisfies $\mathbf{H}_{i}^{\prime}$ in $\mathbf{C}\left[X_{e+1}, \ldots, X_{n}\right]$. Thus, in order to prove Lemmas 31 and 34, one can suppose that $Q$ is empty. Lemma 31 discusses $p$ arbitrary, and Lemma 34 has $p=1$.

Proof of Assertion (b) of Lemma 34 Suppose here that $p=1$, and write $f_{1}=f$ and $V=V(f)$. Assuming that $f$ satisfies $\mathbf{H}$, we must prove that $\mathbf{f}=\left[f, \frac{\partial f}{\partial X_{i+1}}, \ldots, \frac{\partial f}{\partial X_{n}}\right]$ satisfies $\mathbf{H}$ in generic coordinates. By [5, Theorem 6], up to a generic linear change of coordinates, $\operatorname{jac}\left(\mathbf{f},\left[X_{1}, \ldots, X_{n}\right]\right)$ has maximal rank at any point $\mathbf{x} \in V(\mathbf{f})-\operatorname{sing}(V)$. Since $V(f)$ is not empty, $\operatorname{sing}(V)$ is finite, and all irreducible components of $V$ have positive dimension, Lemma 15 shows that this implies that $\langle\mathbf{f}\rangle$ is radical, equidimensional of dimension $i-1$. Thus $\mathbf{H}(a)$ and $\mathbf{H}(b)$ are proved for that system. Since the set of points of $V(\mathbf{f})$ at which $\operatorname{jac}\left(\mathbf{f},\left[X_{1}, \ldots, X_{n}\right]\right)$ has not full rank is contained in $\operatorname{sing}(V)$, which is finite by assumption, $\mathbf{H}(c)$ is immediate. Point $\mathbf{H}(d)$ is straightforward since $V(\mathbf{f}) \cap \mathbf{R}^{n} \subset V(f) \cap \mathbf{R}^{n}$, which is bounded.

Proof of $\mathbf{H}_{i}^{\prime}(a)(b)(c)$ and (e) with $1 \leq p \leq n-1$ and $2 \leq i \leq n-p$ in Generic Coordinates In generic coordinates, Corollary 7 in [7] shows that $W_{i}$ is either empty 
or equidimensional of dimension $i-1$ for $i=1, \ldots, n-p$. Assume that it is not empty; then, $\mathbf{H}_{i}^{\prime}(a)$ and $\mathbf{H}_{i}^{\prime}(b)$ are established in [33] when $\operatorname{sing}(V)=\emptyset$. The assumption $\operatorname{sing}(V)=\emptyset$ was only used to ensure that $W_{i}$ had dimension $i-1$, so we obtain $\mathbf{H}_{i}^{\prime}(a)$ and $\mathbf{H}_{i}^{\prime}(b)$ in our case as well. Point $\mathbf{H}_{i}^{\prime}(c)$ says that $\operatorname{crit}\left(\Pi_{1}, V\right)$ is finite; it follows from the first claim with $i=1$, since $\operatorname{crit}\left(\Pi_{1}, V\right)=W_{1} \cup \operatorname{sing}(V)$. Point $\mathbf{H}_{i}^{\prime}(e)$ is in [7, Proposition 8].

Proof of $\mathbf{H}_{2}^{\prime}(d)$ with $1 \leq p \leq n-1$ in Generic Coordinates $\operatorname{By} \mathbf{H}_{2}^{\prime}(b)$, in generic coordinates, $W_{2}$ is a curve in Noether position for $\Pi_{1}$. This easily implies point $\mathbf{H}_{2}^{\prime}(d)$ and thus finishes the proof of Lemma 31.

Proof of $\mathbf{H}_{i}^{\prime}(d)$ with $p=1$ in Generic Coordinates This case turns out to be substantially harder than the other ones. Since we suppose that $p=1$, we write $f_{1}=f$ and $V=V(f)$. We will work with the parameter space $\mathbf{C}^{i} \times \mathbf{C}^{n i}$; to an element $(\mathbf{g}, \mathbf{e})$ of $\mathbf{C}^{i} \times \mathbf{C}^{n i}$ with $\mathbf{e}=\left(\mathbf{e}_{1}, \ldots, \mathbf{e}_{i}\right)$ and all $\mathbf{e}_{k}$ in $\mathbf{C}^{n}$, we associate the linear maps

$$
\begin{aligned}
& \Pi_{\mathbf{e}}: \mathbf{C}^{n} \rightarrow \mathbf{C}^{i}, \\
& \quad \mathbf{x}=\left(x_{1}, \ldots, x_{n}\right) \mapsto\left(\mathbf{e}_{1} \cdot \mathbf{x}, \ldots, \mathbf{e}_{i} \cdot \mathbf{x}\right) \quad \text { and } \\
& \rho_{\mathbf{g}}: \\
& \quad \mathbf{C}^{i} \rightarrow \mathbf{C}, \\
& \quad \mathbf{y}=\left(y_{1}, \ldots, y_{i}\right) \mapsto \mathbf{g} \cdot \mathbf{y} .
\end{aligned}
$$

We also define $W_{\mathbf{e}}$ as the Zariski closure of the set of critical points $\operatorname{crit}\left(\Pi_{\mathbf{e}}, \operatorname{reg}(V)\right)$. First, we need to relate these critical points to the critical points of $\Pi_{i}$ in generic coordinates. If $\mathbf{A}$ is in $\mathrm{GL}_{n}(\mathbf{C})$, we let $f_{\mathbf{A}}$ be the polynomial $f(\mathbf{A X})$, and we let $V_{\mathbf{A}}$ be the zero-set of $f_{\mathbf{A}}$; it is the image of $V$ through the map $\phi: \mathbf{x} \mapsto \mathbf{A}^{-1} \mathbf{x}$. We define the polar variety $W_{i, \mathbf{A}}$ as the polar variety associated to the polynomial $f_{\mathbf{A}}$. Then, the following lemma follows from a straightforward verification.

Lemma 37 Let $\mathbf{A}$ be in $\mathrm{GL}_{n}(\mathbf{C})$, let $\mathbf{e}=\left(\mathbf{e}_{1}, \ldots, \mathbf{e}_{i}\right)$, where $\mathbf{e}_{j}^{t}$ is the jth row of $\mathbf{A}^{-1}$, and let $\mathbf{g}_{0}=\left[\begin{array}{llll}1 & 0 & \cdots & 0\end{array}\right]^{t}$. Then the following equalities hold:

- $W_{i, \mathbf{A}}=\phi\left(W_{\mathbf{e}}\right)$;

- assuming that $W_{i, \mathbf{A}}$ is nonempty and equidimensional, $\operatorname{crit}\left(\Pi_{1}, W_{i, \mathbf{A}}\right)=$ $\phi\left(\operatorname{crit}\left(\rho_{\mathbf{g}_{0}} \circ \Pi_{\mathbf{e}}, W_{\mathbf{e}}\right)\right)$.

In view of this lemma, it is sufficient to prove that for a generic $\mathbf{e}, \operatorname{crit}\left(\rho_{\mathbf{g}_{0}} \circ \Pi_{\mathbf{e}}, W_{\mathbf{e}}\right)$ is finite; along the way, we will also prove that $\phi\left(W_{\mathbf{e}}\right)$ is generically $(i-1)$ equidimensional (if not empty), which reestablishes $\mathbf{H}_{i}^{\prime}(b)$ for hypersurfaces.

First, we give some useful, and well-known, properties of the sets $W_{\mathbf{e}}$. For e in $\mathbf{C}^{n i}$ and $i+1 \leq \ell \leq n$, let $M_{\mathbf{e}, \ell}$ be the $(i+1)$-minor built on columns $(1, \ldots, i, \ell)$ of the $(i+1) \times n$ matrix

$$
\mathbf{M}_{\mathbf{e}}=\left[\begin{array}{c}
\mathbf{e}_{1}^{t} \\
\vdots \\
\mathbf{e}_{i}^{t} \\
\operatorname{grad}(f)
\end{array}\right]
$$

We say that property $\mathbf{a}_{1}(\mathbf{e})$ is satisfied if the following holds: 
- $W_{\mathbf{e}}$ is the zero-set of $\left(f, M_{\mathbf{e}, i+1}, \ldots, M_{\mathbf{e}, n}\right)$,

- the Jacobian matrix of $\left(f, M_{\mathbf{e}, i+1}, \ldots, M_{\mathbf{e}, n}\right)$ has rank $n-i+1$ at all points of $W_{\mathbf{e}}-\operatorname{sing}(V)$,

- $W_{\mathbf{e}}$ is $(i-1)$-equidimensional.

We also need to take into account an alternative property, denoted by $\mathbf{a}_{1}^{\prime}(\mathbf{e})$ :

- $W_{\mathbf{e}}$ is empty.

Our first task is to prove that one of these conditions is generic. Let $\mathbf{E}=\left(\mathbf{E}_{1}, \ldots, \mathbf{E}_{i}\right)$ be $n i$ indeterminates that stand for the entries of the vectors $\mathbf{e}=\left(\mathbf{e}_{1}, \ldots, \mathbf{e}_{i}\right)$. We define the minors $M_{\mathbf{E}, i+1}, \ldots, M_{\mathbf{E}, n}$ as before but leaving $\mathbf{E}$ as indeterminates, and let $K$ be the zero-set of $\left(f, M_{\mathbf{E}, i+1}, \ldots, M_{\mathbf{E}, n}\right)$ in $\mathbf{C}^{n i} \times \mathbf{C}^{n}$.

Lemma 38 Exactly one of the following holds:

1. for a generic $\mathbf{e}$ in $\mathbf{C}^{n i}$, property $\mathbf{a}_{1}(\mathbf{e})$ is satisfied;

2. for a generic $\mathbf{e}$ in $\mathbf{C}^{n i}$, property $\mathbf{a}_{1}^{\prime}(\mathbf{e})$ is satisfied.

Proof The proof of [5, Proposition 3] establishes that for generic e, the following holds:

- $\operatorname{crit}\left(\Pi_{\mathbf{e}}, V\right)$ is the zero-set of $\left(f, M_{\mathbf{e}, i+1}, \ldots, M_{\mathbf{e}, n}\right)$,

- the Jacobian matrix of $\left(f, M_{\mathbf{e}, i+1}, \ldots, M_{\mathbf{e}, n}\right)$ has rank $n-i+1$ at all points of $\operatorname{crit}\left(\Pi_{\mathbf{e}}, V\right) \operatorname{sing}(V)$,

- $\operatorname{crit}\left(\Pi_{\mathbf{e}}, V\right)$ is either empty or $(i-1)$-equidimensional.

Let us first justify that $\operatorname{crit}\left(\Pi_{\mathbf{e}}, V\right)=W_{\mathbf{e}}$. As was the case for $W_{i}$ and $\operatorname{crit}\left(\Pi_{i}, V\right)$, we have now that $\operatorname{crit}\left(\Pi_{\mathbf{e}}, V\right)=W_{\mathbf{e}} \cup \operatorname{sing}(V)$. If $\operatorname{crit}\left(\Pi_{\mathbf{e}}, V\right)$ is empty, our conclusion obviously holds. Else, using the first point and Krull's theorem, we deduce that all irreducible components of $\operatorname{crit}\left(\Pi_{\mathbf{e}}, V\right)$ have positive dimension $i-1 \geq 1$, so there is no isolated point. Since $\operatorname{sing}(V)$ is finite, we deduce that it is included in $W_{\mathbf{e}}$, so that $\operatorname{crit}\left(\Pi_{\mathbf{e}}, V\right)=W_{\mathbf{e}}$.

To conclude, we discuss the dimension of $W_{\mathbf{e}}$ for a generic e. If $K$ is empty, $W_{\mathbf{e}}$ is empty for all e, so $\mathbf{a}_{1}^{\prime}(\mathbf{e})$ holds for all e. Else, let $K_{1}, \ldots, K_{s}$ be the irreducible components of $K$, and let $\mu$ be the projection $\mathbf{C}^{n i} \times \mathbf{C}^{n} \rightarrow \mathbf{C}^{n i}$ on the $\mathbf{E}$-space. If for some $j, \mu\left(K_{j}\right)$ is dense in $\mathbf{C}^{n i}$, then for a generic $\mathbf{e}, W_{\mathbf{e}}$ is not empty; in view of the third point above, this implies that $\mathbf{a}_{1}(\mathbf{e})$ holds. Else, for all $j, \mu\left(K_{j}\right)$ is contained in a hypersurface of $\mathbf{C}^{n i}$; in this case, for a generic $\mathbf{e}, W_{\mathbf{e}}$ is empty, and $\mathbf{a}_{1}^{\prime}(\mathbf{e})$ holds.

Suppose that we are in the second case of the former lemma. Then, for a generic $\mathbf{e}, \operatorname{crit}\left(\rho_{\mathbf{g}_{0}} \circ \Pi_{\mathbf{e}}, W_{\mathbf{e}}\right)$ is a fortiori empty (and thus finite), so we are actually done in this case. Consequently, in all that follows, we assume that we are in the first case of the lemma.

For $0 \leq j \leq i$, define $S_{j}=\left\{\mathbf{x} \in \operatorname{reg}(V) \mid \operatorname{dim}\left(\Pi_{\mathbf{e}}\left(T_{\mathbf{x}} V\right)\right)=j\right\}$. The sets $S_{j}$ form a partition of reg $(V)$; we say that property $\mathbf{a}_{2}(\mathbf{e})$ is satisfied if the following holds:

- for $j=0, \ldots, i, S_{j}$ is either empty or a nonsingular locally closed subset of $\operatorname{reg}(V)$. 
If $\mathbf{a}_{2}(\mathbf{e})$ holds, let $m(n, i, j)=\max \left(0, \operatorname{dim}\left(S_{j}\right)-n+1+j\right)$ and $M(n, i, j)=$ $\operatorname{dim}\left(S_{j}\right)$. Then for $m(n, i, j) \leq \ell \leq M(n, i, j)$, define finally

$$
S_{j, \ell}=\left\{\mathbf{x} \in S_{j} \mid \operatorname{dim}\left(\Pi_{\mathbf{e}}\left(T_{\mathbf{x}} S_{j}\right)\right)=\ell\right\} .
$$

Under $\mathbf{a}_{2}(\mathbf{e})$, the sets $S_{j, \ell}$ form a partition of $S_{j}$. Then, we say that property $\mathbf{a}_{3}(\mathbf{e})$ holds if

- for $j=0, \ldots, i$ and $m(n, i, j) \leq \ell \leq M(n, i, j), S_{j, \ell}$ is either empty or a nonsingular locally closed subset of $S_{j}$.

Then, we can state the following extension of the former lemma.

Lemma 39 For a generic $\mathbf{e}$ in $\mathbf{C}^{n i}$, properties $\mathbf{a}_{2}(\mathbf{e})$ and $\mathbf{a}_{3}(\mathbf{e})$ are satisfied. If $S_{j, \ell}$ is not empty, the inequality $\operatorname{dim}\left(S_{j, \ell}\right) \leq \ell$ holds for $\ell \leq i-1$ and $m(n, i, j) \leq \ell \leq$ $M(n, i, j)$.

Proof Remark that the sets $S_{j}$ and $S_{j, \ell}$ can be rewritten in terms of the standard notation of Thom-Boardman strata [11,37]. Using our notation, Mather's transversality result $[2,3,30]$ shows that for generic $\mathbf{e}, \mathbf{a}_{2}(\mathbf{e})$ and $\mathbf{a}_{3}(\mathbf{e})$ are satisfied, and, if the set $S_{j}$ (resp. $S_{j, \ell}$ ) is not empty, their dimensions are given by

$$
\begin{aligned}
\operatorname{dim}\left(S_{j}\right) & =n-1-v_{n, i}(n-1-j), \\
\operatorname{dim}\left(S_{j, \ell}\right) & =n-1-v_{n, i}\left(n-1-j, \operatorname{dim}\left(S_{j}\right)-\ell\right),
\end{aligned}
$$

where the function $v_{n, i}$ is defined as follows. Considering two indices $r \geq s \geq 0$, we define $\mu(r, s)=r(s+1)-s(s-1) / 2$. Then, we have

$$
\begin{aligned}
v_{n, i}(r) & =(i-n+1+r) r, \\
v_{n, i}(r, s) & =(i-n+1+r) \mu(r, s)-(r-s) s \\
& =(i-n+1+r)\left(r(s+1)-\frac{s(s-1)}{2}\right)-(r-s) s .
\end{aligned}
$$

It remains to check that, under these constraints, we always have $\operatorname{dim}\left(S_{j, \ell}\right) \leq \ell$ for $\ell \leq i-1$; this follows from a straightforward but tedious verification.

Let $\mathbf{G}=\left(G_{1}, \ldots, G_{i}\right)$ be indeterminates for $\mathbf{g}=\left(g_{1}, \ldots, g_{i}\right)$, and let $J$ be the $(n-i+1) \times n$ Jacobian matrix of the polynomials $\left(f, M_{\mathbf{E}, i+1}, \ldots, M_{\mathbf{E}, n}\right)$, where we take partial derivatives in the variables $X_{1}, \ldots, X_{n}$ only. Let further $\mathbf{r}$ be the row vector of length $n$ given by

$$
\mathbf{r}=\left[\begin{array}{lll}
G_{1} & \cdots & G_{i}
\end{array}\right]\left[\begin{array}{c}
\mathbf{E}_{1}^{t} \\
\vdots \\
\mathbf{E}_{i}^{t}
\end{array}\right],
$$

and let $J^{\prime}$ be the $(n-i+2) \times n$ matrix obtained by adjoining the row $\mathbf{r}$ to $J$. We let $X \subset \mathbf{C}^{i} \times \mathbf{C}^{n i} \times \mathbf{C}^{n}$ be the algebraic set defined by $f, M_{\mathbf{E}, i+1}, \ldots, M_{\mathbf{E}, n}$ and all 
$(n-i+2)$-minors of $J^{\prime}$. Finally, we define the projections

$$
\begin{gathered}
\alpha: \mathbf{C}^{i} \times \mathbf{C}^{n i} \times \mathbf{C}^{n} \rightarrow \mathbf{C}^{i} \times \mathbf{C}^{n i}, \quad \text { and } \quad \begin{array}{c}
\gamma: \mathbf{C}^{i} \times \mathbf{C}^{n i} \times \mathbf{C}^{n} \rightarrow \mathbf{C}^{n i} \\
(\mathbf{g}, \mathbf{e}, \mathbf{x}) \mapsto(\mathbf{g}, \mathbf{e})
\end{array}
\end{gathered}
$$

for $\mathbf{e}$ in $\mathbf{C}^{n i}$, we denote by $X_{\mathbf{e}}$ the fiber $X \cap \gamma^{-1}(\mathbf{e})$, and we define

$$
\begin{aligned}
\beta_{\mathbf{e}}: & X_{\mathbf{e}} \rightarrow \mathbf{C}^{n}, \\
& (\mathbf{g}, \mathbf{e}, \mathbf{x}) \mapsto \mathbf{x} .
\end{aligned}
$$

Our goal is now to give an upper bound on the dimension of the fibers $X_{\mathbf{e}}$ (Lemma 42). In the following lemma, we start by estimating in particular the dimension of $\beta_{\mathbf{e}}^{-1}(\mathbf{x})$ for $\mathbf{e}$ and $\mathbf{x}$ fixed; remark that this is an affine space.

Lemma 40 Suppose that $\mathbf{a}_{1}(\mathbf{e})$ holds. For $\mathbf{x}$ in $\operatorname{reg}\left(W_{\mathbf{e}}\right)$ and $\mathbf{g}$ in $\mathbf{C}^{i},(\mathbf{g}, \mathbf{e}, \mathbf{x})$ is in $X_{\mathbf{e}}$ if and only if $\mathbf{x}$ is in $\operatorname{crit}\left(\rho_{\mathbf{g}} \circ \Pi_{\mathbf{e}}, W_{\mathbf{e}}\right)$, and the equality $\operatorname{dim}\left(\Pi_{\mathbf{e}}\left(T_{\mathbf{x}} W_{\mathbf{e}}\right)\right)+$ $\operatorname{dim}\left(\beta_{\mathbf{e}}^{-1}(\mathbf{x})\right)=i$ holds.

Proof Since $\mathbf{a}_{1}(\mathbf{e})$ holds, the polynomials $f, M_{\mathbf{e}, i+1}, \ldots, M_{\mathbf{e}, n}$ define $W_{\mathbf{e}}$, and for $\mathbf{x}$ in $\operatorname{reg}\left(W_{\mathbf{e}}\right)$, the matrix $J$ has rank $n-i+1$ at $\mathbf{x}$. The first claim follows readily, since the last row of $J^{\prime}$ is precisely the vector representing the linear form $\rho_{\mathbf{g}} \circ \Pi_{\mathbf{e}}$. Thus, $(\mathbf{g}, \mathbf{e}, \mathbf{x})$ is in $\beta_{\mathbf{e}}^{-1}(\mathbf{x})$ if and only if for all $\mathbf{v}$ in $T_{\mathbf{x}} W_{\mathbf{e}}, \rho_{\mathbf{g}}\left(\Pi_{\mathbf{e}}(\mathbf{v})\right)=0$ or, equivalently, if for all $\mathbf{w}$ in $\Pi_{\mathbf{e}}\left(T_{\mathbf{x}} W_{\mathbf{e}}\right), \rho_{\mathbf{g}}(\mathbf{w})=0$. Since $\rho_{\mathbf{g}}(\mathbf{w})=\mathbf{g} \cdot \mathbf{w}$, we are done.

Next, we recall a consequence of the theorem on the dimension of fibers.

Lemma 41 If $g$ is polynomial map $A \rightarrow B$ (not necessarily dominant) with $A$ an irreducible algebraic set and $B$ a constructible set and if there exists a fiber of $g$ of dimension $r \geq 0$, then $\operatorname{dim}(A) \leq r+\operatorname{dim}(B)$.

Proof The Zariski closure $C$ of $g(A)$ is contained in an irreducible component of the Zariski closure of $B$, and thus $\operatorname{dim}(C) \leq \operatorname{dim}(B)$. If there exists a fiber of dimension $r$, we get (by the theorem on the dimension of fibers) that $r \geq \operatorname{dim}(A)-\operatorname{dim}(C)$, so $\operatorname{dim}(A) \leq r+\operatorname{dim}(C) \leq r+\operatorname{dim}(B)$, as claimed.

The following lemma gives the key inequality on the dimension of $X_{\mathbf{e}}$.

Lemma 42 Suppose that $\mathbf{a}_{1}(\mathbf{e}), \mathbf{a}_{2}(\mathbf{e})$, and $\mathbf{a}_{3}(\mathbf{e})$ hold. Then $X_{\mathbf{e}}$ has dimension at most $i$.

Proof We fix e that satisfies the assumptions of the lemma. For $0 \leq \ell \leq i-1$, let $j \ell, 1, \ldots, j_{\ell, \kappa(\ell)}$ be the indices $j$ such that $S_{j, \ell}$ is well defined and not empty. Then, we define the constructible sets

$$
T_{\ell}=S_{j \ell, 1}, \ell \cup \cdots \cup S_{j_{\ell, \kappa(\ell)}, \ell} \quad \text { and } \quad T_{\ell}^{\prime}=T_{0} \cup \cdots \cup T_{\ell} .
$$

By Lemma 39, both $T_{\ell}$ and $T_{\ell}^{\prime}$ are disjoint unions of nonsingular locally closed sets of dimension at most $\ell$. Besides, we claim that by Lemma 40, for $0 \leq \ell \leq i$ and for 
$\mathbf{x}$ in $T_{\ell}$, the inequality $\operatorname{dim}\left(\beta_{\mathbf{e}}^{-1}(\mathbf{x})\right) \leq i-\ell$ holds. Indeed, if $\mathbf{x}$ is in $T_{\ell}$, there exists an index $j$ such that $\mathbf{x}$ is in $S_{j, \ell}$, and thus $\operatorname{dim}\left(\Pi_{\mathbf{e}}\left(T_{\mathbf{x}} S_{j}\right)\right)=\ell$. Since $S_{j}$ is contained in $W_{\mathbf{e}}-\operatorname{sing}\left(W_{\mathbf{e}}\right)$, we have $\operatorname{dim}\left(\Pi_{\mathbf{e}}\left(T_{\mathbf{x}} S_{j}\right)\right) \leq \operatorname{dim}\left(\Pi_{\mathbf{e}}\left(T_{\mathbf{x}} W_{\mathbf{e}}\right)\right)$, and we deduce that $\operatorname{dim}\left(\Pi_{\mathbf{e}}\left(T_{\mathbf{x}} W_{\mathbf{e}}\right)\right) \geq \ell$. The bound on $\beta_{\mathbf{e}}^{-1}(\mathbf{x})$ follows from Lemma 40.

Remark that $W_{\mathbf{e}}=T_{i-1}^{\prime} \cup \operatorname{sing}\left(W_{\mathbf{e}}\right)$. Since $T_{i-1}^{\prime}=T_{i-2}^{\prime} \cup T_{i-1}$, we rewrite this as

$$
W_{\mathbf{e}}=T_{i-2}^{\prime} \cup T_{i-1} \cup \operatorname{sing}\left(W_{\mathbf{e}}\right),
$$

where the union is disjoint. Going further, it will be convenient to write, for any $\ell \leq i-1$,

$$
T_{\ell}^{\prime} \cup \operatorname{sing}\left(W_{\mathbf{e}}\right)=T_{\ell-1}^{\prime} \cup T_{\ell} \cup \operatorname{sing}\left(W_{\mathbf{e}}\right) .
$$

Consider now an irreducible component $X^{\prime}$ of $X_{\mathbf{e}}$. By construction, $\beta_{\mathbf{e}}\left(X^{\prime}\right)$ is contained in $W_{\mathbf{e}}$. By (1), either $\beta_{\mathbf{e}}\left(X^{\prime}\right)$ is contained in $T_{i-2}^{\prime} \cup \operatorname{sing}\left(W_{\mathbf{e}}\right)$, or $\beta_{\mathbf{e}}\left(X^{\prime}\right)$ intersects $T_{i-1}$. Suppose first that $\beta_{\mathbf{e}}\left(X^{\prime}\right)$ intersects $T_{i-1}$, so that there exists $(\mathbf{g}, \mathbf{e}, \mathbf{x})$ in $X^{\prime}$ such that $\mathbf{x}$ is in $T_{i-1}$. By the remark in the first paragraph, $\operatorname{dim}\left(\beta_{\mathbf{e}}^{-1}(\mathbf{x})\right) \leq 1$, so that $\operatorname{dim}\left(\beta_{\mathbf{e}}^{-1}(\mathbf{x}) \cap X^{\prime}\right) \leq 1$. In this case, by Lemma $41, \operatorname{dim}\left(X^{\prime}\right) \leq 1+\operatorname{dim}\left(T_{i-1}^{\prime}\right)$, and thus $\operatorname{dim}\left(X^{\prime}\right) \leq i$.

If $\beta_{\mathbf{e}}\left(X^{\prime}\right)$ is contained in $T_{i-2}^{\prime} \cup \operatorname{sing}\left(W_{\mathbf{e}}\right)$, then by (2), either $\beta_{\mathbf{e}}\left(X^{\prime}\right)$ is contained in $T_{i-3}^{\prime} \cup \operatorname{sing}\left(W_{\mathbf{e}}\right)$, or $\beta_{\mathbf{e}}\left(X^{\prime}\right)$ intersects $T_{i-2}$. If $\beta_{\mathbf{e}}\left(X^{\prime}\right)$ intersects $T_{i-2}$, then there is a fiber of dimension at most 2 , so $\operatorname{dim}\left(X^{\prime}\right) \leq 2+\operatorname{dim}\left(T_{i-2}^{\prime}\right) \leq i$. Continuing this way, since $\operatorname{sing}\left(W_{\mathbf{e}}\right)$ is finite, we prove that $\operatorname{dim}\left(X^{\prime}\right) \leq i$ in any case.

Let $\mathcal{F}$ be the Zariski-open subset of $\mathbf{C}^{n i}$ underlying Lemmas 38 and 39: for $\mathbf{e}$ in $\mathcal{F}, \mathbf{a}_{1}(\mathbf{e}), \mathbf{a}_{2}(\mathbf{e})$, and $\mathbf{a}_{3}(\mathbf{e})$ hold. Let then $Z=\mathbf{C}^{n i}-\mathcal{F}$; this is a strict algebraic subset of $\mathbf{C}^{n i}$. On the other hand, let $Y \subset \mathbf{C}^{i} \times \mathbf{C}^{n i}$ be the Zariski closure of the set of all $(\mathbf{g}, \mathbf{e}) \in \mathbf{C}^{i} \times \mathbf{C}^{n i}$ such that the fiber $X \cap \alpha^{-1}(\mathbf{g}, \mathbf{e})$ is infinite.

Lemma 43 The set $Y$ is a strict algebraic subset of $\mathbf{C}^{i} \times \mathbf{C}^{n i}$.

Proof First, $Y$ is obviously Zariski-closed. We continue by proving that it does not cover all of $\mathbf{C}^{i} \times \mathbf{C}^{n i}$. Let $X^{\prime}$ be an irreducible component of $X$.

- If $\gamma\left(X^{\prime}\right)$ does not intersect $\mathcal{F}$, then it is contained in $Z$, which implies that $\alpha\left(X^{\prime}\right)$ as a whole is contained in $\mathbf{C}^{i} \times Z$.

- If $\gamma\left(X^{\prime}\right)$ intersects $\mathcal{F}$, there exists $(\mathbf{g}, \mathbf{e}, \mathbf{x})$ in $X^{\prime}$ such that $\mathbf{e}$ is in $\mathcal{F}$. Then, Lemma 42 implies that $\operatorname{dim}\left(X_{\mathbf{e}}\right) \leq i$, and the theorem on the dimension of fibers implies that $\operatorname{dim}\left(X^{\prime}\right) \leq i+n i$. As a consequence, the set of infinite fibers of the restriction of $\alpha$ to $X^{\prime}$ is contained in a hypersurface of $\mathbf{C}^{i} \times \mathbf{C}^{n i}$.

This finishes the proof that $Y$ is a strict Zariski-closed subset of $\mathbf{C}^{i} \times \mathbf{C}^{n i}$.

Recall that $\mathbf{g}_{0}$ is the vector of length $i$ given by $\left[\begin{array}{llll}1 & 0 & \cdots & 0\end{array}\right]^{t}$, and let $Y^{\prime} \subset \mathbf{C}^{n i}$ be the set $\left\{\mathbf{e} \in \mathbf{C}^{n i} \mid\left(\mathbf{g}_{0}, \mathbf{e}\right) \in Y\right\}$. Since $Y$ is Zariski-closed, $Y^{\prime}$ is a Zariski-closed subset of $\mathbf{C}^{n i}$. The next lemma refines this observation.

Lemma $44 Y^{\prime}$ is a strict algebraic subset of $\mathbf{C}^{n i}$. 
Proof For any invertible $i \times i$ matrix $\mathbf{M}$, the defining equations of $X$ are multiplied by a nonzero constant through the change of variables $(\mathbf{G}, \mathbf{E}, \mathbf{X}) \mapsto\left(\mathbf{M}^{-1} \mathbf{G}, \mathbf{M E}, \mathbf{X}\right)$, so $X$ is stabilized by this action. Thus, a point $(\mathbf{g}, \mathbf{e})$ in $\mathbf{C}^{i} \times \mathbf{C}^{n i}$ belongs to $Y$ if and only if $\left(\mathbf{M}^{-1} \mathbf{g}, \mathbf{M e}\right)$ does.

Because $Y$ is contained in a hypersurface of $\mathbf{C}^{i} \times \mathbf{C}^{n i}$, there exist $(\tilde{\mathbf{g}}, \tilde{\mathbf{e}})$ in $\mathbf{C}^{i} \times \mathbf{C}^{n i}$ and an open Euclidean neighborhood $B$ of it such that $B \cap Y$ is empty. Let $\mathbf{M}$ be such that $\mathbf{M}^{-1} \tilde{\mathbf{g}}=\mathbf{g}_{0}$, and let $\psi: \mathbf{C}^{i} \times \mathbf{C}^{n i} \rightarrow \mathbf{C}^{i} \times \mathbf{C}^{n i}$ be the map $(\mathbf{g}, \mathbf{e}) \mapsto\left(\mathbf{M}^{-1} \mathbf{g}, \mathbf{M e}\right)$. The image $\psi(B)$ is an open neighborhood of $\left(\mathbf{g}_{0}, \mathbf{e}_{0}\right)$ with $\mathbf{e}_{0}=\mathbf{M} \tilde{\mathbf{e}}$. By the remarks of the previous paragraph, there is no point of $Y$ in $\psi(B)$. This is enough to conclude.

We can now define $\Delta=\mathbf{C}^{n i}-\left(Z \cup Y^{\prime}\right)$, where $Z$ was defined prior to Lemma 43; $\Delta$ is thus a nonempty Zariski-open subset of $\mathbf{C}^{n i}$. The following lemma finishes to establish our main claim.

Lemma 45 For $\mathbf{e}$ in $\Delta$, the set $\operatorname{crit}\left(\rho_{\mathbf{g}_{0}} \circ \Pi_{\mathbf{e}}, W_{\mathbf{e}}\right)$ is finite.

Proof Remark that $\left(\mathbf{g}_{0}, \mathbf{e}\right)$ is in $\mathbf{C}^{i} \times \mathbf{C}^{n i}-Y$. By the definition of $Y$, this implies that $\alpha^{-1}\left(\mathbf{g}_{0}, \mathbf{e}\right)$ intersects $X$ in a finite number of points. Besides, $\mathbf{e}$ is in $\mathcal{F}$, so $\mathbf{a}_{1}(\mathbf{e})$ holds, and we deduce that $\operatorname{sing}\left(W_{\mathbf{e}}\right)$ is finite. For $\mathbf{x}$ in $\operatorname{reg}\left(W_{\mathbf{e}}\right)$, by Lemma $40, \mathbf{x}$ is in $\operatorname{crit}\left(\rho_{\mathbf{g}_{0}} \circ \Pi_{\mathbf{e}}, W_{\mathbf{e}}\right)$ if and only if $\left(\mathbf{g}_{0}, \mathbf{e}, \mathbf{x}\right)$ is in $X_{\mathbf{e}}$ and thus if and only if $\left(\mathbf{g}_{0}, \mathbf{e}, \mathbf{x}\right)$ is in $X \cap \gamma^{-1}\left(\mathbf{g}_{0}, \mathbf{e}\right)$. Since this set is finite, we are done.

Acknowledgements We wish to thank S. Basu, M. Coste, and M.-F. Roy for useful comments and discussions. We acknowledge financial support from NSERC, the Canada Research Chair program, and INRIA.

\section{References}

1. Alonso, M.E., Becker, E., Roy, M.-F., Wörmann, T.: Zeroes, multiplicities and idempotents for zerodimensional systems. In: Algorithms in Algebraic Geometry and Applications. Proceedings of MEGA'94. Progress in Mathematics, vol. 142, pp. 1-15. Birkhaüser, Basel (1996)

2. Alzati, A., Ballico, E., Ottaviani, G.: The theorem of Mather on generic projections for singular varieties. Geom. Dedic. 85(1-3), 113-117 (2001)

3. Alzati, A., Ottaviani, G.: The theorem of Mather on generic projections in the setting of algebraic geometry. Manuscr. Math. 74(4), 391-412 (1992)

4. Atiyah, M., MacDonald, I.: Introduction to Commutative Algebra. Addison-Wesley Series in Mathematics. Addison-Wesley, Reading (1969)

5. Bank, B., Giusti, M., Heintz, J., Mbakop, G.-M.: Polar varieties and efficient real equation solving: the hypersurface case. J. Complex. 13(1), 5-27 (1997)

6. Bank, B., Giusti, M., Heintz, J., Mbakop, G.-M.: Polar varieties and efficient real elimination. Math. Z. 238(1), 115-144 (2001)

7. Bank, B., Giusti, M., Heintz, J., Safey El Din, M., Schost, É.: On the geometry of polar varieties. Appl. Algebra Eng. Commun. Comput. 21(1), 33-83 (2010)

8. Basu, S., Pollack, R., Roy, M.-F.: Computing roadmaps of semi-algebraic sets (extended abstract). In: STOC, pp. 168-173. ACM, New York (1996)

9. Basu, S., Pollack, R., Roy, M.-F.: Computing roadmaps of semi-algebraic sets on a variety. J. AMS 3(1), 55-82 (1999)

10. Basu, S., Pollack, R., Roy, M.-F.: Algorithms in Real Algebraic Geometry, 2nd edn. Algorithms and Computation in Mathematics, vol. 10. Springer, Berlin (2006) 
11. Boardman, J.M.: Singularities of differentiable maps. Publ. Math. Inst. Hautes Études Sci. 33, 21-57 (1967)

12. Bochnak, J., Coste, M., Roy, M.-F.: Real Algebraic Geometry. Ergebnisse der Mathematik und ihrer Grenzgebiete, vol. 36. Springer, Berlin (1998)

13. Brasselet, J.-P., Damon, J., Trang, L.D., Oka, M. (eds.): Singularities in Geometry and Topology. World Scientific, Singapore (2007)

14. Canny, J.: The complexity of robot motion planning. Ph.D. Thesis, MIT (1987)

15. Canny, J.: Computing roadmaps in general semi-algebraic sets. Comput. J. 36(5), 504-514 (1993)

16. Coste, M., Shiota, M.: Nash triviality in families of Nash manifolds. Invent. Math. 108(1), 349-368 (1992)

17. Della Dora, J., Discrescenzo, C., Duval, D.: About a new method for computing in algebraic number fields. In: EUROCAL 85 Vol. 2. LNCS, vol. 204, pp. 289-290. Springer, Berlin (1985)

18. Duval, D.: Rational Puiseux expansions. Compos. Math. 70(2), 119-154 (1989)

19. Eisenbud, D.: Commutative Algebra with a View Toward Algebraic Geometry. Graduate Texts in Mathematics, vol. 150. Springer, Berlin (1995)

20. Faugère, J.-C., Gianni, P., Lazard, D., Mora, T.: Efficient computation of zero-dimensional Gröbner bases by change of ordering. J. Symb. Comput. 16(4), 329-344 (1993)

21. Giusti, M., Heintz, J.: La détermination des points isolés et de la dimension d'une variété algébrique peut se faire en temps polynomial. In: Computational Algebraic Geometry and Commutative Algebra. Symposia Matematica, vol. XXXIV, pp. 216-256. Cambridge University Press, Cambridge (1993)

22. Giusti, M., Heintz, J., Morais, J.-E., Morgenstern, J., Pardo, L.-M.: Straight-line programs in geometric elimination theory. J. Pure Appl. Algebra 124, 101-146 (1998)

23. Giusti, M., Heintz, J., Morais, J.-E., Pardo, L.-M.: When polynomial equation systems can be solved fast? In: AAECC-11. LNCS, vol. 948, pp. 205-231. Springer, Berlin (1995)

24. Giusti, M., Heintz, J., Morais, J.-E., Pardo, L.-M.: Le rôle des structures de données dans les problèmes d'élimination. C. R. Acad. Paris 325, 1223-1228 (1997)

25. Giusti, M., Lecerf, G., Salvy, B.: A Gröbner-free alternative for polynomial system solving. J. Complex. 17(1), 154-211 (2001)

26. Gournay, L., Risler, J.-J.: Construction of roadmaps in semi-algebraic sets. Appl. Algebra Eng. Commun. Comput. 4(4), 239-252 (1993)

27. Heintz, J., Roy, M.-F., Solernó, P.: Single exponential path finding in semi-algebraic sets II: The general case. In: Algebraic Geometry and Its Applications, Collections of Papers from Abhyankar's 60-th Birthday Conference. Purdue University, West-Lafayette (1994)

28. Heintz, J., Schnorr, C.P.: Testing polynomials which are easy to compute (extended abstract). In: STOC, pp. 262-272. ACM, New York (1980)

29. Lecerf, G.: Computing an equidimensional decomposition of an algebraic variety by means of geometric resolutions. In: ISSAC'00, pp. 209-216. ACM, New York (2000)

30. Mather, J.N.: Generic projections. Ann. Math. 98, 226-245 (1973)

31. Mumford, D.: Algebraic Geometry I, Complex Projective Varieties. Classics in Mathematics. Springer, Berlin (1976)

32. Rouillier, F.: Solving zero-dimensional systems through the Rational Univariate Representation. Appl. Algebra Eng. Commun. Comput. 9(5), 433-461 (1999)

33. Safey el Din, M., Schost, É.: Polar varieties and computation of one point in each connected component of a smooth real algebraic set. In: ISSAC'03, pp. 224-231. ACM, New York (2003)

34. Schost, É.: Computing parametric geometric resolutions. Appl. Algebra Eng. Commun. Comput. 13(5), 349-393 (2003)

35. Schwartz, J.T., Sharir, M.: On the "piano movers" problem. II. General techniques for computing topological properties of real algebraic manifolds. Adv. Appl. Math. 4(3), 298-351 (1983)

36. Shafarevich, I.: Basic Algebraic Geometry 1. Springer, Berlin (1977)

37. Thom, R.: Les singularités des applications différentiables. Ann. Inst. Fourier 6, 43-87 (1955-1956)

38. Todd, J.A.: The arithmetical invariants of algebraic loci. Proc. Lond. Math. Soc. 43, 190-225 (1937)

39. Zariski, O., Samuel, P.: Commutative Algebra. Van Nostrand, Princeton (1958) 